$$
000 \ldots 156 q-2 q
$$

\title{
A NUCLEAR SPIN ECHO STUDY OF $\mathrm{Mn}_{2-\mathrm{x}} \mathrm{Cr}_{\mathrm{x}} \mathrm{Sb}$
}

W. Weyhmann and R. W. Houghton

\section{Solid State and Low Temperature Physics Group}

SCHOOL OF PHYSICS AND ASTRONOMY

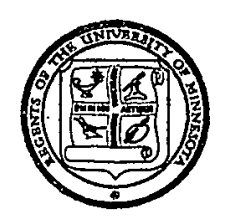

September 1968

UNIVERSITY OF MINNESOTA

MINNEAPOLIS, MINNESOTA

Work supported in part by the U.S. Atomic Energy Commission 


\section{DISCLAIMER}

This report was prepared as an account of work sponsored by an agency of the United States Government. Neither the United States Government nor any agency Thereof, nor any of their employees, makes any warranty, express or implied, or assumes any legal liability or responsibility for the accuracy, completeness, or usefulness of any information, apparatus, product, or process disclosed, or represents that its use would not infringe privately owned rights. Reference herein to any specific commercial product, process, or service by trade name, trademark, manufacturer, or otherwise does not necessarily constitute or imply its endorsement, recommendation, or favoring by the United States Government or any agency thereof. The views and opinions of authors expressed herein do not necessarily state or reflect those of the United States Government or any agency thereof. 


\section{DISCLAIMER}

Portions of this document may be illegible in electronic image products. Images are produced from the best available original document. 
$000-1569-29$

A NUCLEAR SPIN .EOHO

STUDY OF $\mathrm{Mn}_{2 \rightarrow \mathrm{x}} \mathrm{Cr}_{\mathrm{x}} \mathrm{Sb}$ *

W. Weyhmann and R. W. Houghton

Tate Laboratory of Physics

University of Minnesota, Minneapolis, Minnesota

* Work supported in part by the U. S. Atomic

Energy Commission under Contract No. AT $(11-1)$ 1569.

\section{IEGAL NOTICE}

Thls report was prepared as an account of Government sponsored work. Nelther the United States, nor the Commission, nor any person acting on behalf of the Commission:

A. Makes any warranty or representation, expressed or implied, with respect to the accuracy, completeness, or usefuiness of the information contained in this report, or that the use of any information, apparatus, method, or process disclosed in this report may not infringe privately owned rights; or

B. Assumes any liabilitles with respect to the use of, or for damages resulting from the use of any information, apparatus, method, or process disclosed in thls report.

As used in the above, "person acting on behalf of the Commisalon" includes any em plor the ployee or contractor of the corson acting on bebir or the such employee or contractor or the Com, or ampares. disseminates, or provides access to, any fnformation pursuant to his employment or contract with the Commission, or his employment with such contractor. 


\begin{abstract}
ABSTRAOT
We have made nuclear spin echo measurements on $\mathrm{Mn}_{2-\mathrm{x}} \mathrm{Cr} \mathrm{x}_{\mathrm{Sb}}$ from $4^{\circ}$ to $300^{\circ} \mathrm{K}$. Two samples were used: 325 mesh powder and bulk single crystals with chromium concentrations $\mathrm{x}=0$ and $\mathrm{x}=0.03$, respectively. The NMR frequency and nuclear relaxation time of the two inequivalent manganese sites were studied in detali. The NMR frequency data is compared with spin wave theory and calculations of the sublattice magnetization. The crystaline field quadrupole splitting was measmred in the region of the reorientation of the easy axis of magnetization. Although the exact nature of the reorientation could not be determined, a fluctuation in the spin system over a wide temperature interval was observed. These results are compared with recent work by $\mathrm{H}$. Horner and $\mathrm{C}$. Varme, which suggests the presence of two second-order phase transitions. An unsuccessful attempt was made to verify this by observing the specific heat anomalies.
\end{abstract}




\section{ABSTRACT}

We have made nuclear spin echo measurements on $\mathrm{Mn}_{2-\mathrm{x}} \mathrm{Cr} \mathrm{Sb}$ from $4^{\circ}$ to $300^{\circ} \mathrm{K}$. Two samples were used: 325 mesh powder and bulk single crystals with chromium concentrations $x=0$ and $x=0.03$; respectively. The NMR frequency and nuclear relaxation time of the two inequivalent manganese sites were studied in detall. The NMR frequency data is compared with spin wave theory and calculations of the sublatice magnetization. The crystalline field quadrupole splitting was measured in the region of the reorientation of the easy axis of magnetization. Although the exact nature of the reorientation could not be determined, a fluctuation in the spin system over a wide temperature interval was observed. These results are compared with recent work by H. Horner and C. Varma, which suggests the presence of two second-order phase transitions. An unsuccessful attempt was made to verify this by observing the specific heat anomalies. 


\section{ACKNOWLEDGEMENTS}

It is impossible to mention everyone who has assisted in this work. We are particularly grateful to Professor Nosanow, Professor Horner, and Dr. Varma for their stimulating discussions and interest in this research, to Professor Moldover for his collaboration in performing the specific heat measurements, to Mr. Robert Howard and Mr. Gerald Anderson for their help in designing the electrical circuits, to D. Gerard for his help on the magnetization calculations, and to the many graduate students who were always willing to give assistance.

We are also grateful to Mrs. Noreen Lefevre for her help in analyzing some of the data and for her talent and patience in transforming unintelligible manuscript into the final typed copy. The generous assistance of Mrs. Zella Berge for some of the typing is also appreciated.

We are grateful for the generous support of the Graduate school during the early part of this research.

One of us, RWH, is especially grateful to his wife, Beverly, for her patience and support throughout. his graduate study-and for her critical reading of the thesis. He is also grateful to the office of Education for the tenure of a NDEA Title IV fellowship during part of his graduate study. 


\section{ABSTRACT}

ACKNOWLEDGEMENTS

Chapter

Page

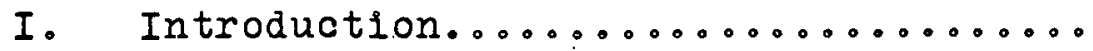

II. General Theory...............

Magnetic structure of $\mathrm{Mn}_{2-\mathrm{x}} \mathrm{Cr}_{\mathrm{x}} \mathrm{Sb} \ldots$

Sublattice Magnetization..........

Magnetic Domain Structure........

Hyperfine Interactions.........

Nuclear Magnet1c Resonance in Ferromagnetic Metals............

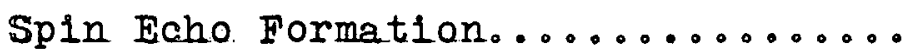

Quadrupole Modulation of Spin. Echoes

NMR Frequency and Local Magnetization

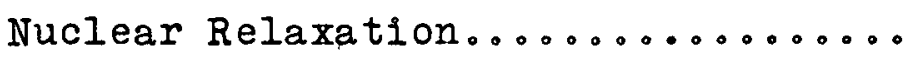

III. Experimental Design and Procedure....

Materials.

Magnetometer................... 47

Spin Echo Spectrometer Design......

Experimental Procedure...........

Relaxation Time Measurements.......

IV. Experimental Results............ 


\section{TABIE OF CONTENTS}

NMR Frequency Measurements.......

Frequency Identification of the

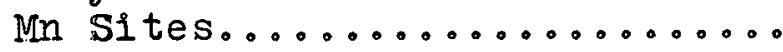

70

Direction of the Hyperfine Field....

Sublattice Magnetization.

Nuclear Relaxation Times $\mathrm{T}_{1}, \mathrm{~T}_{2} \ldots .$.

V. Magnetization Reorientation.........

Introduction.

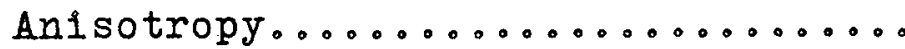

Theory

Experimental Measurements.........

Experimental Results.............

Relationship of Experimental Results to the Horner-Varma Theory.....

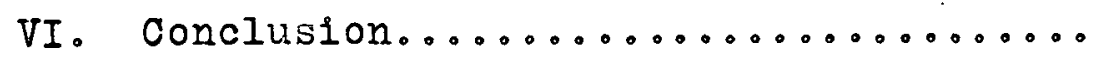

Appendix

A. Foner Magnetometer Design........ A-1

B. Oscilloscope Trace Blanking........ B-1

c. Magnetic Field Pulses............. c-1

D. Specific Heat Apparatus.......... D

References.................................... $R-1$ 
1. Magnetic structure of $\mathrm{Mn}_{2} \mathrm{Sb} \ldots \ldots \ldots \ldots \ldots$

2. Saturation Magnetization of $\mathrm{Mn}_{2}-\mathrm{x} \mathrm{Cr}_{\mathrm{x}} \mathrm{Sb}$.. 7

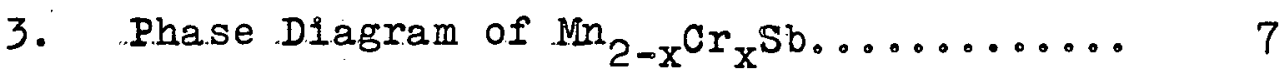

4. .Spin Echo Spectrometer............... 49

5. RF Oscillator and Grid Pulse Amplifier.. 50

6. Coaxial Inine.................... 55

7. Magnet1c Field Dependence of the NMR

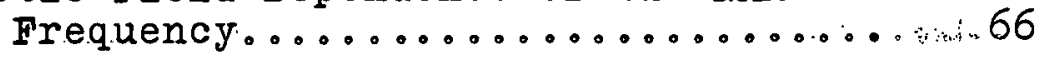

8. RF Power Dependence of $\mathrm{T}_{1}$ and $\mathrm{T}_{2} \ldots \ldots \ldots 66$

9. Magnetic Field Dependence of the Spin Echo Amplitude................ 66

10. Temperature Dependence of the NMR Frequency for Different Nuclear Sites in $\mathrm{Mn}_{2} \mathrm{Sb} \ldots \ldots \ldots \ldots . \ldots . \ldots . \ldots 6$

11. NMR Frequency as a Function of $: \mathrm{T}^{3 / 2} \ldots . .73$

12. Sublattice Magnetization.............. . 74

13. Temperature Dependence of $\mathrm{T}_{1}$ and $\mathrm{T}_{2} \ldots \ldots 76$

14. Temperature Dependence of the Anisotropy Constants....................... 79

15. Anisotropy Free Energy in the Reorientaton

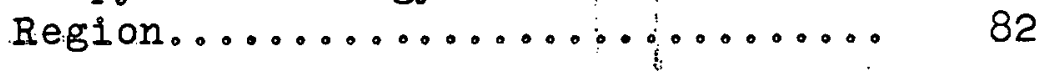

16. Predicted Behavior of Magnetization Orientation and Quadrupole Splitting in the Reorientation Region........

17. Temperature Dependence of the Quadrupole Splitting and the Spin Echo Modulation Amplitude............ 88

18. Photographs of the Spin Echo Envelope... 88

19. Temperature Dependence of $T_{2}$ Near $T_{1} \ldots 91$

20. Temperature Dependence of the NMR

Frequency Near $\mathrm{T}_{\mathrm{r}} \ldots \ldots \ldots \ldots \ldots \ldots$ 


\section{Chapter I}

\section{Introduction}

For many years nuclear magnetic resonance (NMR) has been a standard technique in solid state research. ${ }^{1}$ In contrast, NMR in ferromagnetic magnetic materials is a recent development.and is still not thoroughly understood. NoKurti's successful nuclear orientation of $00^{60}$ in a co crystal demonstrated the existence of strong nuclear hyperfine coupling in a ferromagnet. 2 Stimulated by this work, NMR in Co was first observed by Gossard and Portis in 1959.3

Several properties of ferromagnetic materials introduce new and complicating features to the NMR signals. The strong coupling between nuclei in an ordered magnetic state produces very short relaxation times. Even in the absence of an external magnetic field, the nuclei see very large internal fields due to the hyperfine coupling with the electron magnetization. The effective magnetic fièld seen by the nuclei in the presence of an applied external' field is complicated by domain structure and size effects of the ferromagnetic material. The electron magnetization follows the applied radio frequency ( $f f$ ) fleld so that the NMR, excited via the hyperfine coupling to the electron 
system, is greatly enhanced.

our work is a general NMR study of $\mathrm{Mn}_{2-\mathrm{x}}$ or $\mathbf{S b}$. This material has a complex phase diagram with first-order. magnetic transitions among its ferrimagnetic, antiferromagnetic, and spiral ordered phases. In addition, there is a rotation of the easy axis of magnetization at a temperature $T_{r}$ in the ferrimagnetic and ferromagnetic phases. Our initial intention was to study the NMR frequency across these transitions to obtain information about the hyperfine interactions. In the course of the work. the emphasis was changed to study in detail the reorientation of the axis of magnetization. The majority of the NMR measurements were made using a spin echo spectrometer. Measurements of the macroscopic magnetization were made on a vibrating Foner magnetometer. Finally, a simple specific heat measurement was performed in an unsuccessful attempt to verify a proposed phase transition at the magnetization reorientation.

Our most important result was the possible discovery of some sort of spatial fluctuation in the magnetization at $T_{r}$. This may be related to the broadband. microwave absorption at $T_{r}$ in orthoferrites recently reported by R. C. Lecraw et al. 4 An analysis of this reorientation using a Landau-type description by H. Horner and C. M. Varma ${ }^{5}$ suggests that both of these 
phenomena may be explained as a second-order phase transition. In any case, a complex dynamic model is required to describe the reorientation process.

There is a number of other results from our NINR measurements on $\mathrm{Mn}_{2-\mathrm{x}} \mathrm{Cr}_{\mathrm{x}} \mathrm{Sb}$ over a temperature range of $4^{\circ} \mathrm{K}$ to $273^{\circ} \mathrm{K}$ which are of interest. These are:

1. the magnitude and sign of the hyperfine field,

2. the identification of the resonance frequencies of the two different.Mn sites,

3. the temperature dependence of the $N M R$ frequency and its comparison with the sublattice magnetization and spin wave theory,

4. the temperature dependence of the transverse and longitudinal relaxation times $T_{1}$ and $\mathbb{T}_{2}$, and

5. the temperature dependence of the quadrupole splitting of the zeeman levels.

NINR frequency and relaxation measurements provide information about the nuclear hyperfine coupling in ordered magnetic systems. It is a sensitive measure of the local average magnetic fleld at a nuclear site and-can be used to study the excltation spectrum of the magnetic system. The present theory of ferromagnetic metals is unable to account for the strong coupling that is observed: or even to determine what mechanism dominates. Systematic data of many magnetically 
ordered alloys are needed to arrive at a satisfactory understanding of these interactions.

This work will be organized in the following way. We will begin with a discussion of the magnetic structure and the hyperfine interaction theory, which are necessary for understanding the NMR measurements in magnetically ordered materials. This will be followed by a brief discussion of. NNR theory with emphasis on spin echoes. Next will come the experimental chapter in which we consider sample preparation, description of the apparatus, and experimental procedure. our results and analysis follow in a separate chapter. The final chapter. will be devoted to the reorientation of the easy axls of magnetization. I.t will begin with an outline of the Horner-Varma theory and conclude with our experimental results and analysis. This will include a description of the specific heat measurement and suggestions for further work on this problem. Details of the electrical circuits and the apparatus are described in the appendix. 
Chapter II

General Theory

Magnet1c Structure of $\mathrm{Mn} 2-\frac{\mathrm{Or}}{\mathrm{Sb}}$

...Manganese antimonide, $\mathrm{Mn}_{2} \mathrm{Sb}$, is an intermetallic compound with two molecules per unit cell in a tetragonal crystal structure. It was first studied by Guillaud in 1.943.6 To account. for the saturation magnetization measurements, he proposed the ferrimagnetic structure shown in Fig. 1 in which the Mn atoms occupy two inequivalent sites, $M n(I)$ and $M n(I I)$, magnetized in opposite directions. He also determined the Curie temperature $T_{C}=550^{\circ} \mathrm{K}$ and a rotation of the easy axis of magnetization at $\mathrm{T}_{\mathrm{r}}=240^{\circ} \mathrm{K}$, now known to be $247^{\circ} \mathrm{K}$. The direction of magnetization is parallel to the c-axis for $T_{r}<T<T_{c}$ and perpendicular to the c-axis for $T<T_{r}$.

More recent neutron diffraction measurements by Wilkinson et al. ${ }^{7}$ in 1957 and Alperin et al. ${ }^{8}$ in 1963 confirm this ferrimagnetic structure and show that the $\mathrm{Sb}$ atoms have no magnetic moment. X-ray measurements at room temperature give the lattice constants of the unit cell $\underline{c}=6.56 \AA$ and $\underline{a}=4.08 \AA$. The Mn atoms occupy positions with coordinates $(0,0,0) ;\left(\frac{1}{2}, \frac{1}{2}, 0\right) ;\left(0, \frac{1}{2}, z_{1}\right)$; $\left(\frac{1}{2}, Q_{1} z_{1}\right)$; and the Sb occupy positions $\left(0, \frac{1}{2}, z_{2}\right) ;\left(\frac{1}{2}, 0, z_{2}\right)$ 


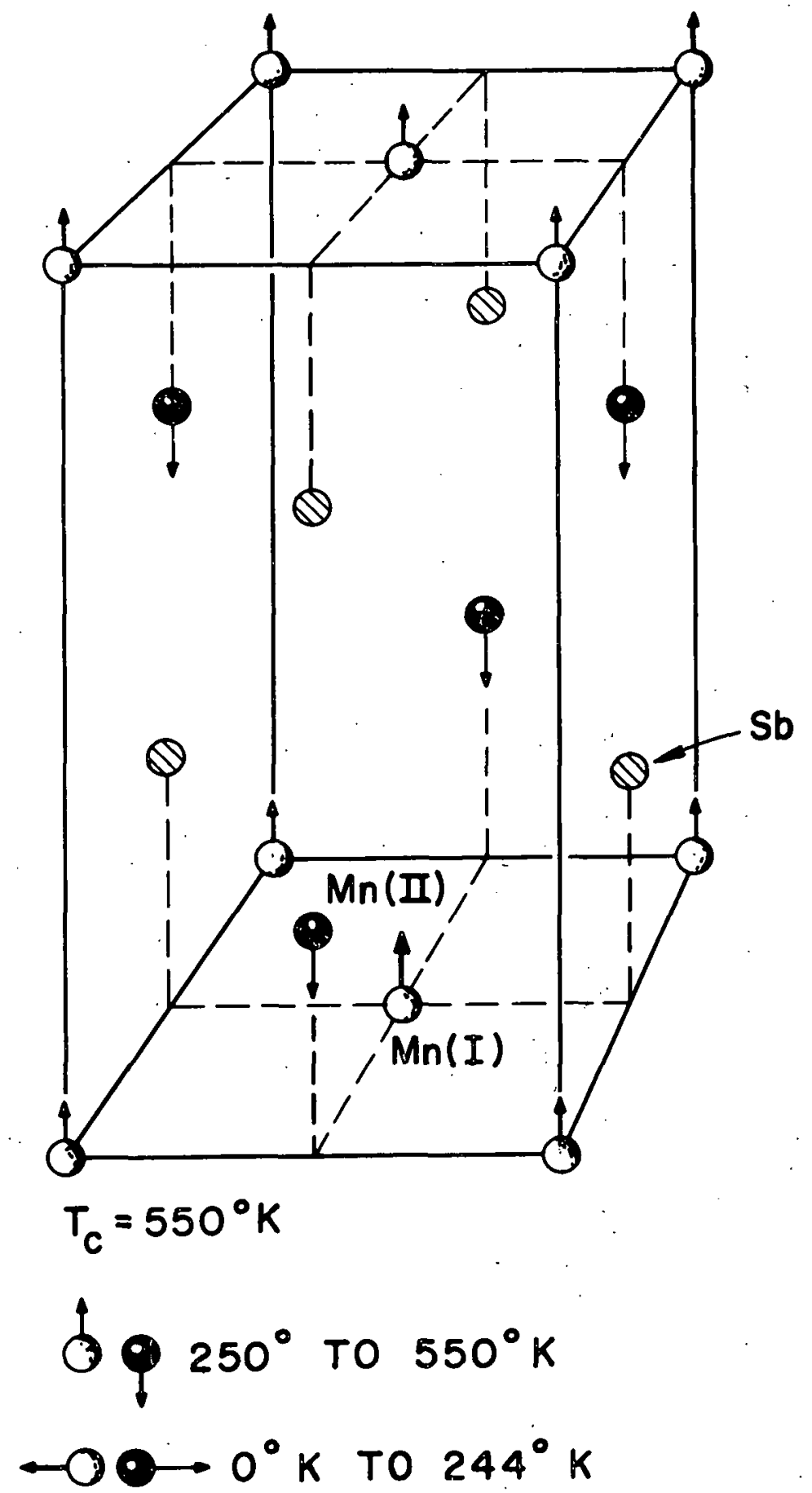

FIGURE 1. MAGTETIO STRUCTURT OF M $\mathrm{N}_{2}$ Sb 
where $z_{1}=0.2897$ and $z_{2}=-0.2793$. The magnetization at $0^{0} \mathrm{~K}$ for the two Mn sites is $\mu_{I}=-1.77 \pm 0.18 \mu_{\beta}$ and $\mu_{I I}=3.55 \pm 0.18 \mu_{\beta}$, where the negative sign indicates magnetization in the opposite direction to the net magnetization of the crystal and $\mu_{\beta}$ is the Bohr magneton. These values are fitted to agree with net magnetization $\mu_{I I}-\mu_{I}$ as obtained from magnetic measurements.

A contour map of the unpaired spin density in $\mathrm{Mn}_{2} \mathrm{Sb}$ by Alperin et al. ${ }^{8}$ reveals a complex structure. The spin density distribution about each Mn site is aspherical and compact. The distribution about the Mn(II) site is displaced slightly toward the nearest Sb atom suggesting that the ferromagnetic ordering among the Mn(II) atoms takes place via these $\mathrm{Sb}$ atoms. It is also found that 5\% of the spin density is nonlocally distributed in the space between atoms, accounting for errors in earlier measurements of the magnetic moments. Finally, it is of interest to understand the reason for the difference in the magnetic moment of each site. The spin distribution is more compact at the $M n$ (I) site than at the Mn(II) site, but both are considerably more compact than the calculated $\mathrm{Mn}^{2+}$ ion radial distribution. 9 If we assumed an essentially lonic bond, the formal charge distribution would be $\operatorname{Mn}(I)^{+} \operatorname{Mn}(I I)^{2+} \cdot \mathrm{S}^{3-} \cdot 10$ However, according to Alperin et al. 8 if the difference in moments were caused by 
differing valency state, one would expect the radial spin distributions of the two sites to differ more than 1s observed. We will be interested in the covalent character of the bonding in later discusisions.

Extensive research has been done on chromium doped $\mathrm{Mn}_{2} \mathrm{Sb}$ at E. I. duPont de Nemours and Company since 19.60..$^{11-13}$. The magnetic sublattice structure of $\mathrm{Mn}_{2} \mathrm{Sb}$ consists of planes perpendicular to the c-axis varying in sequence

$$
\text { - I - II } a-I I_{b}-I-I I_{a}-I I_{b}-I-
$$

where I and II refer to the two types of Mn sites. In each of these planes, the lons are ferromagnetically coupled. The coupling between $I I_{a}$ and $I I_{b}$ is ferromagnetic, while the I- $I I_{a}$ and $I-I I_{b}$ coupling is antiferromagnetic giving the ferrimagnetic behavior since the magnetizations of the $I$ and II sublattices are not equivalent. Doping with Or substitutes a smaller ion in the Mn site, reduces the value of $\underline{c}$ slightly, and affects the exchange interaction between the sublattice planes. In Fig. 2, we present saturation magnetization data showing a sharp drop to a lower value below a well defined temperature which is a function of the concentration of or. At this point, the lattice parameter has reached a critical size $\underline{c} \approx 6.53$. 8 , the II $_{\mathrm{a}}-\mathrm{II}_{\mathrm{b}}$ coupling changes to antiferromagnetic. and the material becomes a pure antiferromagnet. The magnitude 


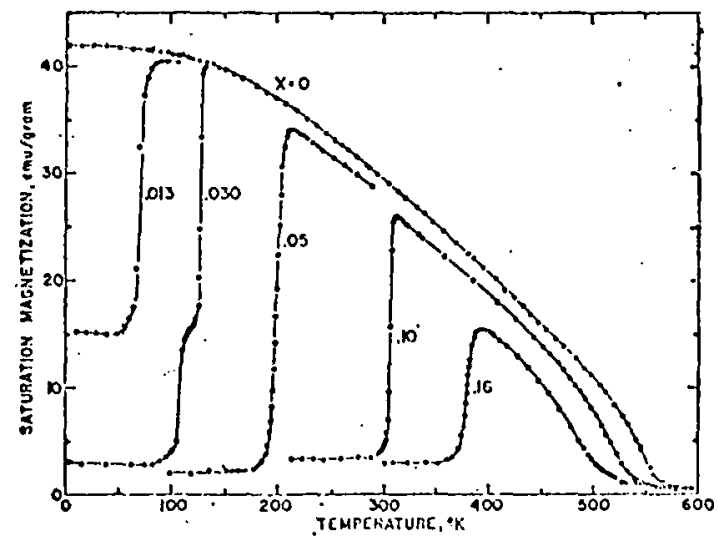

FIG. 2. Magnetization vs temperalurc; for $\mathrm{Mn}_{2-2} \mathrm{Cr}_{2} \mathrm{Sb}$; data on cooling; $I I \perp c$.

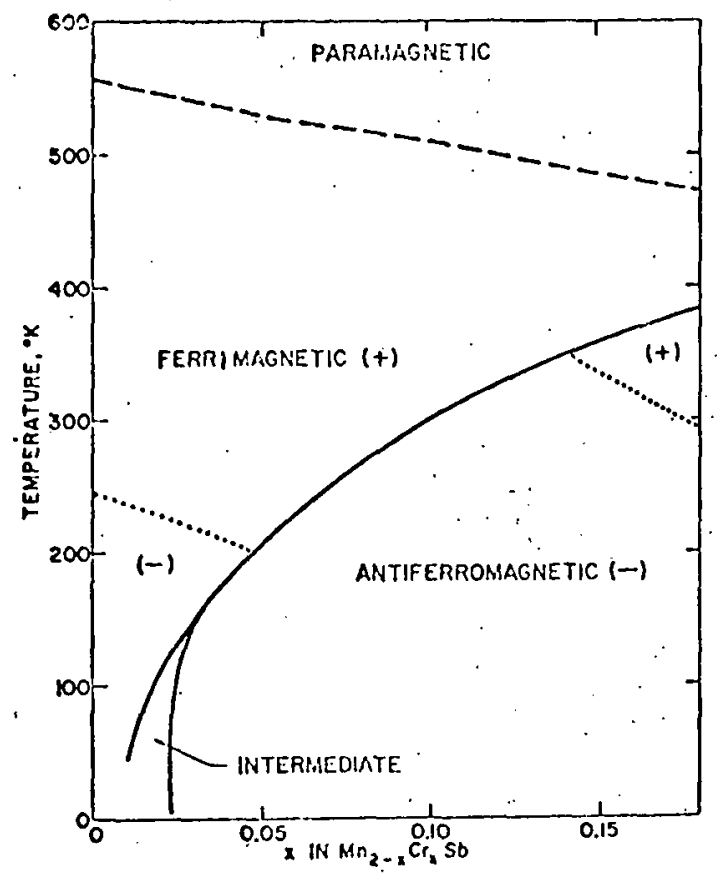

FIc. 3. Phase diagram for the Cr-modificd Mn ${ }_{2}$ Sl) system. 
of c and a change discontinuously, but no change in crystal symmetry is detectable, establishing this as a firstworder phase transition. A specific heat anomaly ${ }^{14}$ and a change in resistivity ${ }^{15}$ have also been measured. To account for this transition, Kittel ${ }^{16}$ has developed a thermodynamic theory in which the exchange interaction is coupled with the elastic strain of the lattice and can change sign at a critical value of the lattice parameter.

Flg. 3. shows a complete phase diagram of $\mathrm{Mn}_{2-\mathrm{x}} \mathrm{Cr}_{\mathrm{x}} \mathrm{Sb}$ 。 There is an additional intermediate state in which the moments are ordered in a spiral configuration. This. accounts for the intermediate value of the magnetization seen in Fig。2. An extention of Kittel's calculation 17 shows that transitions to this intermediate state are also firstorder。 Fig。 2 shows a small residual mag netization in the antiferromagnetic phase. This arises from MnSb which unavoidably precipitates out when the material is prepared. The signs $(\infty)$ and (+) in the phase diagram (Fig. 3) refer to the sign of the anisow tropy energy. The dotted line dividing these regions is the locus of the rotation of the easy axis of magnetization. 


\section{Sublattice Magnetization}

To account for the temperature dependence of the saturation magnetization, Guillaud proposed a model in which the efectronic states of the two mn sites are temperature dependent. However, in $1948 \mathrm{Né}^{18}{ }^{18}$ developed a molecular field theory of ferrimagnetism which successfully accounted for the temperature variation of the magnetization without requiring a change in the electronic states. In this theory each magnetic site is coupled to an effective field from each of the two sublattices... The magnetization of each sublattice .M. (I) and M.(II) is obtained from simultaneous solutions of

$$
\begin{aligned}
M(I)= & N g_{I}{ }^{S} \mu_{\beta} \\
& \times B_{S_{I}}\left[\left(g_{I}{ }^{s} I \mu_{\beta} / k T\right)(\eta / 2)(\alpha \cdot M(I)+M(I I))\right]
\end{aligned}
$$

and

$$
\begin{aligned}
M_{I}(I I)= & N g_{I I} S_{I I} \mu_{\beta} \\
& \times B\left[\left(g_{I I} S_{I I} \mu_{\beta} / \mathrm{kT}\right)(\eta / 2)(M:(I)+\beta M(I I))\right]
\end{aligned}
$$

where. $N$ is Avogadro.'s number, $k$ is the Boltzman constant, $g_{I}$ and $g_{I I}$ are the effective g-values, $s_{I}$ and $s_{I I}$ are the spin magnitudes of the $M n(I)$ and $M n(I I)$ sites respectively, and $B_{S}$ is the Brillouin function. Solutions to these equations have been made by Darnell et al: 13 and Tonegawa ${ }^{19}$. where they evaluated the parameters $\eta, \alpha$, and $\beta$ by fitting the solutions to neutron diffraction and saturation magnetization data. It was found that 
the total magnetiantion M(II). $\approx$ M(I) is very insensitive to the exact values of these parameters. We shall. be interested in comparing our sublattice frequency measurements with these calculations.

\section{Magnetic Domain. Structure}

We will briefly consider the domain structure since it plays an important role in the NMR. The domain structure in the a-c plane of a single crystal of $\mathrm{Mn}_{2} \mathrm{Sb}$ has been studied from photographs of the Bitter pattern. 20 The domains are roughly rectangular: $100-200 \mu$ long parallel to the coaxis and $20-40 \mu$ wide. However, the size of domains is very sensitive to the size and shape of the crystal. 21 : For a metal particle to be a single domaln, it must be smallèr than several hundred ang stroms. Since our sample of $\mathrm{Mn}_{2} \mathrm{Sb}$ is: powdered to $44 \mu$ particles, it is probably multidomain.

The magnetization curve of powdered $\mathrm{Mn}_{2} \mathrm{Sb}$ is shown below. The steep slope at low fields is assom ciated with displacement of $180^{\circ}$ domain walls as domains magnetized parallel to the field grow in size. At higher fields the dominant magnetization process is the rotation of domains. At fields above $5 \mathrm{kOe}_{2} \mathrm{Mn}_{2} \mathrm{Sb}$ is essentially saturated and the, Bloch walls are eliminated。 


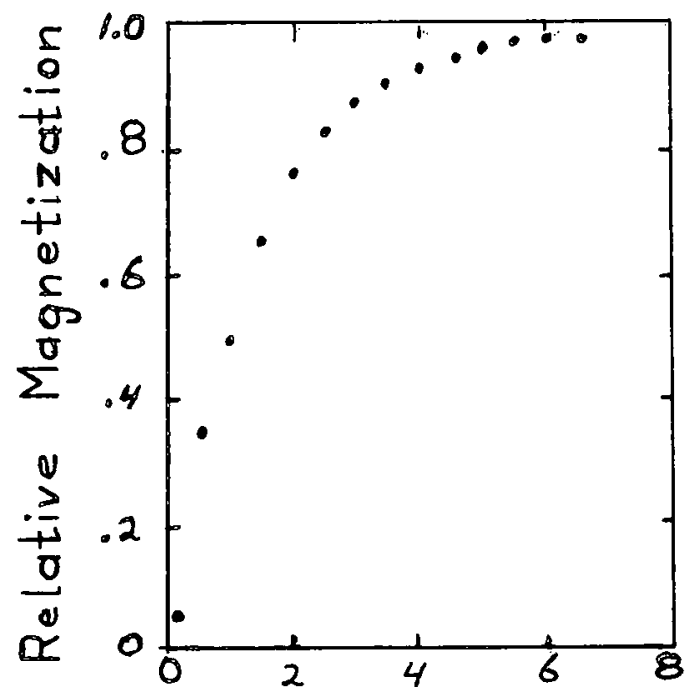

Applied Magnetic Field, kOe

\section{Hyperfine Interactions}

We now consider the magnetic hyperfine interaction of the nucleus with the electron magnetization. Since most of our NMR measurements are made on the Mn sites, we shall concentrate our attention on them and mention $\mathrm{Sb}$ only briefly.

Manganese lies in the middle of the iron series where the $3 d$ band is being filled. The $3 d$ band is very. narrow and has a high density of states at the Fermi. level. The much broader $4 \$$ band overlaps the $3 d$ band. Since the $3 d$ band is near the surface of the atom it is sensitive to its environment, and the orbital moment is usually completely quenched by cxystaline fields。 In $\mathrm{Mn}_{2} \mathrm{Sb}$ we expect the electronic' configuration of the two Mn sites to be a mixture of $3 d^{5} 4 s^{2}$ and $3 d^{7}$ configuram tions. This introduces a nonmzero orbital momentum and 
a mixing of the dand s electron bands. our inability to describe the exact electronic configuration makes accurate and quantitative interpretation of experimental results difficult.

The one electron theory of magnetic hyperfine interactions was first developed by Fermi 22 from the Dirac relativistic theory。 The well mnown Hamiltonian for this interaction is

$$
\begin{aligned}
H_{h f}=-g_{I} \mu_{g} \mu_{N}\{8 \pi / 3 \delta(r) \vec{S} \cdot \vec{I} & +\frac{(\vec{I}-\vec{S}) \cdot \vec{I}}{r^{3}} \\
& \left.+\frac{3(\vec{S} \cdot \vec{r})(\vec{I} \cdot \vec{r})}{r^{5}}\right\},
\end{aligned}
$$

where $I_{\theta} S_{\theta}$ and $I$ are angular momentum operators of the electron orbital, electron spin, and nuclear spin respecm tively, $\mu_{\beta}$ and $\mu_{\mathrm{N}}$ are the Bohr and nuclear magneton, and $g$ and $g_{I}$ are the electronic and nuclear spectroscopic splitting factors. The last two terms are called the orbital and dipolar terms. They are analogous to the classical expressions for the interaction between two point dipoles $g_{I} \mu_{\mathrm{N}} I$ and $g \mu_{\beta} \mathrm{s}$ and between the point dipole $g_{I} \mu_{N} I$ and a circulating charge with angular momentum.I。 For s electrons, these terms are zero; and. we are left with the first term, the Fermi contact interaction, which depends on the existence of a nonvanishing wave function of the s electron at the nucleus. 
Although this theory has been considered successful in accounting for the hyperfine splitting in atomic spectra and the Knight shift in metals, it fails dramatically in. the case of $\mathrm{Mn}^{++}$in the $3 d^{5}, 6 \mathrm{~s}$ configuration. By Hund's rule $I=0$ and only the contact term contributes to the hyperfine field. There are no unpaired electrons, the $5 / 2$ spin comes from the $3 d^{5}$ electrons, so the contact term is also zero. However, fields up to $700 \mathrm{kOe}$ have been measured for $\mathrm{Mn}^{++}$in insulators.

The inadequecy of the onemelectron theory was recognized as early as 1933 by Fermi and Segre。 ${ }^{23}$ The task of developing a satisfactory many-electron theory is still..far from complete. The subject is so vastly complex that we shall briefly mention only results pertinent to our studyo. Most of this material will be taken from two excellent review articles by Watson and Freeman。24, 25

Iet us first consider the charge distribution of closed electron shells in the core of an atom. Sternheimer and cowworkers ${ }^{26}$ suggested that spin polarization of these electron shells would contribute to the magnetic hyperfine interaction. The exchange interaction between the polarized unpaired 3d electrons and a core s electron will depend on their relative: orientation. Thus, the spin up and spin down s electron wave functions will be distorted differently, splitting the 
pairs of core electrons and producing a net spin density at the nucleus, The Fermi contact interaction from. these shelis is now non-vanishing. The field resulting from this core electron contact term is given by

$$
H_{c}=(8 \pi / 3) g \mu_{\beta} \mathrm{S} \sum_{\substack{\text { electron } \\ \text { shelis }}}\left\{\rho_{\uparrow}(0)-\rho_{\downarrow}(0)\right\}_{0}
$$

where $\rho(0)=|\psi(0)|^{2}$ the spin density at the nucleus and $S$ is the total. spin of the ion in question. This core palarization interaction is the dominant contribution to the hyperfine field in both metals and insulators. Detailed calculations of $\mathrm{H}_{\mathrm{c}}$ for the $\mathrm{Mn}^{++}$free ion have been made by Watson and Freeman, and their results compare favorably with measurements for ions in salts. The net value of $\mathrm{H}_{c}$ is the sum of contributions of opposing sign from different electron shells o. For reference we will consider the direction of the electron magnetization. to be positive: The is and $2 \mathrm{~s}$ shells contribute a negative field. The $3 \mathrm{~s}$ shell produces a positive field, and because of its proximity to the $3 d$ electrons we might expect this term to dominate. However, the 3 s and $3 d$ bands overlap introducing competing tendencies that lead to a small positive contribution. Because of the large size of the individual contributions, small errors in calculation can result in large errors in the value of $\mathrm{H}_{C}$, and even change its sign. We expect calculations of $\mathrm{H}_{C}$ to be very sensitive to 
environmental influences on the electron wave functions especially in. the $3 \mathrm{~s}$ and $3 \mathrm{~d}$ overlap contribution.

Calculation of the $\mathrm{Mn}^{++}$free fon yields $\mathrm{H}_{\mathrm{C}} \approx-700 \mathrm{kOe}_{8} 27$ while measurements of $\mathrm{Mn}^{++}$in hydrated salts by Abragam $^{28}$ give $\mathrm{H}_{\mathrm{C}} \approx-650 \mathrm{kOe}$, where $\mathrm{H}_{\mathrm{C}}$ is the total effective field seen by the nucleus. Experimental measurements show that $H_{C}$ is very sensitive to the ion ${ }^{\prime} s$ environment. Calculations involving all four s electron shells for the neutral Mn atom (in the $3 d^{5} 4 s^{2}$ configuration) yield $\mathrm{H}_{\mathrm{c}} \approx-168 \mathrm{kOe}$, while the most recent data by Winkler give $H_{c} \approx-126 \mathrm{kOe}^{29}$ The data also show a monotonic increase in $\mathrm{H}_{c}$ in the iron series as predicted by. theory. These successes are remarkable considering the possibility of large errors.

It is clear that $H_{C}$ is the dominant contribution to the effective field seen at the nucleus. However, there are many other hyperfine field contributions in a. ferromagnetic metal. Most of these are positive and compete with the core polarization; moreover these are difficult to estimate accurately. Early calculations actually predicted'a positive value for $\mathrm{H}_{c}$, but these were contradicted by later measurements in most transition metals.

There are two other sources of hyperfine field from the core electron's in $\mathrm{Mn}_{2} \mathrm{Sb}$. Since the symmetry of the crystal is less that cubic, there may be a dipolar 
field from the 3d electrons. There may also be an orbital field which is proportional to the unquenched angular momentum of the $3 d$ electrons, since the covalent bonding and mixing of $3 \mathrm{~d}$ and $4 \mathrm{~s}$ electrons may result in a net angular momentum.

Polarization of the $4 \mathrm{~s}$ conduction electrons may also contribute a hyperfine field via the Fermi contact interaction. They can be polarized either by exchange interaction with the core d electrons, by covalent mixing of $4 \mathrm{~s}$ and $3 \mathrm{~d}$ electrons, or by some admixture of s electron wave function into the d band. Finally, the conduction electrons can be polarized by coupling to the 3d electrons via the Zener-Ruderman-Kittel-Kosuya-Yosida $(Z-R-K-K-Y)^{30}$ mechanism. This mechanism produces an oscillation of the spin density in the conduction bond due to the mixing of $4 \mathrm{~s}$ electrons in different $k$ states polarized about a $3 d$ electron site.

A large hyperfine field is observed at the $\mathrm{Sb}$ nucleus even though it is a non-magnetic atom. The important mechanisms responsible for this are:

(1) the admixture of 3d-spin density from the Mn site distorting and polarizing the $\mathrm{Sb}$ closed shell wave functions.

(2) polarization of the $s$ electron in $\mathrm{Sb}$ by the exchange field of the $\mathrm{Mn} 3 \mathrm{~d}$ electrons, and 
(3) polarization of the conduction electrons by the $(Z-R-K-K-Y)$ exchange interaction. From our NMR measurements on...Mn $\mathrm{Mn}_{2} \mathrm{Sb}$ at $77^{\circ} \mathrm{K}$ with no external field, we find the effective field at the nucleus to be approximately 137, 120, and $240 \mathrm{kOe}$ for the $M n(I), M n(I I)$, and $S b$ sites respectively. We note that $H_{c}$ for the Mn sites is considerably less than the value for a free $\mathrm{Mn}^{++}$ion, indicating the extent to which covalent bonding and other factors decrease $\mathrm{H}_{\mathrm{c}}{ }$. The fact that fields at the two Mn sites differ only by $10 \%$ suggests that the electron configuration is quite similar.

Nuclear Magnetic Resonance in Ferromagnetic Metals

A system of nuclear moments with gyromagnetic ratio $\gamma$ in a magnetic field $H_{0}$ will have transitions induced between its magnetic energy levels by an electromagnetic field $\mathrm{H}_{q}$, applied perpendicular to $\mathrm{H}_{0}$ at the Larmor frequency $\omega_{0}$. This resonance condition results. in power absorption from the oscillating field and change in the population of different energy levels. The longitudinal and transverse relaxation times of the nuclear magnetization are designated by $T_{1}$ and $T_{2}$ respectively。 The power absorbed by a nuclear spin system in a homogeneous magnetic field is given by 


$$
\rho\left(\omega_{0}\right)=\frac{\chi_{0} \omega_{0}^{2} \mathrm{~T}_{2} \mathrm{H}_{1}^{2}}{1+\gamma^{2} \mathrm{H}_{1}^{2} \mathrm{~T}_{1} \mathrm{~T}_{2}} .
$$

This produces a Lorentzian line shape with a width at half power $(\Delta \omega)_{\frac{1}{2}}=1 / T_{2}$, which is the natural line width of the spin system. For weak rf fields such that $\gamma^{2} \mathrm{H}_{1}^{2} \mathrm{~T}_{1} \mathrm{~T}_{2} \ll 1_{\text {, }}$

$$
\rho\left(\omega_{0}\right)=X_{0} \omega_{0}^{2} \mathrm{~T}_{2} \mathrm{H}_{1}^{2}
$$

while for strong rf fields

$$
\rho\left(\omega_{0}\right)=X_{0} H_{0}^{2} / T_{1}
$$

This latter case is the saturation condition in which the shape of the resonance signal becomes distorted and independent of $\mathrm{H}_{1}$ 。

I In many systems the natural line shape is not observed. An inhomogeneous distribution of local magnetic fields will broaden the NMR line to a width

$$
(\Delta \omega)_{\frac{1}{2}}=\gamma_{\Delta H_{r m s}}
$$

where $\Delta H_{r m s}$ is the root mean square of the local field fiuctuation. An important source of this inhomogenelty is the magnetic dipole interaction which will produce a Gaussian field distribution.

In ferromagnetic materials the total magnetic field, $\mathrm{H}_{e}$, seen by the nuclear moment in an applied 
external field $\mathrm{H}_{0}$ can be written in the form

$$
\overrightarrow{\mathrm{H}}_{\theta}=\overrightarrow{\mathrm{H}}_{0}+\overrightarrow{\mathrm{H}}_{\mathrm{D}}+\overrightarrow{\mathrm{H}}_{\mathrm{L}}+\overrightarrow{\mathrm{H}}_{\mathrm{hf}}
$$

We have already discussed the sources of the hyperfine field $H_{h f}$ from the local electron magnetization.

Within a magnetic specimen, the uniform orientation of magnetic dipoles will produce a Lorentz field given by

$$
\overrightarrow{\mathrm{H}}_{\mathrm{L}}=(4 \pi / 3) \overrightarrow{\mathrm{M}}_{\mathrm{S}}
$$

The demagnetizing field. $\mathrm{H}_{D}$ arises from the free magnetic poles induced on the surface of the magnetized specimen and has the form:

$$
\begin{array}{ll}
\overrightarrow{\mathrm{H}}_{\mathrm{D}}=-\overrightarrow{\mathrm{H}}_{0} & \text { for } \mathrm{H}_{0} \leqslant \mathrm{NM}_{\mathrm{S}}, \\
\overrightarrow{\mathrm{H}}_{\mathrm{D}}=-\mathrm{N} \overrightarrow{\mathrm{M}}_{\mathrm{S}} & \text { for } \mathrm{H}_{0} \geqslant \mathrm{NM}_{\mathrm{S}} .
\end{array}
$$

The demagnetizing factor $N$ is a function of the shape of the material. In cgs units $N=4 \pi$ for a thin disk perpendicular to $M_{S}, 4 \pi / 3$ for a sphere, and 0 for $a$ long rod parallel to $\mathrm{M}_{\mathrm{S}^{\circ}}$.

As we apply an external static field $\mathrm{H}_{\mathrm{o}}$ to magnetize our sample, we expect the resonance frequency ito change by $\Delta \omega=\gamma\left(\mathrm{H}_{0^{-}}-, \mathrm{NM}_{\mathrm{S}}\right)$ : In the case of co studied by Gossard and Portis $31 \Delta \omega / 2 \pi$ was calculated to be $200 \mathrm{kHz}$ while a shift of less than $20 \mathrm{kHz}$ was measured. In a multidomain particle the domain walls will be shifted to a new equilibrium position by the applied field. 
Thus, the nucled in the domain wall, which are the dominant source of the NMR signal are shielded from the external field. Once $H_{0}$ is large enough to eliminate the $180^{\circ}$ walls, normal shifts in the resonance frequency are observed.

The skin depth $\delta$ limits the penetration of rf field into a conducting material. From Maxwell's equations we get

$$
\delta \approx \frac{1}{\sqrt{2 \pi \mu \omega \sigma}}
$$

where $\sigma$ is the conductivity and $\mu$ is the permeability。 For Fe at $100 \mathrm{MHz}$, assuming that $\mu(\mathrm{rf})=\mu($ low freq.), $\delta=1$ micron. We have not found any satisfactory calculation of $\delta$ in. magnetically ordered material appropriate to the frequency region of interest. In any case, we find that powdering our sample to $44 \mu$ particles is sufficient to produce a large NMR signal. Contrary to expectation, the NMR signal in Co is very large, making it a trivial resonance to detect. By observing the onset of saturation and calculating the saturation parameter, $\gamma_{1}^{2} \mathrm{H}_{1}^{2} \mathrm{~T}_{1} \mathrm{~T}_{2}$, Portis and Gossard 31 concluded that $\mathrm{H}_{q}$ must be larger than the applied $\mathrm{rf}$ field by a factor of $10^{3}$. This large enhancement is due to the fact that the resonant rf field at the nucleus is driven indirectly by the external field via 
the strong hyperfine coupling with the electron magnetization. We now discuss the nature of this enhancement.

Iet us first consider the effect of coherent rotation of the domain magnetization by $\mathrm{H}_{q}$ in the Iimit that the electron and nuclear spins are decoupled。 32 The electron magnetization $\overrightarrow{\mathrm{M}}_{\mathrm{S}}$ will rotate until the torque exerted by $\mathrm{H}_{\mathrm{q}}$ equals the torque exerted by $\mathrm{H}_{0}+\mathrm{H}_{A^{2}}$ where $\mathrm{H}_{A}$ is the anisotropy field. Then we have

$$
\overrightarrow{\mathrm{H}}_{q} \times \overrightarrow{\mathrm{M}}_{\mathrm{S}}=\left(\overrightarrow{\mathrm{H}}_{\mathrm{A}}+\overrightarrow{\mathrm{H}}_{\mathrm{O}}\right) \times \overrightarrow{\mathrm{M}}_{\mathrm{S}} \text {. }
$$

If $\mathrm{H}_{\hat{q}}$ is perpendicular to $\mathrm{M}_{\mathrm{S}}$ and the angle of rotation $\theta$ is small, then

$$
H_{1} M_{S}=\left(H_{A}+H_{O}\right) M_{S} \sin \theta \approx\left(H_{A}+H_{O}\right) M_{S} \theta
$$

and

$$
\theta=\mathrm{H}_{q} /\left(\mathrm{H}_{\mathrm{A}}+\mathrm{H}_{\mathrm{O}}\right) \text { 。 }
$$

The transverse rf field $\mathrm{H}_{\text {in }}$ seen by the nucleus with hyperfine field $\mathrm{H}_{\mathrm{hf}}$ will be

$$
\begin{aligned}
\mathrm{H}_{1 \mathrm{n}} & =\mathrm{H}_{1}+\theta \mathrm{H}_{\mathrm{hf}} \\
& =\mathrm{H}_{1}+\left[\mathrm{H}_{1} /\left(\mathrm{H}_{\mathrm{A}}+\mathrm{H}_{0}\right)\right] \mathrm{H}_{\mathrm{hf}} \\
& =\mathrm{H}_{1}\left[1+\mathrm{H}_{\mathrm{hf}} /\left(\mathrm{H}_{\mathrm{A}}+\mathrm{H}_{0}\right)\right] . \\
& =\eta_{\mathrm{D}} \mathrm{H}_{1}
\end{aligned}
$$

where $\eta_{D}$ is the domain enhancement factor. For Co we have $H_{h f} \approx 217 \mathrm{kOe}$ and $\mathrm{H} \approx 1$ kOe, giving $\eta_{\mathrm{D}} \approx 200$. 
This is too small to account for the observed enhancement. The effect of domain wall motion can be considered by using the simple model of a spherical particle with a single domain wall. 31 The wall displacement $x$ due to $\mathrm{H}_{1}$ will be limited by the demagnetization energy。

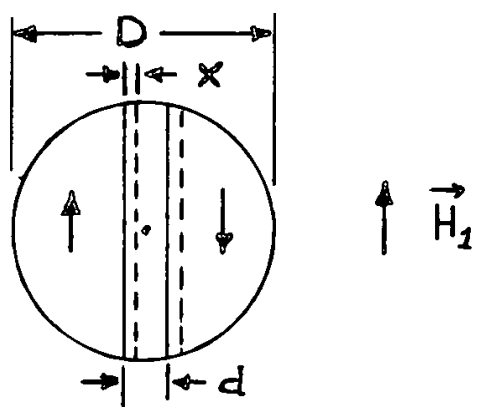

The energy gained by motion of the domain wall is

$$
2 \mathrm{M}_{S} \mathrm{H}_{1} \Delta V \approx \frac{1}{2} \mathrm{M}_{S} \mathrm{H}_{\uparrow} \mathrm{D}^{2} \mathrm{x}
$$

The net change in the magnetization of the particle is

$$
\Delta M=M_{S} \therefore \Delta V / V=\frac{M_{S} \pi x D^{2} / 2}{\pi D^{3} / 6}=3 M_{S} x / D \cdots
$$

If as an approximation, we consider this distributed over the whole particle with demagnetizing factor $N_{\text {, }}$ then the demagnetizing energy is

$$
E_{D} \approx \int_{0}^{M} H d(M V)=V \int_{0}^{M}(N M) d M=\frac{1}{2} V_{N M^{2}} .
$$

Substituting in $M=\Delta M$ we have

$$
\begin{aligned}
E_{D} & =\frac{1}{2} N\left(\pi D^{3} / 6\right)\left(3 M_{S} x / D\right)^{2} \\
& =(3 \pi / 4) D x^{2} M_{S}^{2} .
\end{aligned}
$$


The maximum displacement $x_{m}$ will be given by the equilibrium condition

$$
E_{D}=\frac{1}{2} M_{S} \cdot H_{q} D^{2} X_{m}
$$

which yields

$$
\begin{aligned}
& (3 \pi / 4) D x_{m}^{2} N_{s}^{2} \approx \frac{1}{2} M_{s} H_{q} D^{2} x_{m} \\
& x_{m} \approx 2 D H_{q} / 3 N M_{s} .
\end{aligned}
$$

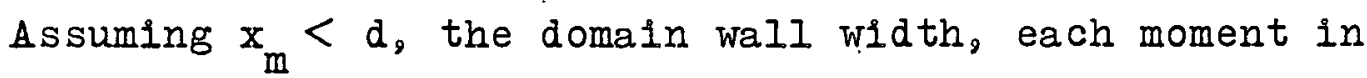
the wall will rotate through the angle

$$
\theta=\pi x_{m} / d
$$

The transverse rf field will be

$$
H_{1 n}=\theta H_{h f} \approx\left[\frac{2 \pi D H_{h f}}{3 d N M_{S}}\right] H_{1} \text {, }
$$

giving an enhancement factor

$$
\eta_{\omega} \approx(D / d) \mathrm{H}_{\mathrm{hf}} / \mathrm{NM}_{\mathrm{S}}
$$

As before $\mathrm{H}_{\mathrm{hf}} \approx 10^{5}$ oe and $\mathrm{NM}_{\mathrm{S}} \approx 10^{3}$ oe, but now we have the additional: factor $D / d \approx 10$. Thus, $\eta_{\omega} \approx 10 \eta_{D} \approx 10^{3}$ and this accounts for the strength of the NMR signal observed by Porti.s and Gossard. 
Spin Echo Formation

Our NMR measurements are made primarily by detecting spin echoes. When short pulses of intense rf magnetic field at the resonance frequency are applied, spontaneous nuclear spin induction signals or "echoes" will appear due to constructive interference of precessing moment vectors. This technique has been widely developed since first reported by $E$. I. Hahn in 1950.33 Hahn's paper provides a detailed account of the fundamentals of the subject.

The effect of an oscillating field with amplitude $2 \mathrm{H}_{q}$ and frequency $\omega$ can be most readily analysed by breaking it into two rotating field components with frequency $\omega$ and $-\omega$ and amplitude $\mathrm{H}_{q}$. It can be shown that the field component rotating in the opposite sense to the precessing moment can be neglected in the NMR analysis.

To understand the motion of a nuclear moment in a static field $H_{0}$ and $r f$ field $H_{q}$ with frequency $\omega_{8}$ it is simplest to transform to a coordinate system rotating at $-\omega .{ }^{34}$ Below we show the fields in such a reference frame with $H_{1}$ directed along the $\mathrm{y}$-axis and $\mathrm{H}_{0}$ parallel to the z-axis. Notice that all fields are now static and the effective magnetic field is

$$
\overrightarrow{\mathrm{H}}_{\mathrm{eff}}=\left(\mathrm{H}_{0}-\omega / \gamma\right) \hat{z}+\mathrm{H}_{q} \hat{y}
$$




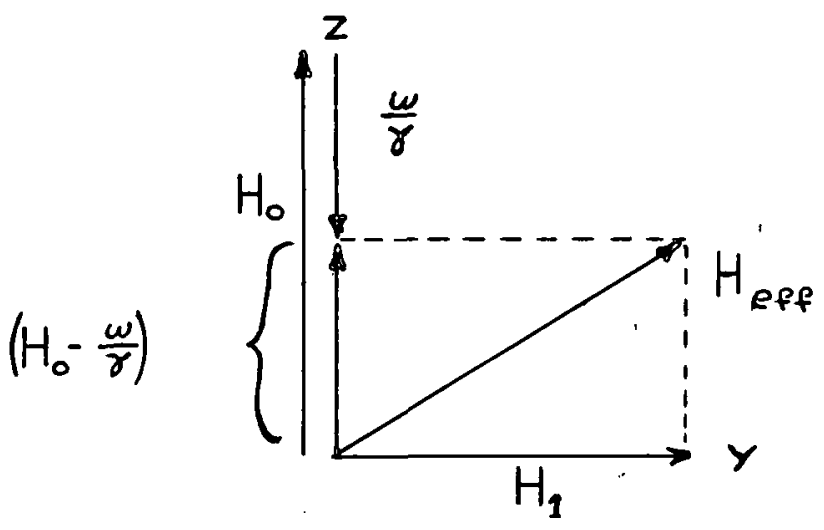

The magnetic moments will precess in a cone about $\mathrm{H}_{\mathrm{e} f \mathrm{f}}$ tangent to the $z$-axis. At the resonance condition with $\omega=\omega_{0}=\gamma_{H_{0}}$, we will have $\mathrm{H}_{\text {eff }}=\mathrm{H}_{1}$ and the moments will precess in the $x z-p l a n e$. When $\mathrm{H}_{1}$ is turned off, $\mathrm{H}_{\text {eff }}=0$ and the moments will be stationary in the rota ting frame but precess about the $z$-axis in the laboratory frame at the Larmor frequency $\omega_{0}$.

If $\mathrm{H}_{1}$ is applied as a short pulse of duration $t_{\omega} \ll T_{2}, T_{1}$, an ensembie of spins with macroscopic moment $\vec{M}$ will precess an angle

$$
\theta=\omega_{1} t_{\omega}=\gamma_{H_{q}} t_{\omega}
$$

from its equilibrium position parallel to the z-axis。 After a $90^{\circ}$ pulse, i。e, $\theta=\pi / 2, \vec{M}$ is in the $x y-p l a n e$ and the free precession of the moment will. induce an rf signal. During this time, relaxation and possibly diffusion processes will diminish the transverse component of $\vec{M}$ as individual nuclear spins get out of phase with one another or return to thermal equilibrium. Neglecting diffusion, the total transverse relaxation. 
time will be given by

$$
\frac{1}{\mathrm{~T}_{2}}=\frac{1}{\mathrm{~T}_{2}^{\prime}}+\frac{1}{\mathrm{~T}_{1}}
$$

where $T_{2}^{\prime}$ is a spin-spin relaxation time.

A more general situation is an ensemble of spins with a distribution $g(\Delta \omega)$ of Larmor frequencies centered about $\omega_{0}$ due to static field inhomogeneities. After a $90^{\circ}$ pulse, the spin system in a frame rotating at $\omega_{0}$ will fan out, reducing the magnitude of $\vec{M}_{\text {. After }}$ a time $t$, where $2 \pi /(\Delta \omega)_{\frac{1}{2}}<t \ll \mathbb{T}_{1}$, the spins will be isotropically distributed in the $x y$-plane and $\vec{M}=0$. This produces a free precession signal with a characteristic decay time $T_{2}^{*} \approx 1 /(\Delta \omega)_{\frac{1}{2}}$. If a second ( $r e$ focusing) $r f$ pulse is applied, the spin system may be brought back into phase. The simplest case to describe is $180^{\circ}$ refocusing pulse. The moment $\overrightarrow{\mathbb{M}}$ starts in thermal equilibrium parallel to the z-axis and is rotated $90^{\circ}$ to the $x$ axis. The individual moments fan out in the $x y-p l a n e$ and $\vec{M}$ becomes zero as they become isotropically distributed. After a time $\tau_{\text {, where }} t_{\omega}<\tau<\mathbb{T}_{1}, \mathbb{T}_{2}$, a $180^{\circ}$ pulse rotates the moments about the y-axis。 The nuclear spins that have not undergone transverse relaxation will fan back into phase at time $2 \tau$ along the $-x$ axis, inducing a rf signal (echo) with frequency $\omega_{0}$ and magnitude proportional to $\vec{M}(2 T)$. The spin echo shape 
is a function of $g(\Delta \omega)$ and has a width $T_{2}^{*}$. As long as $\mathrm{T}_{2}^{*} \ll \mathrm{T}_{2}, \mathrm{~T}_{1}$, and $\omega_{1} \gg 1 / \mathrm{T}_{2}^{*}$, the echo amplitude is independent of $\mathrm{T}_{2}^{*}$. When the transverse relaxation is exponential, $T_{2}$ is measured directly by plotting the logarithm of the echo amplitude as a function of $2 \tau$.

These measurements are much simpler and more direct than those using the shape of the steady-state resonance line. In that case, the natural line width, which is inversely proportional to $\mathrm{T}_{2}$, is often distorted by saturation or broadened by magnetic field inhomogeneity. With spin echo measurements, the driving field $\mathrm{H}_{q}$ is absent, and it.is the envelope of the echo amplitude and not the echo shape that is measured.

Spin echo formation can be generalized to the condition of two equal exciting pulses. The formation of the echo becomes more complicated since all the spins do not converge on the $-x$ axis but produce a polarization of spin density along the $-x$ axis. From Hahn's analysis 33 the voltage of the echo signal, neglecting terms associated with diffusion, is given by

$$
\begin{aligned}
& V(t) \approx M_{0} \sin \left(\omega_{1} t_{\omega}\right) \sin ^{2}\left(\omega_{1} t_{\omega} / 2\right) \\
& \quad X \exp \left\{\frac{-(t-2 T)^{2}}{2\left(T_{2}^{*}\right)^{2}}-\frac{t}{T_{2}}\right\},
\end{aligned}
$$

where $M_{0}$ is the equilibrium macroscopic momentum vector and $t=0$ at the beginning of the first rf pulse. 
The maximum echo amplitude will be obtalned when $\omega_{1} t_{\omega}=120^{\circ}$. If the two rf pulses are coherent, there will be interference terms between the free precession signal immediately following the $r f$ pulse and the echo signal unless $\tau \gg T_{2}^{*}$, preventing overlap of these signals.

If a third identical pulse is applied at time $\mathbb{T}$, we will get more echoes at times $T+\tau, 2 T-2 T, 2 T-\tau$; and 2T.We will describe the formation of the echo at $T+\tau$, called the stimulated echo. After the first $90^{\circ}$ pulse, the spins are in the $x y-$ plane. The second $90^{\circ}$ pulse rotates the spins to the $x z-p l a n e$. During the primary echo at $2 \gamma$, the spins focus into a figure 8 pattern projected on a sphere.tangential to the poles of the z-axis and centered on the $x$ axis. During the interval between $2 \tau$ and $T$ these spins precess in cones about the z-axis. To understand the echo at $T+\tau$, let us follow two particular spins. A spin with Larmor frequency $\omega=\omega_{0}$ will not fan out but be fixed along the $+x,-z$, and $-x$ axis at the end of each of the three $90^{\circ}$ pulses respectively. The spins, such that $\left(\omega-\omega_{0}\right) r=90^{\circ}$, will be along the $y$ axis at the end of the second pulse and fan out in the $x y$-plane in the following interval. The third $90^{\circ}$ pulse rotates them into the $y z-p l a n e$, and they focus to a figure 8 pattern at $T+\Psi$. Other spins will follow a more complicated pattern but contribute to a net polarization of spins 
along the $x$ axis to produce the echo at $T+\tau$. The amplitude of this echo for $T \gg \gamma$ is

$$
V(T+\tau) \approx \frac{1}{2} M^{0} \sin ^{3}\left(\omega_{1} t_{\omega}\right) \exp \left(-T / T_{1}\right)
$$

We now have a direct and convenient way to measure $\mathbb{T}_{1}$;

In certain situations, the spin echo signal is modulated 35 Ihis is associated with the existence of closely spaced energy levels in the spin system. There are two distinctiy different modulation patterns which we will now describe.

Consider a system of two non-interacting spin ensembles with similar frequency distribution but slightly different Larmor frequencies $\omega^{\prime}$ and $\omega^{\prime \prime}$. Each forms an echo configuration in two frames of reference rotating at frequencies $\omega^{0}$ and $\omega^{\prime \prime}$. The induction signals from $M^{\prime}$ and $M^{\prime \prime}$ produce a beat signal of frequency $\left|\omega^{\prime}-\omega^{\prime \prime}\right|$. At $t=2 \tau$ the two frames are in phase, and the total induction is the sum of the two:

$$
\mathrm{V}_{\text {Tot }}(2 \tau)=\left[\mathrm{V}^{\mathrm{P}}(2 T)^{2}+\mathrm{V}^{n}(2 T)^{2}\right]^{\frac{1}{2}} \quad \vdots
$$

The beat pattern is symmetric about the echo maximum. The other pattern is a slower modulation of the maximum spin echo amplitude as a function of $2 T$. This modulation cannot be a simple interference of different Larmor frequencies, since, as we have shown, the echo maxima at $2 \gamma$ is due to the sum of the contributing spin 
groups and independent of their Larmor frequencies. The slow modulation is the result of a splitting and mixing of degenerate levels by quadrupole or Zeeman perturbation. If there are two closely spaced energy levels which are not coupled by offodiagonal matrix elements, there will not be any modulation of the spin echo maximum amplitude. In the next section we will consider the quadrupole splitting of zeeman levels, which is the situation we find in $\mathrm{Mn}_{2} \mathrm{Sb}$. Measurements of the period of modulation can give us information on the splitting of degenerate levels even when the individual lines are so broadened as to be unresolvable by steady state resonance detection.

\section{Quadrupole Modulation of Spin Echoes}

Neglecting non scalar components, the quadrupole interaction along a principal axis of the crystalline potential $V(r)$ for nucled with spin I and quadrupole moment $Q$ is

$$
H_{Q}=\frac{e Q}{4 I(2 I-1)}\left(3 I_{z}^{3}-I^{2}\right) V_{z Z} \text {. }
$$

Applying Laplace's equation $\nabla^{2} V=0$, we have

$$
\mathrm{v}_{\mathrm{xx}}+\mathrm{v}_{\mathrm{y} \mathrm{y}}+\mathrm{v}_{z z}=0
$$

For uniaxial symmetry where $\mathrm{V}_{\mathrm{xx}}=\mathrm{V}_{\mathrm{yy}}$ s we must have $\mathrm{v}_{\mathrm{zz}}=-2 \mathrm{v}_{\mathrm{xx}}{ }^{\circ}$ 
In the limit where the Zeeman field is strong, we can treat the quadrupole interaction by perturbation theory. If we set $\mathrm{V}_{z z}=\mathrm{eq}$, then the energy levels of the system are given by

$$
\begin{aligned}
E_{m} & =-\hbar \omega_{0} m+\frac{e^{2} q Q}{4 I(2 I-1)}\left[3 m^{2}-I(I+1)\right] . \\
& =-\hbar \omega_{0} m+\hbar m^{2} a+\text { const. },
\end{aligned}
$$

where

$$
a=\frac{3 e^{2} q Q}{4 I(2 I-1)}
$$

The quadrupole interaction will produce $2 I$ equally spaced lines centered about the Larmor frequency $\omega_{0}=\gamma_{0} H_{0}$ The separation between adjacent lines is

$$
\Delta \nu_{Q}=a / \pi
$$

The density matrix formalism is the simpiest and most direct way to calculate the spin echo amplitude. We describe the spin system in the rotating frame by the density matrix $\rho(t)$ whose motion satisfies the equation

$$
\frac{\dot{d} \rho(t)}{d t}=-i[\mathcal{H}, \rho(t)],
$$

where $\mathscr{H}$ is the Hamiltonian in the rotating frame. The If signal induced in the laboratory frame by the precessing magnetization is proportional to $s(t)$ given by 


$$
s(t)=\operatorname{Tr}\left[\rho(t) I_{+}\right]
$$

with

$$
I_{+}=I_{x}+i I_{y}
$$

The most detalled calculations of spin echo modulation have been recently made by Hisashi Abe et al.36 They considered a system of spin I nuclei which have a large Zeeman energy $h \omega_{0} I_{z}$ with inhomogenous broadening about a center frequency $\omega_{00}$ given by the function $f\left(\omega_{0}\right)$ with width $\delta \omega_{0}$ and a quadrupole interaction haI $I_{z}^{2}$ such that $\omega_{0} \gg$ a. They averaged over the ensemble of spins to get the total inducedirf echo signal

$$
S(t)=\int f\left(\omega_{0}\right) s(t) d \omega_{0}
$$

If the nuclear spin system is in thermal equilibrium with the lattice, then

$$
\begin{aligned}
\rho(0) & \propto \exp \left\{\hbar\left(\omega_{0} I_{z}+a I_{z}^{2}\right) / k T\right\} \\
& \approx 1+\left(\hbar \omega_{0} / k T\right) I_{z}
\end{aligned}
$$

assuming that $\mathrm{kT} \gg \omega_{0} I_{z} \gg \mathrm{aI}_{z}^{2}$. The constant term does not interest us since

$$
\operatorname{Tr}\left(I_{+}\right)=0 \text {, }
$$

so we can write

$$
p(0)=I_{z}
$$


If we apply the rf field. With a frequency $\omega$.and strength $H_{1}=\omega_{1} / Y$ along the $y$-axis, then we have

$$
H=\Delta \omega I_{z}+a I_{z}^{2}+\omega_{1} I_{y}
$$

and

$$
\rho(t)=U \rho(0) U^{-1}
$$

where

$$
U=\exp (-i \mathscr{H} t) \text { and } \Delta \omega=\left|\omega_{00}-\omega\right| \ll a .
$$

In its most general form this is written as

$$
U=\left(\begin{array}{rrrr}
A & O & E & F \\
-C & B & D & E \\
E & -D & B & C \\
-F & E & -C & A
\end{array}\right)
$$

In the limit that $a / w_{1} \rightarrow 0$, $U$ becomes a simple rotation operator about the $y$ axis. For the special cases of $90^{\circ}$ and $180^{\circ}$ pulses, we have 37

$$
U_{90}=1 / 2 \sqrt{2}\left(\begin{array}{rrrr}
-1 & -\sqrt{3} & -\sqrt{3} & -1 \\
\sqrt{3} & 1 & -1 & -\sqrt{3} \\
-\sqrt{3} & 1 & 1 & -\sqrt{3} \\
1 & -\sqrt{3} & \sqrt{3} & -1
\end{array}\right)
$$

and 


$$
U_{180}=\left(\begin{array}{rrrr}
0 & 0 & 0 & -1 \\
0 & 0 & 1 & 0 \\
0 & -1 & 0 & 0 \\
1 & 0 & 0 & 0
\end{array}\right)
$$

After the first rf pulse with a duration $t_{\omega}$,

$$
\sigma\left(t_{w}\right)=U_{I} I_{z} U^{+} \text {. }
$$

Since, $\sigma\left(t_{\omega}\right)$ is a Hermitian matrix, it can be written as a linear combination of angular momentum operators 38 as follows

$$
\begin{array}{rlr}
\sigma\left(t_{\omega}\right) & =a_{1} I_{z}+a_{2} I_{z}^{3} & \text { (diagonal elements) } \\
& \left.+a_{3} I_{+}+a_{4} \frac{1}{2}\left(I_{z} I_{+}+I_{+} I_{z}\right)\right\} \begin{array}{c}
\text { (elements one } \\
\text { above diagonal) }
\end{array} \\
& +a_{5} I_{z} I_{+} I_{z} \\
& +\ldots
\end{array}
$$

plus higher order terms for further off-diagonal elements. Abe et al. show how these elements contribute to the multiple spin echoes that are observed after a single rf pulse pair. The diagonal elements contribute to the free precession signal after the second rf pulse, elements one off-diagonal the first echo, and so on. When $I=5 / 2$, as it is in Mn, $U$ is a $6 \times 6$ matrix and we can expect at most five echoes. If $U$ is a perfect rotation operator for an angle $\theta=\omega_{1} t_{\omega}$ then

$$
\sigma\left(t_{\omega}\right)=I_{z} \cos \theta+I_{x} \sin \theta
$$

and only the free precession and first echo signal 
will be produced. In our work we observed the first two echoes at times $2 \tau$ and $3 \tau$.

The induced rf signal after the second pulse is obtained by calculating

$$
S(t)=\operatorname{Tr} \quad U_{I V} U_{I I I} U_{I I} \sigma\left(t_{\omega}\right) U_{I I}^{-1} U_{I I I}^{-1} U_{I V^{I}+}^{-1},
$$

where U UI, UIII, UIV are the appropriate operators for the intervals before, during, and after the second pulse respectively. For the first echo Abe et al. obtain

$$
\begin{aligned}
\mathrm{S}^{(1):(2 \tau)=} & -4|\mathrm{D}|^{2}-6|\mathrm{C}|^{2} \\
& +8 \sqrt{3}\left|\mathrm{BE} \mathrm{E}^{*}\right| \cos \left(2 \mathrm{a} \tau+\delta \mathrm{BE}^{*}\right) \\
& +6\left|\mathrm{DF^{* }}\right| \cos \left(4 \mathrm{a} \tau+\delta \mathrm{DF^{* }}\right) .
\end{aligned}
$$

The echo is modulated with a fundamental frequency $a / \pi$. If the second $r f$ pulse is a $180^{\circ}$ pulse, then $B$ and $E^{*}$ are zero and the fundamental modulation is $2 \pi / a$.

The results for $I=5 / 2$ show the existence of five multiple echoes each modulated by various harmonics of the fundamental frequency $a / \pi$. Neglecting relaxation mechanisms, the amplitudes for the two echoes at $t=2 \tau$ and $3 \uparrow$ are given by

$$
\begin{aligned}
E^{(1)}(2 \tau)= & c_{0}^{(1)}+c_{1}^{(1)} \cos \left(2 a \tau+\delta_{1}^{(1)}\right) \\
& +c_{2}^{(1)} \cos \left(4 a \tau+\delta_{2}^{(1)}\right)+\underset{\text { high harmonic }}{\text { terms }}
\end{aligned}
$$

and 


$$
\begin{aligned}
\mathrm{E}^{(2)}(3 \gamma)= & c_{1}^{(2)} \cos \left(2 a \tau+\delta_{1}^{(2)}\right) \\
& +c_{3}^{(2)} \cos \left(6 a \tau+\delta_{3}^{(2)}\right)+\underset{\text { terms }}{\text { igh harmonic }}
\end{aligned}
$$

respectively. The $C^{\prime} s$ are functions of the matrix

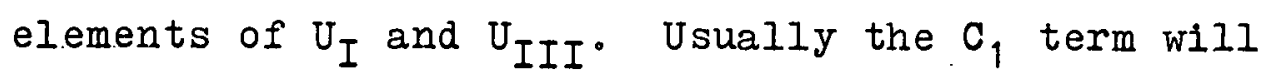
dominate, and the modulation period will be $\mathrm{T}_{\mathrm{m}}=\pi / \mathrm{a}$. Combining this with the quadrupole splitting $\Delta \nu_{Q}$, we have

$$
T_{m} \Delta \nu_{Q}=(\pi / a)(a / \pi)=1
$$

This gives us a way to measure the quadrupole interaction directly from the spin echo envelope modulation.

The higher harmonic terms contain the far offdiagonal matrix elements and represent mixing of widely spaced multiplet lines. Their amplitude will depend on the exciting condition, i. e., the shape and width of the $r f$ pulses. If the frequency spectrum is narrow, then only adjacent multiplets will be excited, and the fundamental modulation frequency will dominate. 
NMR Frequency and Local Magnetization

The general form for the hyperfine Hamiltonian between a nuclear spin $I$ and electron $\operatorname{spin} S$ is

$$
\mathcal{H}_{\mathrm{hf}}=\vec{I} \cdot[\mathrm{A}] \cdot \overrightarrow{\mathrm{S}}
$$

where [A] is a second order tensor hyperfine coupling constant. For an isotropic [A], as is the case for an s state Ion, we have

$$
H_{h f}=A \vec{I} \cdot \vec{S}
$$

Due to the strong exchange interaction in a magnetically ordered system, the fluctuations in $s_{z}$ about 1 ts expectation value $\left\langle S_{Z}\right\rangle$ will be predominantly near the exchange frequency $\left(\sim 10^{12} \mathrm{~Hz}\right)$ and will average to zero during the nuclear precession period $\left(\sim 10^{-8}\right.$ sec). Therefore, to first order the resonance frequency will be

$$
\omega_{0}=\frac{1}{\hbar}|A\langle S\rangle|
$$

A second order calculation by deGennes et al. 39 on a ferromagnetic system yields

$$
\omega=\omega_{0}\left[1-\eta\left(m_{0} / M_{0}\right)\right],
$$

where $\eta$ is the enhancement factor, $m_{0}$ the nuclear magnetization and $M_{O}$ the electronic magnetization. If, because of a low anisotropy field, the electronic magnetization is able to follow the nuclear magnetization via the 
I.S.interaction, then the effective field seen by the nuclei is reduced, pulling the NMR frequency down. Since $\eta_{\text {wall }} \gg \eta_{\text {domain }}$ the resonance lines may be split.into two lines $\omega_{\text {domain }}>\omega_{\text {wall }}$. A careful study of this effect has been made by Heeger and Houston. 40 Saturation by the rf field reduces $m_{0}$ to zero, and the NMR lines shift back to $\omega_{0}$ As long as the condition $\eta\left(m_{0} / \mathbb{M}_{0}\right)<<1$ is maintained, the NNR frequency will be proportional to $\left|A\left\langle S_{Z}\right\rangle\right|$ 。

If the behavior of $A(T)$ is known, NMR frequencies corrected for changes in volume can be used to study the temperature dependence of the sublattice magnetization of the nuclear site. The hyperfine coupling of the $\mathrm{Mn}^{++}$ion has been studied extensively by Walsh et al. ${ }^{41}$ They find that the value of A for $\mathrm{Mn}^{++}$bound in a variety of crystals can be fitted up to $500^{\circ} \mathrm{K}$ by

$$
A(T)=A(0)\left(1-C T^{3 / 2}\right),
$$

with $0 \approx 10^{-6}-10^{-8}$. Usually $A(T)$ is dominated by thermal expansion effects. However, for these $\mathrm{Mn}^{++}$ions $\left[\frac{\partial \ln A}{\partial \ln r}\right]_{T}>0$ and $A(T)$ should increase slightly with temperature. Therefore, the temperature dependence of $A(T)$ must be dominated by thermal fluctuations. Theoretical calculations by Símánek, Orback, Vai I1 Huang, and Ohho-Yuan Huang $42-44$ successfully explain this behavior. They account for the reduction of the hyperfine 
interaction by considering the orbit-lattice coupling and covalent mixing of excited configurations of the form $3 \mathrm{~d}^{4}$.ns. into the $3 \mathrm{~d}^{5}$ ground state by acoustic and optical phonons.

Even at $200^{\circ} \mathrm{K}$ the drop in $\mathrm{A}(\mathrm{T})$ from $\mathrm{A}(0)$ is less than $1 \%$, so the temperature dependence of the NMR frequency will closely follow the local magnetization $\mathbb{M}_{S}(T) \propto\left\langle S_{Z}\right\rangle$. At low temperatures we expect the deviation of the magnetization to be given by spin wave theory which predicts that

$$
\left\langle S_{z}(T)\right\rangle=\left\langle S_{z}(0)\right\rangle\left[1-a T^{3 / 2}-b T^{5 / 2}-\ldots\right] .
$$

Combining this with the behavior of $A(T)$ we have

$$
\omega(T)=\omega_{0}\left[1-(0+a) T^{3 / 2}-b T^{5 / 2}-\ldots\right] .
$$

Measurements of $\mathrm{Mn}^{++}$in $\mathrm{MnFe}_{2} \mathrm{O}_{4}$ by Heeger and Houston 40 show that $a \approx 10^{-5}>0$, so that $\omega(T)$ can be used to verify spin wave theory directly。

The domain structure introduces more complications. A study of thermal excitation of the Bloch wall by Winter 45 shows that the magnetization is not uniform throughout the wall and that it is less than the domain magnetization. Measurements by Narath ${ }^{46}$ in $\mathrm{CrI}_{3}$ confirm this and show that as the temperature increases $\omega(T)$ wall decreases faster than $\omega(T)$ domain $^{\circ}$ However, recent measurements by Yasuoka et al. ${ }^{47}$ on single domain Co particles find that for temperatures up to $600^{\circ} \mathrm{K}$ 


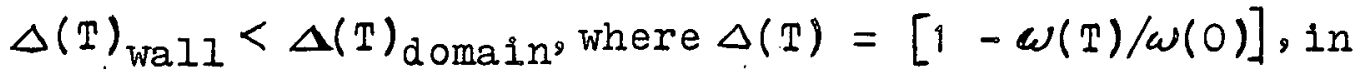
contradiction to Winter ${ }^{\mathrm{s}}$ theory. There is no clear understanding of this result or of its implications for current theories of Bloch walls.

\section{Nuclear Relaxation}

The strong ordering interactions in a ferromagnetic metal make the nuclear relaxation times very short. In the domain walls there are additional mechanisms for even faster relaxation. Present theory is unable adequately to account for measured values of $T_{1}$ inside the domains. We shall give here a review of the mechanisms involved in the various relaxation processes.

The strong nuclear spin-spin coupling is due to the Suhl-Nakamura interaction. 48 Here the spins are coupled indirectly via the hyperfine coupling to electronic spin waves. This is similar to the RudermanKittel interaction which couples magnetic moments via the conduction electrons. Because this interaction is an indirect coupling, we would not expect it to produce a pure exponential decay, which is observed in most cases. Weger et al. 49 suggest that static broadening from field inhomogeneity, especially in or near a domain wall, will partially decouple the nuclei and produce an exponential relaxation. The interaction in the domain wall is enhanced by the wall magnons due to thermal 
fluctuation. According to Winter's calculation ${ }^{45}$ the relaxation is enhanced by the ratio of the domain anisotropy to the apparent wall anisotropy, and this gives results in agreement with the experiment.

Measurements of $T_{\mathfrak{q}}$ show a faster non-exponential decay followed by a longer exponential decay. The rapid decay is associated with the nuclei In the domain walls. Winter ${ }^{45}$ has studied the thermal fluctuations of domain walls dampened by eddy currents. Weger 50 has shown that the fluctuations of the transverse field due to this motion account for the shortest relaxation observed in $\mathrm{Ni}, \mathrm{Co}_{8}$ and Fe. These relaxation times are nearly temperature independent. Since the fluctuation is a function of position in the wall, there will be a continuous range of relaxation times giving rise to the non-exponential decay.

The longer pure exponential decay from the nuclei in the domains is not even qualitatively understood yet. $T_{1}$ has the temperature dependence $T_{q} T=$ constant which suggests the Korringa conduction electron mechanism. Here the nuclei relax directly to the s state conduction electrons via the Fermi contact interaction. Integration over the distribution of the electrons at the Fermi surface produces the characteristic temperature dependence 


$$
\frac{1}{T_{1}} \propto T\left\langle\left|u_{K}(0)\right|^{2}\right\rangle_{T} \rho^{2}\left(E_{F}\right),
$$

where $\left|u_{K}(0)\right|^{2}$ is the spin density at the nucleus and $\rho\left(E_{F}\right)$ the density of states at the Fermi surface。

Although this is a resonable description for non-magnetic metals, $T$ is is too large for ferromagnetic systems such as $\mathrm{Ni}, \mathrm{Co}$, and $\mathrm{Fe}$ where $\mathrm{T}_{1} \mathrm{~T}$ is $0.115,0.080$, and $2.5 \sec ^{\circ} \mathrm{K}$ respectively。

Toru Moriya ${ }^{51}$ has made a general study of the various possible relaxation mechanisms to account for these small values of $T$, Although the Fermi contact interaction is significant, it is not of primary importance. Magnetic dipole, electric quadrupole, and Fermi contact interaction via core polarization are all negligible: Although the spin wave system provides a thermal reservoir for energy relaxation, the only process proportional to temperature requires a direct absorption or emission of a spin wave as a nuclear spin flips. Unfortunately, spin wave frequencies are much larger than the Zeeman frequency making this process negligible. other spin wave processes such as mixing with phonons have not been studied theoretically but are not expected to be important。

Moriya finds that orbital relaxation is the dominant process. Here electrons are scattered by nuclear spins without changing their own spins. The coupling to the 
fluctuating orbital current of the d electrons is calculated using a tight binding approximation and is found to be large due to the high density of states at the Fermi surface. However, Walstedt et al. ${ }^{52}$ point out that the rates are over-estimated and are not in agreement with experiment. This is in contrast with orbital calculätions on nonferromagnetic metals where the relaxation rates are in excess of those observed. Also, $T_{1}$ does not increase as one goes through the transition metals from Mn to Ni as an orbital mechanism would predict, nor is $T_{1} \approx T_{2}$, which one expects assuming the absence of appreciable spin-orbit coupling. At present there is no other mechanism adequate to explain this confusing situation. 


\section{Chapter III}

Experimental Design and Procedure

\section{Materials}

Pure and homogenous samples of $\mathrm{Mn}_{2-\mathrm{x}} \mathrm{Cr} \mathrm{Sb}$ are very difficult to prepare. The Mn-Sb phase diagram 53 shows that close to the Mn 2 S.b phase $(5.1 .05-52.57$ $\left.w t \% ~ S b, m p=913^{\circ} \mathrm{C}\right)^{54}$ there is a eutectic point $\left(45 \mathrm{wt} \% \mathrm{Sb}, \mathrm{mp}=880^{\circ} \mathrm{C}\right)^{54}$ and an $\mathrm{MnSb}$ phase $(61.61$ $\left.68.91 \mathrm{wt} \% \mathrm{Sb}, \mathrm{mp}=872^{\circ} \mathrm{C}\right)$. Both the $\mathrm{MnSb}$ and eutectic will be present when $\mathrm{Mn}_{2} \mathrm{Sb}$ is prepared under nonequilibrium conditions. By adding an excess of Mn or small amounts of In, the MnSb precipitate can be reduced. However, it usually amounts to $0.5-3.0 \%$ by volume and produces the residual magnetization measured when $\mathrm{Mn}_{2-\mathrm{x}^{\mathrm{Cr}} \mathrm{x}} \mathrm{Sb}$ is antiferromagnetic.

A study of the microstructure of crystals of $\mathrm{Mn}_{2} \mathrm{Sb}$ by Wolf and Hanlon ${ }^{20}$ using a Bitter pattern technique confirmed the presence of these phases. In the eutectic, pure $\mathbb{M n}$ is dispersed in a $\mathrm{Mn}_{2} \mathrm{Sb}$ matrix. The MnSb exists as a separate precipitated phase. In Cr-doped $\mathrm{Mn}_{2} \mathrm{Sb}$ the $\mathrm{Or}$ is not homogeneously distributed. I.t concentrates around the Mn particles leaving the rest of the eutectic matrix deficient in Cr. This has the effect of broadening the magnetic transitions 
by several degrees since the transition temperature is a function of $\mathrm{Cr}$ concentration. Careful annealing improves the situation but does not achieve a completely homogeneous sample.

We prepared samples of $\mathrm{Mn}_{2-\mathrm{x}} \mathrm{Cr}_{\mathrm{x}} \mathrm{Sb}$ by the following procedure.o. The proper proportions of $99.999 \% \mathrm{Sb}$, 99.9\% Cr, and 99.999\% Mn powder were mixed and sealed in a quartz tube. The mixture was heated to $100,0^{\circ} \mathrm{C}$ for one hour then quenched, producing a clean, spongy metal structure. This material was put in an alumina crucible, sealed in a glass tube, and annealed for three days a.t $400-700^{\circ} \mathrm{C}$. These samples showed a sharp F-AF transition $\left(5-10^{\circ} \mathrm{C}\right.$ wide). We found that $\mathrm{Mn}_{2} \mathrm{Sb}$ is easily ignited, so we took the precaution of grinding the material under a liquid to keep it cool. When the powder was annealed, the transition was often washed out completely.

Much effort was expended in improving this procedure with little success. in sharpening the F-AF transition or obtaining the intermediate spiral phase (I)。 Discussions with workers at du Pont confirmed that impurities are an especially serious problem when handling fine powders. We obtained from du Pont a single crystal sample with very sharp transitions. 55 Grinding the material to successively finer particles produced a successively broader transition. Although 
annealing helped to narrow it the transition width never returned to its original sharpness. We suspected that the effect of the grinding was to introduce strains into the sample and to create a range of local transition temperatures. Although we expected the annealing to remove these strains, impurities introduced while treating the powder may also have broadened the transition.

The NMR work reported here is the result of studies on two samples. One is pure $\mathrm{Mn}_{2} \mathrm{Sb}$ prepared in the manner we have described. After being annealed at $500^{\circ} \mathrm{C}$ for 3 days, the bulk material was ground to 325 mesh size under acetone. About $8 \mathrm{~g}$ of the powder was cast in paraffin to form an annular cylinder $2.4 \mathrm{~cm}$ o.d.g $1.5 \mathrm{~cm} \mathrm{1.d.2,} \mathrm{and} 1.7 \mathrm{~cm} \mathrm{10ng.}$

The second sample is $\mathrm{Mn}{ }_{1.97^{\mathrm{Cr}}} \mathrm{C}_{0.03} \mathrm{Sb}$, obtained from du Pont. 55 single crystals of the material showed two sharp magnetic transitions: $F-I$ at $128^{\circ} \mathrm{K}$ and I-AF at $110^{\circ} \mathrm{K}$, each $2-3^{\circ}$ wide. We were given pieces of the melt from which these crystals were grown. We put this material in an alumina crucible inside a pyrex tube, outgassed at $100^{\circ} \mathrm{C}$ for one day, and pumped to below $10^{-5}$ Torr. It was annealed at $600^{\circ} \mathrm{C}$ for $2 \frac{1}{2}$ days and slowly cooled to room temperature in 10 hours. The sample then showed a sharp F-AF transition, $4-5^{\circ}$ wide, at $120^{\circ} \mathrm{K}$ with only a slight trace of an intermediate phase. The material was carefully broken into single 
crystal pieces. These were cleaved perpendicular to the c-axis with a razor blade. The sample was split into flakes as thin as could be handled with fingers, about $0.25 \mathrm{~mm}$ thick. The flakes were stacked into piles maintaining constant c-axis orientation. Approximately 6 .g were cast in paraffin to form an annular cylinder $2.6 \mathrm{~cm}$ long with the c-axis parallel to the axis of the cylinder. We call this the bulk sample.

\section{Magne.tometer}

our macroscopic magnetization measurements were made with a Foner vibrating sample magnetometer. 56 In this instrument the material to be studied is placed in. a homogeneous magnetic field. The sample and a reference magnetic dipole are vibrated at some suitable audio frequency. The audio frequency signals induced by these vibrating dipoles are picked up by two pairs of stationary colls. These two af signals are mixed $180^{\circ}$ out of phase, amplified, and then detected. The magnetization of the sample is proportional to the amplitude of the reference signal required to produce a null in the detected signal.

In our apparatus we used a $40 \mathrm{~W}$ driver vibrating a rod at $150 \mathrm{~Hz}$. The sample, approximately $0.09 \mathrm{~cm}^{3}$, was mounted on the end of the rod and placed in the $2^{\prime \prime}$ gap between the 8 " diam pole faces of a magnet with 
a maximum field of $16 \mathrm{kOe}$. A heater and thermocouple mounted in a copper block around the sample placed inside a vacuum can immersed in a liquid nitrogen bath provided continuous temperature control down to $63^{\circ} \mathrm{K}$, the triple point of nitrogen. The reference dipole was a small coil mounted on the top of the rod. It was powered by a very stable constant current supply whose current level is controlled linearly by a precision 10 turn potentiometer. The magnitude of the reference dipole was proportional to the setting on the potentiometer dial. The af signals from two identical pickup coils were mixed in a transformer, amplified, and detected by a phase sensitive bridge, driven at 150Hz. The output of the bridge was filtered and measured on a microammeter. The magnetization measurements were made simply by adjusting the potentiometer to produce a null reading on the ammeter. Although the reference dipole could easily be calibrated, we were only interested in relative measurements.

The magnetometer was sensitive touchanges about $1 / 3 \%$ of full scale or approximately 0.002 emu. Although this instrument design is capable of greater sensitivity, our sensitivity was more than sufficient to measure the changes in magnetization in $\mathrm{Mn}_{2-x} \mathrm{Cr}_{\mathrm{x}} \mathrm{Sb}$ 。 Circuit diagrams and more details on the construction of this apparatus are given in appendix $\mathbf{A}$. 
Spin Echo Spectrometer Design

A block diagram of the spectrometer used for the spin echo measurements is shown in Fig. 4. The spectrometer is a balanced bridge design similar to those used in microwave spectroscopy. The function of the hybrid junction is to route rf power to the sample and to isolate the detection circuit from the rf oscillator. The $r f$ echo signal from the sample is sufficiently strong that it can be amplified and displayed directly on an oscilloscope. The hybrid junction is a broadband device which has been avallable only in the last few years. It allows one to employ microwave circuit techniques in the radio frequency region.

The pulse generator (GR 1395-A) has five delaypulse units capable of producing a trainof three pulses. Both pulse duration and spacing can be varied. These range from $0.1 \mu \mathrm{sec}$ to $100 \mathrm{sec}$, and they are continuously variable over any decade. The pulse train can be triggered for a single sweep or be given a variable repetition rate from $3 \mathrm{~Hz}$ to $1.2 \mathrm{MHz}$.

The pulsed rf oscillator uses a GE6442 planar triode. This tube is speciflcally designed for operation as a plate pulsed, grounded-grid oscillator at frequencies up to $5000 \mathrm{MHz}$. It features a compact ceramic construction with cylindrical symmetry。 Electrical contact to the tube is made via spring finger 


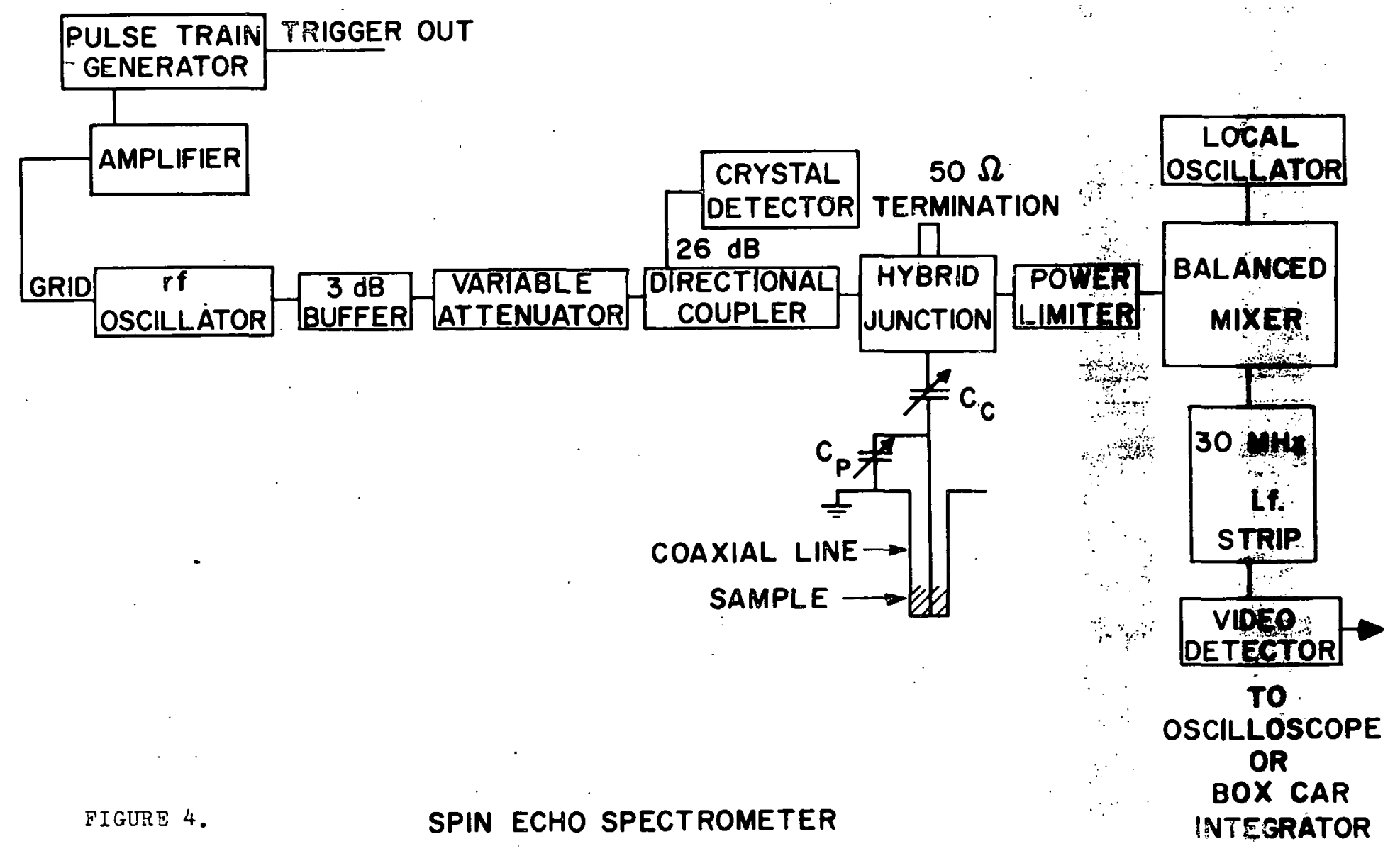


contact rings which minimize concentrations of $r f$ current to assure high power, high frequency operation. When the plate is pulsed with $3 \mathrm{kV}$, it can produce $4 \mathrm{~kW}$ pulses at $200 \mathrm{MHz}$ with a duty factor of 0.001 . The circuit diagram of our rf oscillator is presented in Fig. 5. To avoid the difficulties of switching high voltages, we used a pulsed grid design. In this mode of operation, the oscillator produced incoherent pulses of $\mathrm{rf}$ power. The two important design problems were to prevent continuous oscillations, which would damage the tube, and to form sharp stable pulses with maximum power. Some of our solutions to these problems seemed to depend on the particular physical arrangement of our circuit. The oscillator was

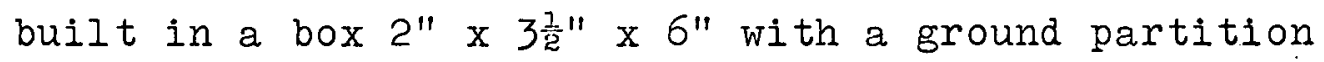
separating the plate and cathode circuits. The grid is ac grounded via a $600 \mu F$ bypass capacitor built directly into the partition.

The cathode was biased to $+12 \mathrm{~V}$ with respect to ground to prevent oscillation when the grid is at ground potential. However, as higher voltage was applied to the plate, continuous oscillation persisted after the positive potential on the grid was removed. During the application of the positive pulse, the grid first goes positive to initiate oscillation then drops back to ground during the rest of the oscillation period. 

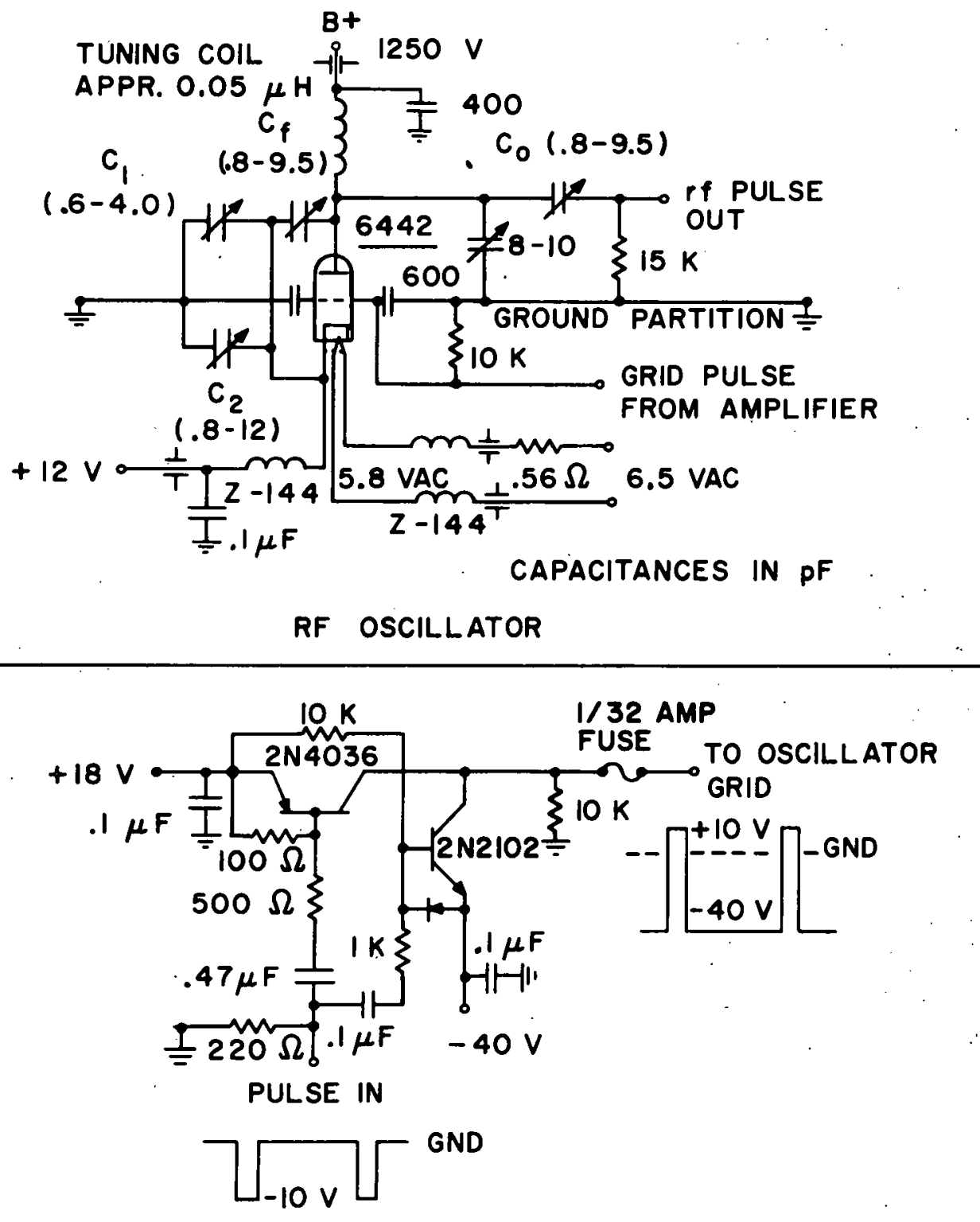

FIGURE 5. GRID PULSE AMPLIFIER 
Therefore, to assure a rapid turn off we applied a large negative bias to the grid. .The amplifier shown in Fig. 5 was built to perform this. The $-10 \mathrm{~V}$ pulse from the pulse generator turns on the $2 \mathrm{~N} 4036$ producing a bias with respect to ground of approximately $+12 \mathrm{~V}$ on the grid. At the end of the pulse, the $2 \mathrm{~N} 2102$ is turned back on to apply a negative bias to the grid. A bias from $-20 \mathrm{~V}$ to $-40 \mathrm{~V}$ worked best.

The final shape and stability of the rf pulses depends on the adjustment of several capacitors. The need for capacitors $\mathrm{C}_{1}$ and $\mathrm{C}_{2}$ was discovered in an ad hoc fashion. They not only sharpen the cut off of the rf pulse but they also increase the power. Our only explanation for this is that by rapidly dissipating rf energy they reduce the tendency for the circult to sustain cw oscillation after the pulse period. It now can be: operated at a hlgher power level and still produced sharp pulses. The position of both $C_{j}$ and $C_{2}$ is critical; $c_{1}$ should be as close to $c_{f}$ as possible.

Even. with these precautions care had to be taken when turning on the oscillator. The minimum voltage, $500 \mathrm{~V}$, from the power supply (Fluke 404M) could be safely applied to the plate. The capacitors $\mathrm{C}_{1}, \mathrm{C}_{2}, \mathrm{C}_{f}$, and $C_{0}$ were then adjusted to achieve a stable pulse. As the plate potential was increased in steps of $100 \mathrm{~V}$, $c_{f}$ was reduced and $c_{0}$ increased to maintain a sharp 
- pulse. We operated at a maximum plate potential of $1250 \mathrm{~V}$ and obtained pulses with $0.2 \mu$ sec rise time and $350 \mathrm{~W}$ maximum power. Going to higher voltages made. stable pulses difficult to achleve and did not cause an appreciable increase in the power.

The power level dropped off rapidly as the circuit was tuned to lower frequencies. We were able to maintain $350 \mathrm{~W}$ by using a series of inductors of increasing number of turns starting with a $0.06 \mu \mathrm{H} 3$ turn coil. Each one was used for a 10-15 MHz region. For a given frequency we used the largest inductor that could be tuned to that frequency. It seems that the oscillator requires a high plate impedance to maintain a high power level.

The hybrid junction (Anzac H1) is a low frequency device equivalent to a microwave hybrid tee with a bandwidth of 5-1000 $\mathrm{MHz}$. Input power at one end port will be equally divided into the two side ports. If the impedance at the two side ports are equal, i.e., the bridge is balanced, then any power reflected back into the junction will return to the input port. If this balanced condition is not satisfied, half of the unbalanced portion of the reflected power will appear at the fourth port. When the bridge is balanced, the isolation between the two end ports is $50 \mathrm{~dB}$. The hybrid junction is used in a balanced bridge 
circuit. One arm is a standard $50 \Omega$ termination while the other is a tuned coaxlal Iine connected to the hybrid junction by a $50 \Omega$ coaxial cablemalince the impedance of the side ports of the junction is $100 \Omega$, there will be some power reflected due to the impedance mismatch. The impedance of the tuned coaxial line is matched to 50 at the frequency of the rf oscillator. This matches the coaxial line to the $50 \Omega$ cable and allows maximum power to be transmitted to the sample. It also balances the bridge protecting the balanced mixer from the pulses of high rf power. The optimum design would be to make both arms of the bridge $100 \Omega$ and eliminate the power reflection due to impedance mismatch at the hybrid junction. The power limiter (MA 8445-T11) is added for extra protection in case of a gross unbalance. The $3 d B$ buffer provides some isolation to the rf oscillator from the power reflected back from the hybrid junction. Unfortunately the spin echo signal generated in the coaxial. Ine is also divided so that only half of the power is detected. The balanced mixer, an Anzac HFK-2ORW with 10-1000 MHz bandwidth, mixes the signals from the hybrid junction and the local oscillator. The output of the mixer is a signal with frequency equal to the difference of the two input signals. By setting the frequency of the local oscillator to $\pm 30 \mathrm{MHz}$ of the NMR frequency, the spin echo signal is amplified by the $30 \mathrm{MHz}$ 1.f. amplifier. The i.f. amplifier 
(IEL Model IMM-2-30-02-50) has a noise factor of $1.4 \mathrm{~dB}$, a. gain of $136 \mathrm{~dB}$, and a bandwidth of $2.2 \mathrm{MHz}$. It features a very fast recovery time, less than $1 \mu \mathrm{sec}$, making it suitable for detecting. fast pulses, although some low level ringing is observed for a few microseconds longer. Built into the i.fostrip is a video detector which employs a diode to measure the amplitude of the 1.f. signal. The maximum output of the detector is a $40 \mathrm{~V}$ pulse. Between 2 and $30 \mathrm{~V}$ the output is proportional to the voltage of the 1.f. input signal. Below $2 \mathrm{~V}$ the detection is square law, i.e., the output voltage is proportional to the power of the input signal. We operated the detector in the linear region so that the output is proportional to the $r f$ voltage from the hybrid junction. From the output of the video detector, the shape of the spin echo can be displayed directly on an oscilloscope.

The coaxial line functions as an inductance $I$ in the circuit tuned by the capacitor $c_{p}$ to a frequency $f=1 /\left(2 \pi \sqrt{L C_{p}}\right)$. If the length 1 of a shorted coaxial line is $\lambda / 4<I<\lambda / 2$, where $\lambda=c / f$, then it becomes $a$ capacitive element and cannot be tuned by $C_{p}$. Therefore, for $f=1.50 \mathrm{MHz}$ where $\lambda=200 \mathrm{~cm}$, the highest frequency we required, the coaxial line must be less than $50 \mathrm{~cm}$ long. The inductance of a coaxial line is proportional to In $(b / a)$, where $b$ is the inside diameter of 
the outer conductor and a... 1s the outside diameter of the inner conductor. In order that the field $\mathrm{H}_{1}$ be reasonably uniform throughout the sample, we need to make $b / a$ as close to unity as possible. However, $b-a$ must be at least $1 / 4 "$ in order that the annular cylinders of sample cast in paraffin can be physically strong enough to withstand handling.

The capacitor $C_{c}$ (shown in Fig. 4) is used to critically couple the tuned line to $50 \Omega$ for maximum power transmission. With a single adjustment of $\mathrm{C}_{c}$ the circuit remains critically coupled over a range of $10 \mathrm{MHz}$. The magnetic rf field $\mathrm{H}_{1}$ is maximum at the shorted end of the coaxial line. when the line is critically coupled, $\mathrm{H}_{1}$ has a magnitude of approximately 3 oe. The $Q$ of the tuned curcuit without any sample is 90 and 75 for coaxial lines with length 26 and $41 \mathrm{~cm}$ respectively. The $Q$ is fairly constant over the frequency range $100-150 \mathrm{mitz}$. With the sample inserted in the lines the $Q$ drops to 50 and 45 respectively. At $125 \mathrm{MHz}$ this represents a bandwidth of $2.5 \mathrm{MHz}$.

The coaxial line shown in Fig. 6, was constructed with 0.010" wall stainless steel tubing; stainless steel was used because of its low thermal conductivity. The outer and Inner conductors were made with 1 " o.d. and $\frac{1}{2} " 1 . d$. tubing respectively. The samples cast into annular cylinders could slide freely to the bottom of 

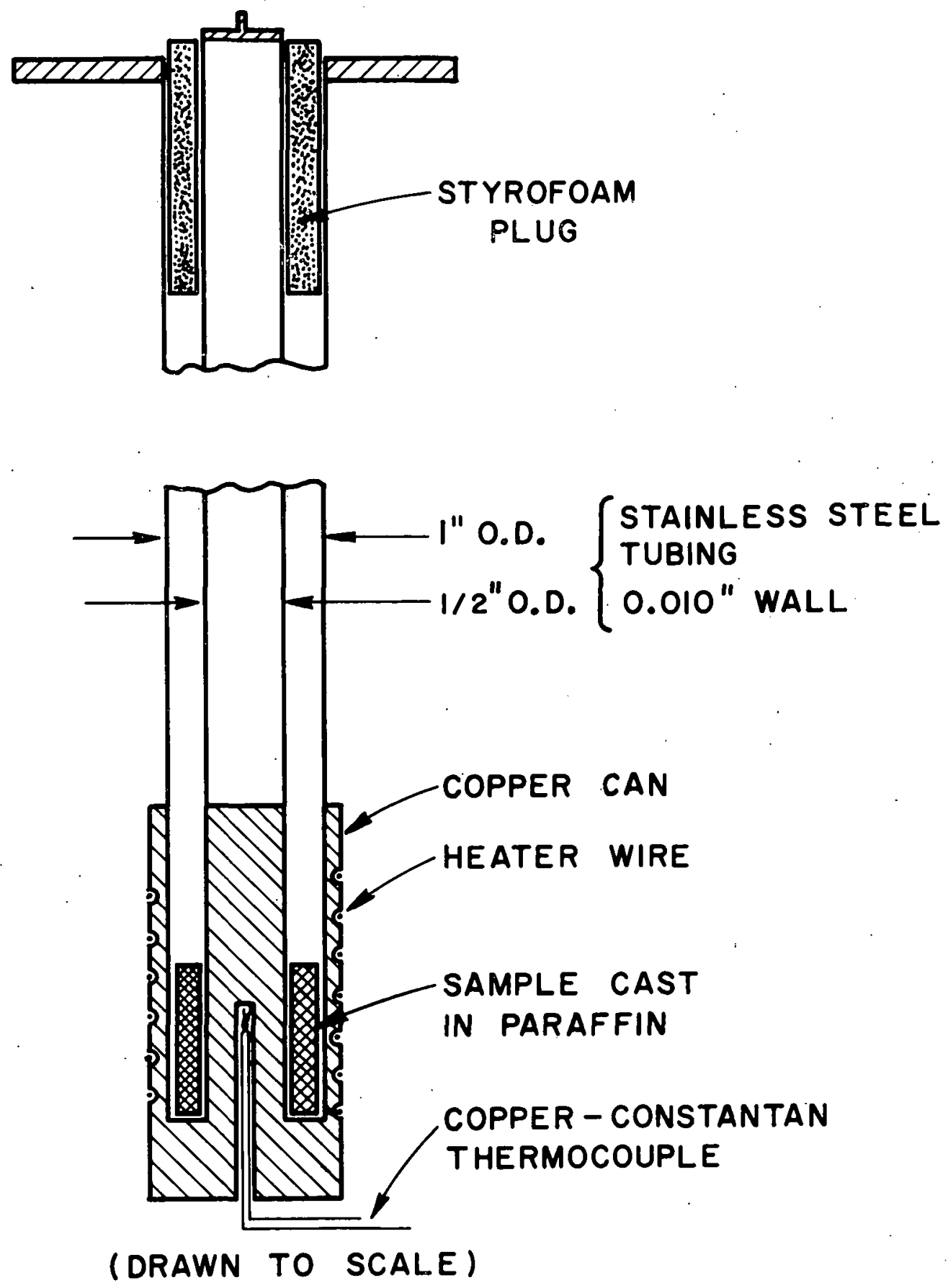

FIGURE 6. COAXIAL LINE 
this coaxial structure. We constructed lines 26 and $41 \mathrm{~cm}$ long for use in liquid nitrogen and liquid helium respectively.

To provide an isothermal environment for the sample, the bottom $6 \mathrm{~cm}$ of the line was a solid copper piece. A heater wire and thermocouple embedded in the copper provided continuous temperature control to within $\frac{1}{2}^{\circ} \mathrm{K}$. For work down to $77^{\circ} \mathrm{K}$ we surrounded the coaxial line with $1^{\prime \prime}$ of styrofoam insulation and placed it in a heavy copper can immersed in a liquid nitrogen bath. For work at lower temperatures we placed the line in a vacuum can and immersed it in a liquid helium bath. To study the effect of sudden displacements of the domain walls on the spin echoes, we need to apply short pulses of magnetic field to the sample. Fields of a few oersteds are usually required to displace domain walls a distance large compared with their width. The magnetic field pulse is to be applied during only one of the rf field pulses for reasons which we will explain later in this chapter. Since these may be separated by only $10 \mu \mathrm{sec}$, we require the magnetic field pulse to have a rise time less than 5 usec.

The magnetic field pulses were generated by discharging a capacitor through a coil in series with a gated diode. The diode gate was controlled by a variable time delay circuit triggered by the pulse train generator. 
The details of circuit design are given in appendix $c$. We were able. to produce $3 \mu \mathrm{sec}$ pulses with 1 koe maximum field in free space using an eleven turn coil, $4 \mathrm{~cm} \mathrm{1.d.} \mathrm{and} 4 \mathrm{~cm}$ long, wound with \#8 $\mathrm{cu}$ wire and a $3 \mu$ capacitor charged to $500 \mathrm{~V}$.

If the solid copper coaxial line were inserted in the coil, eddy currents would virtually eliminate the pulsed field at the sample position. To reduce these eddy current losses we constructed a special coaxial line. The solid copper bottom was split in half and then cemented back together with a thin mylar sheet sandwiched in between. This split was extended $8 \mathrm{~cm}$ up the stainless steel tubes by vertical slots cut with a fine saw. A field of 600 oe was measured inside this coaxial line in the plane of the mylar spaces. Away from this plane or at the bottom of the line where local eddy currents were larger, the field was half this value.

After some preliminary measurements it became clear that smaller fields were sufficient to displace the domain walls. By discharging a $1 \mu \mathrm{F}$ capacitor charged to $350 \mathrm{~V}$ we produced 270 oe pulses only 2 psec in duration at the sample. 


\section{Experimental Procedure}

When operating the spin echo spectrometer we used the following procedure. The rf oscillator was first adjusted to give two sharp, stable pulses 4-5 $\mu$ sec apart. These were monitored continuously with the $26 \mathrm{~dB}$ directional coupler. The recovery time of the oscillator was a problem. When consecutive pulses followed too closely, the amplitude of the second pulse varied slightly with the spacing. By careful adjustment of the oscillator, this effect could be confined to intervals less than $10 \mu$ sec., which was adequate for most relaxation measurements. When two pulses were separated by less than $2 \mu$ sec they broadened and tended to form a single pulse. Except for these restrictions; the pulse shapes and amplitudes were independent of each other.

Next we adjusted $c_{p}$ and $c_{c}$ for critical coupling to $50 \Omega$ at some anticipated NMR frequency $\nu_{0}$. We used a VSWR detector (Telonic Rho-tester TRB-3) in which the output of a balanced $r f$ bridge measures the reflected power from two impedance arms. We connected the coaxlal line and a standard $50 \Omega$ load to the two arms and applied rf power from a sweep generator (Kay 121-c Multi-Sweep)。 The output as a function of frequency was displayed directly on an oscilloscope. The output was zero when the two impedances were balanced. The frequency at critical coupling was easily measured with 
frequency markers from the sweep generator.

The most convenient way to set the rf power oscillator to $\nu$ was to first set the local oscillator to $\nu \pm 30 \mathrm{MHz}$. With 80-90 dB attenuation inserted to prevent saturation of the 1.f. amplifier, the frequency of the power oscillator was adjusted to maximize the rf pulses from the oscillator displayed on the oscilloscope. The attenuation was then removed and the spectrometer was ready for use with all elements tuned to frequency $\nu$.

If $\nu$ was chosen correctly the spin echo was clearly displayed on the oscilloscope. If not, the spectrometer could be slowly scanned in frequency by laboriously retuning the three frequency dependent elements. Since the bandwidth is approximately $2 \mathrm{MHz}$, the scanning was done in $2 \mathrm{MHz}$ steps. Once the echo was observed, its amplitude was maximized by cyclically adjusting the various tuning controls. If the temperature of the sample was changed slowly, it was easy to follow the NMIR frequency changes by retuning the spectrometer.

The frequency tuning of the spectrometer can be made simpler. We can construct a broadband balanced bridge if the coaxial line is connected directly to one port of the hybrid junction and is balanced by a varlable attenuator and phase shifter on the other port. This arrangement was not used since the sensitivity of the 
spectrometer was then reduced by more than a factor of 10 .

The shape and amplitude of the echo was sensitive to the $r$ excitation condition. In order to be able to compare different measurements we always adjusted the rf pulses to obtain a maximum echo amplitude. The rf pulses were narrowed to less than $0.4 \mu$ sec at which point their amplitude began to drop. We were unable to determine if the optimum $120^{\circ}$ rf pulse condition was obtained. Since the rf field is enhanced in a complicated way and is attenuated as it penetrates the sample, the individual nuclei will see a wide range of $\mathrm{rf}$ field strengths. The second $r f$ pulse was slightly wider than the first but the pulse widths were never in the ratio $1: 2$ required for a $90^{\circ}-180^{\circ}$ pulse train.

NMR frequency measurements were made by measuring the frequency of the local oscillator using a HewlettPackard 5345L counter and 5254B frequency converter. In this way we were measuring the frequency of the echo which may be slightly different from the Larmor frequency. 57 . Although the Zeeman line of the vin site is split by quadrupole interaction into 5 lines approximately $0.5 \mathrm{MHz}$ apart, we observed only a single echo maximum. The frequency distribution of the rf field pulses is the Fourler transform of the pulse shape. For $0.5 \mu \mathrm{sec}$ pulses the frequency distribution will be approximately $2 \mathrm{MHz}$ wide. For this reason the 
individual. Ines are not resolved. The width of the frequency distribution is given by

$$
\Delta \nu \approx 1 / \Delta \mathrm{T},
$$

where $\Delta T$ is the width of the pulse. The center frequency of the echo agreed with the frequency of the center line obtained from cw measurements。 58 Although the frequency was measured to a precision of $\pm 1 \mathrm{kHz}$, the accuracy was Iimited by the tuning of the spectrometer. We were able to achieve reproducible measurements with an accuracy of $\pm 100 \mathrm{kHz}$.

\section{Relaxation Time Measurements}

The output of the video detector as a function of time is shown in the sketch drawn below.

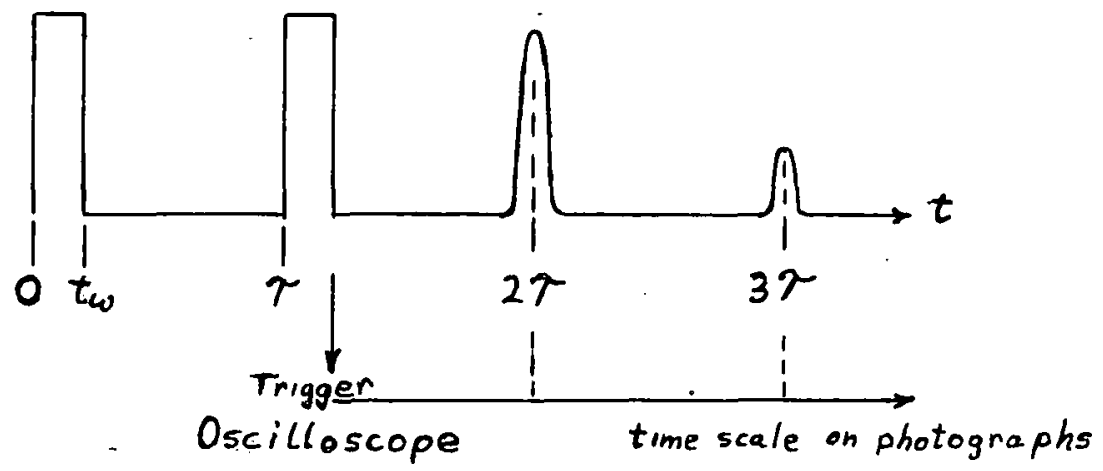

The first two pulses are the exciting rf field pulses from the oscillator which saturate the i.f.amplifier. The spin echoes appear at $t=2 \tau$ and $3 \tau_{0}$ Between sweeps of the pulse generator we allowed sufficient time, at least 1OT, $_{q}$ for the nuclear spins to return to 
thermal equilibrium. The oscilioscope was triggered at the end of the second rf puise so that only the spin echoes were displayed. By continuously varying the delay time $\tau$ between the two $r f$ pulses, multiple exposure photographs were taken directly from the oscilloscope display. Examples of these photographs are shown in Fig. 18 .

The equations for echo modulation by $H$. Abe et al..s which we have discussed in Chapter II, include phase factors and higher harmonic terms whose amplitude depend on the off-diagonal elements of the matrices $U_{I}$ and $U_{I I I^{\circ}}$ These matrix elements are sensitive to the rf pulse shape, so that the amplitude and symmetry of the spin echo envelope modulation depended on the oscillator adjustment. Nevertheless, the exponential decay of the spin echo amplitude should be independent of this. All relaxation measurements were taken from the envelope of the first echo with the rf pulses adjusted to maximize this echo.

The amplitude of the envelope is given by

$$
\begin{aligned}
& A(\tau)=\left\{c+b \exp \left(-2 \tau / T^{*}\right) \cos \left[\left(2 \pi / \tau_{m}\right) \tau+\delta\right]\right\} \\
& x \exp \left(-2 \tau / T_{2}\right)
\end{aligned}
$$

The amplitude of the envelope was measured from the photographs and then plotted on graph paper to obtain $\mathrm{T}_{2}$ directly as we expected. The values of $\mathrm{T}_{2}$ were 
insensitive to the exact adjustment of the rf pulse shape. The measurements of the envelope.modulation will be considered in the last chapter.

We measured $T_{1}$ by using a sequence of three $r f$ puises. The diagram below shows the pulse train that we first used to measure $T_{1}$ 。

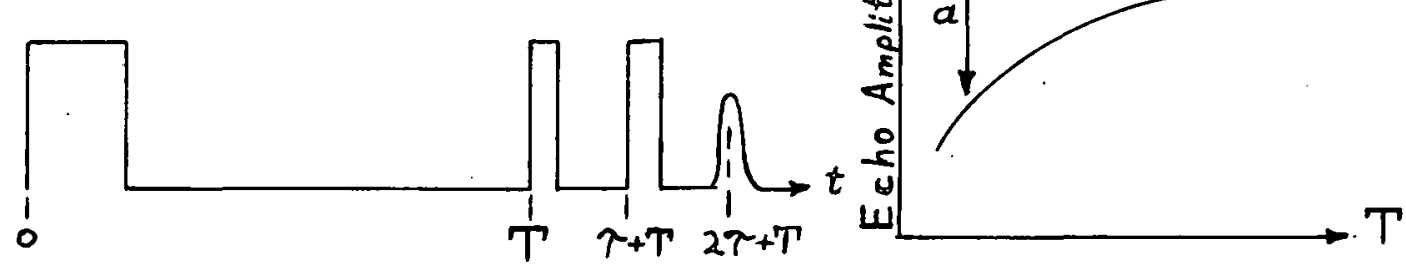

The initial pulse saturated the nuclear spin system producing an isotropic distribution of spins. After an interval $T$ we applied a normal pulse pair, producing an echo at $T+2{ }^{2}$. The amplitude of the echo was then proportional to the number of nuclear spins that had relaxed to thermal equilibrium during time $T$. With $\uparrow$ fixed, we varied $T$ to produce the envelope shown above. From a graph of a vs $T$, where a is the difference of the envelope amplitude and its maximum value, we measured $T_{1}$ directly。

Because of the difficulty in determining the base line from which a was measured, we used instead the 3 pulse pattern shown below for most of our work. Here the "stimulated echo" is detected. Since its amplitude is proportional to $\exp \left(-T / T_{q}\right)$, measurements of $\mathrm{T}_{1}$ were much simpler and more accurate. 


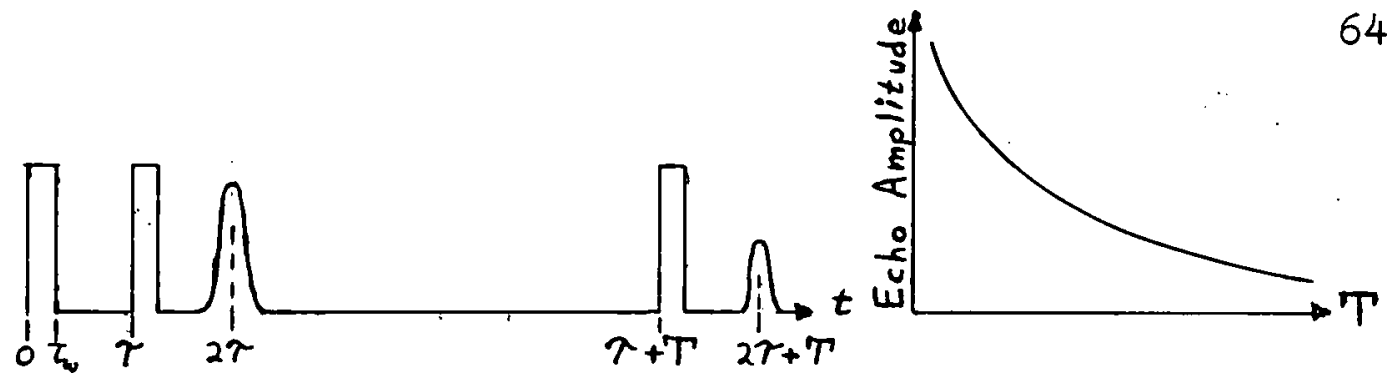

The oscilloscope sweep. Was triggered at the end of the second rf pulse but the trace intensity was suppressed until after the third rf pulse by applying a blanking voltage directly to the grid of the cathode ray tube (see appendix B). In this way only the "stimulated echo" was photographed. However, for Iong blanking times, uneven trace intensities made it.impossible to produce clear photographs. For these cases we used a boxcar integrator which amplifies and integrates a signal passing through an input gate. We centered the gate, $1 \mu$ sec wide, on the peak of the "stimulated echo" Then only this part of the output from the video detector was sampled by the integrator. The gate position was fixed relative to the third $r f$ pulse so it stayed on the peak of the echo as $T$ was varied. The output of the boxcar integrator was plotted on the $y$-axis of an $X Y$ recorder. On the $\mathrm{x}$-axis we applied a voltage proportional to $T$ obtained from inside the GR pulse generator. From the trace of the echo decay $T_{1}$ measurements. were made. This procedure was used for work below $77^{\circ} \mathrm{K}$ where $\mathrm{T}_{1}$ became very long. 
Sources of Spin Echo Signal

Our NMR study of the $\mathrm{Sb}$ site was very limited since the spin echo was barely visible: At $77^{\circ} \mathrm{K}$ the maximum signal to noise ratio was only $3: 1$ so only approximate frequency measurements could be made. The echo signal from the two Mn sites, however, was very strong and at $77^{\circ} \mathrm{K}$ had a maximum signal to noise ratio of $400: 9$. As a result we were able to study the effect of variation of rf power or external magnetic field on the echo。

To be able to interpret our data we need to understand the source of the spin echo signal. We expect the relaxation times of nuclei in the domains to be longer than the relaxation times of those in the domain walls. At low rf power the echo should be from the domain wall nuclei since wall motion gives these nuclei a larger enhancement factor and shields the domain nuclei from the If field. At higher power levels the domain wall signal will become saturated and $\mathrm{rf}$ field will penetrate into the domains. It is difficult to estimate what proportion of the echo signal comes from each source. Because of the attenuation of the rf field in the material, the nuclei in both the domain and domain wall will see a range of power levels.

The "stimulated echo" decay shows evidence of both domain and domain wall contributions to the spin echo. 
Below we show:a typical decay curve.

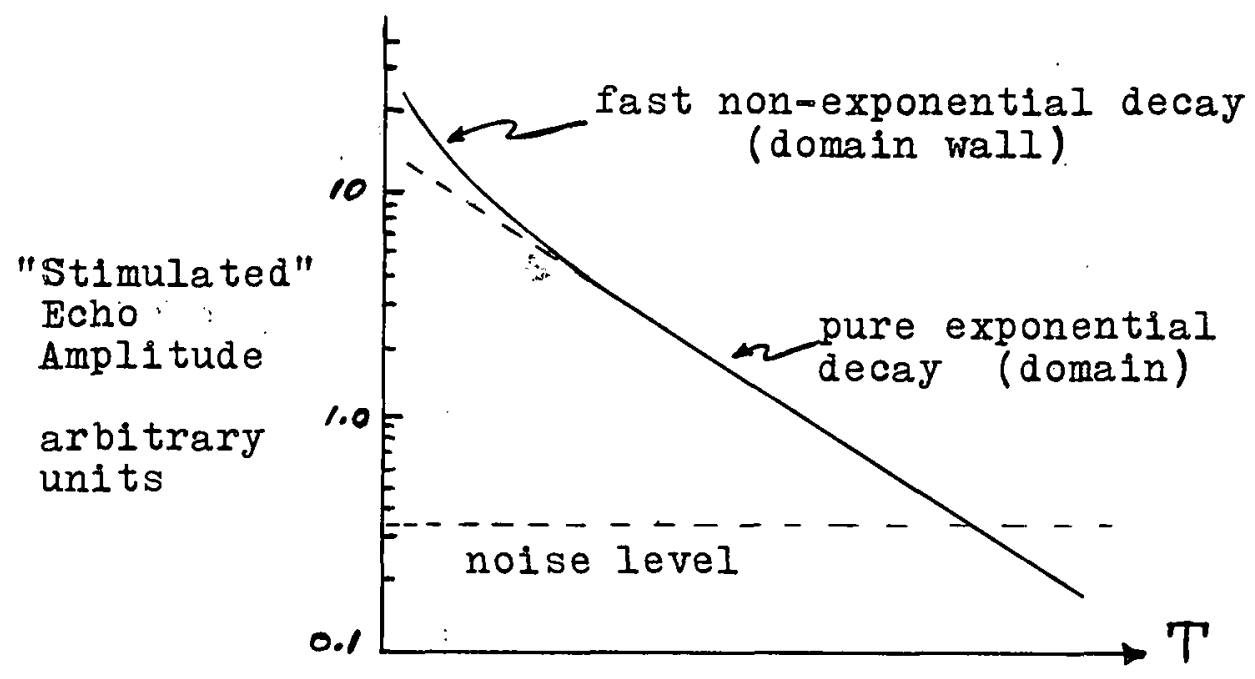

The faster, nonexponential decay probably comes from spins in the domain wall. The longer, exponential decay gives us the intrinsic relaxation time $T_{1}$ of the spins in the domain. To be sure to measure this value of $I_{1}$ we always set the gain of the 1.f. amplifier so that we could see the echo decay to the notse level. We performed a series of measurements to determine the source of the spin echo. First we measured $T_{1}$ and $T_{2}$ as function of power (see Fig. 8). As the power was increased the relaxation time lengthened rapidly to a maximum value. We interpreted this as evidence of rf field penetration into the domain.

Next we applied a homogeneous magnetic field $\mathrm{H}_{0^{\circ}}$ From magnetization measurements shown on page 11 we estimate that $180^{\circ}$ domain walls are eliminated when $\mathrm{H}_{0} \doteq 2 \mathrm{kOe}$ leaving the $90^{\circ}$ walls which persist up to $\mathrm{H}_{0} \doteq 5 \mathrm{kOe}$. This description is consistent with the 


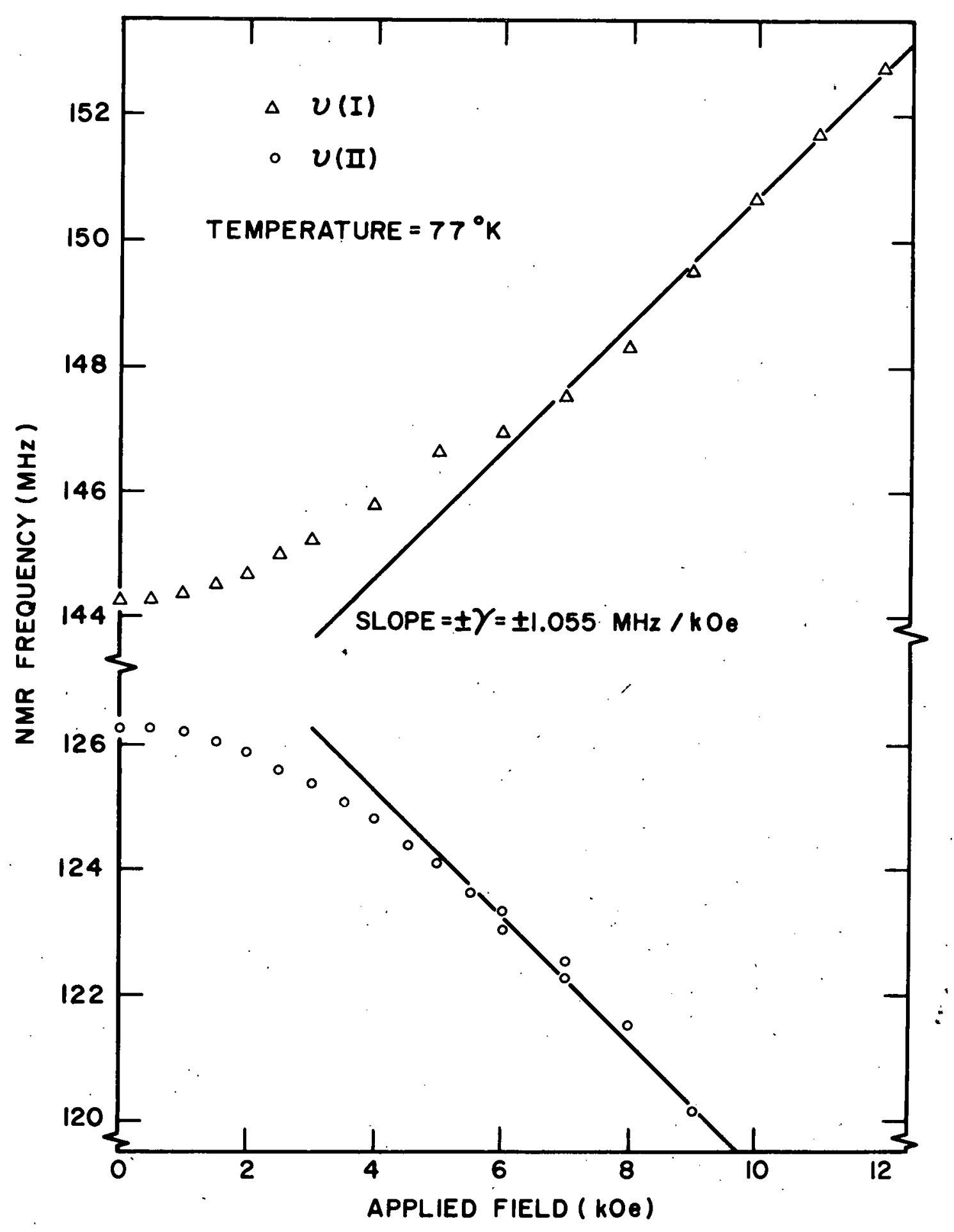

FIGURE 7. MAGNETIO FIELD DEPENDENCE OF THE NMR FREQUENCY 


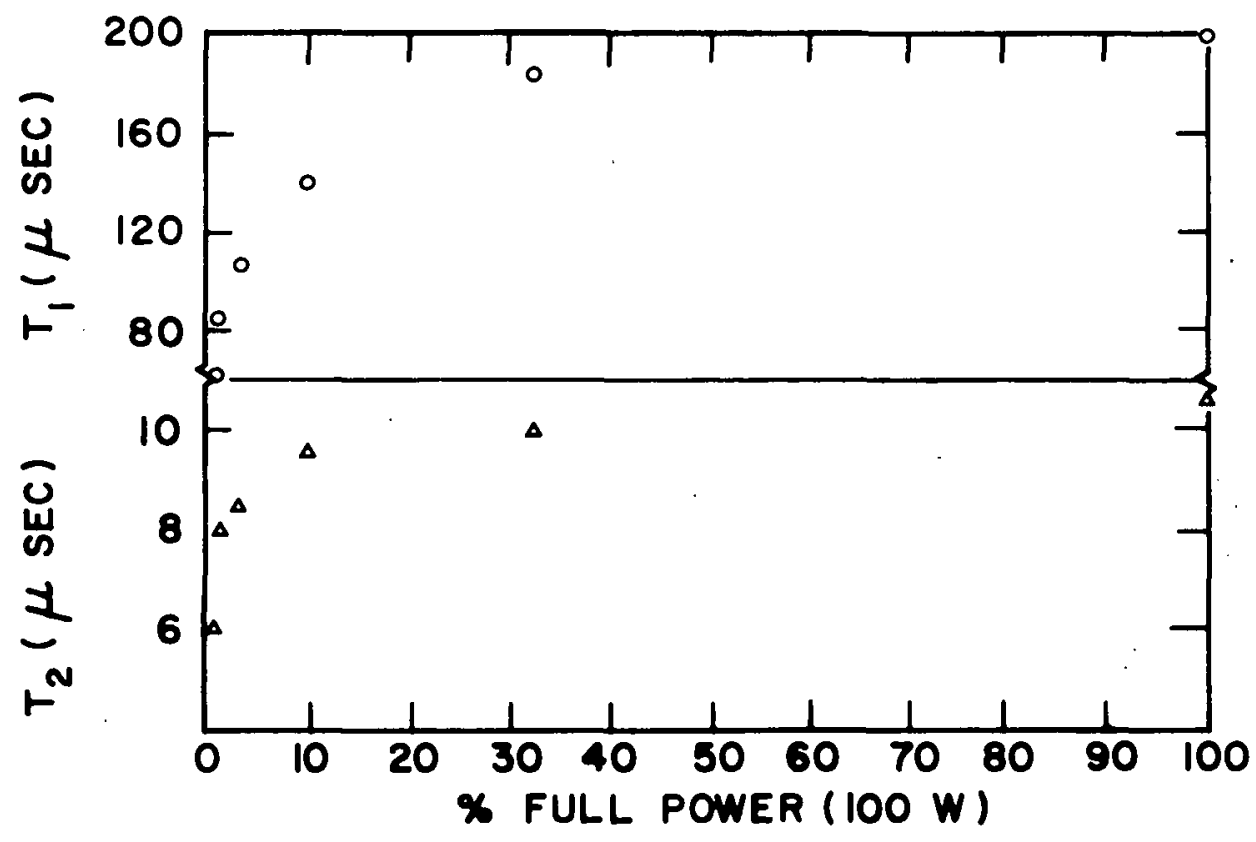

FIGURE 8. RF PONER DEPENDENCE OF $\mathrm{T}_{1}$ AND $\mathrm{T}_{2} \widehat{3} 77^{\circ} \mathrm{A}$

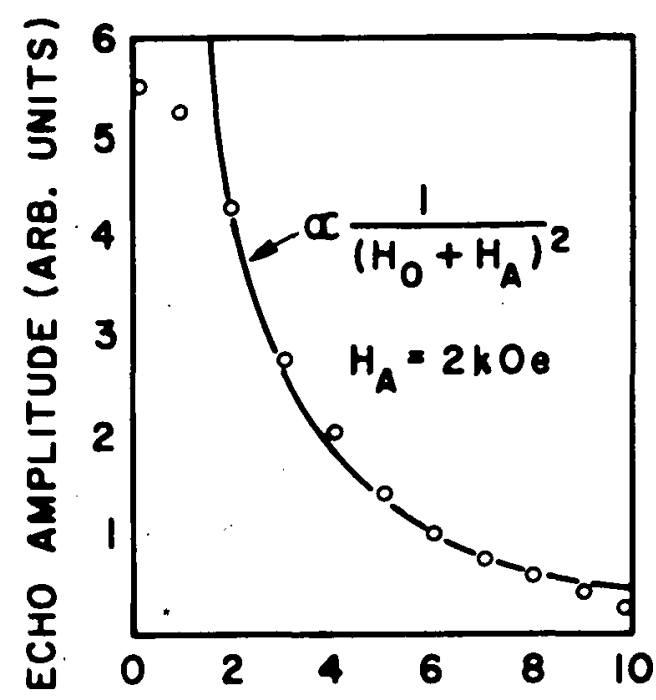

APPLIED FIELD $\mathrm{H}_{0}(\mathrm{kOO})$

FIGURE 9: MAGNETIC FIELD DEPENDENCE OF THE SPIN ECHO AMPIITUDE @ $77^{\circ} \mathrm{K}$ 
field dependence of the NMR frequency shown in Fig. 7. For small values of $\mathrm{H}_{0}$ the nuclei are almost completely shielded from the applied field as a result of demagnetizing fields and displacement of $180^{\circ}$ walls. Once the walls are swept away and the demagnetizing field reaches it.s maximum value N.M $_{S}$, changes in the local field are linear with $\mathrm{H}_{\mathrm{O}}$ : Above $\mathrm{H}_{\mathrm{O}}=5 \mathrm{kOe}$ the data is closely fitted by lines with slope $\pm \gamma$, the gyromagnetic ratio of the Mn nucleus.

A graph of the field dependence of the echo amplitude. 1s shown in Fig. 9. The nearly flat response at low fields again suggests domain wall displacement. Above $\mathrm{H}_{\mathrm{O}}=2 \mathrm{kOe}$ the echo amplitude data is fitted by a curve proportional to

$$
\eta^{2}=1 /\left(\mathrm{H}_{0}+\mathrm{H}_{\mathrm{A}}\right)^{2}
$$

with $\mathrm{H}_{\mathrm{A}} \approx 2 \mathrm{kOe}$. This is the behavior we expect for an echo signal from nuclei in the domains. We have calculated the domain enhancement

$$
\eta_{D}=1 /\left(H_{O}+H_{A}\right)
$$

There will be one factor of $\eta_{D}$ for the rf field enhancement to the nuclei and one factor of $\eta_{D}$ for the rf. signal from the precessing nuclear moment.

Our final test was to apply narrow pulses of magnetic field up to 500 oe before and during the second 
rf pulse. These are sufficient to displace the domain walls by distances much greater than their thickness. Thus no nuclei are in a domain wall for both $r f$ pulses and the echo is produced only from domain nuclei. Values of $\mathrm{T}_{1}$ were the same as those obtained in previous measurements. This confirms our assertion that the purely exponential decay is the intrinsic relaxation of the nuclear moments in the domain. 


\section{Chapter IV}

\section{Experimental Results}

\section{Introduction}

In this chapter we present the results of our NMR frequency and relaxation time measurements. At this time we omit discussion of phenomena associated with the reorientation of the easy axis of magnetization. This is of sufficient importance that we shall devote a separate chapter to 1 t.

\section{NMR Frequency Measurements}

In Fig. 10 we show the temperature dependence of the NMR frequency of the various nuclear sites. Details of the high and low temperature ranges will be given separately。 At high temperatures the spin echo amplitude rapidly decreased. We stopped making frequency measurements when the signal to noise ratio was about $3: 1$.

Since the spin echo from the Sb nucle1 was always small, only crude frequency measurements were made. The quadrupole splitting is so large that echoes were seen. over a 5-10 MHz interval. The frequency range indicated in Fig. 10 is the region over which echo amplitude maxima were obtained. Within this region we were not able to resolve the individual lines. At $77^{\circ} \mathrm{K}$ the echo from the other isotope $\mathrm{Sb}^{121}$. was observed at $250 \mathrm{MHz}$. Since 


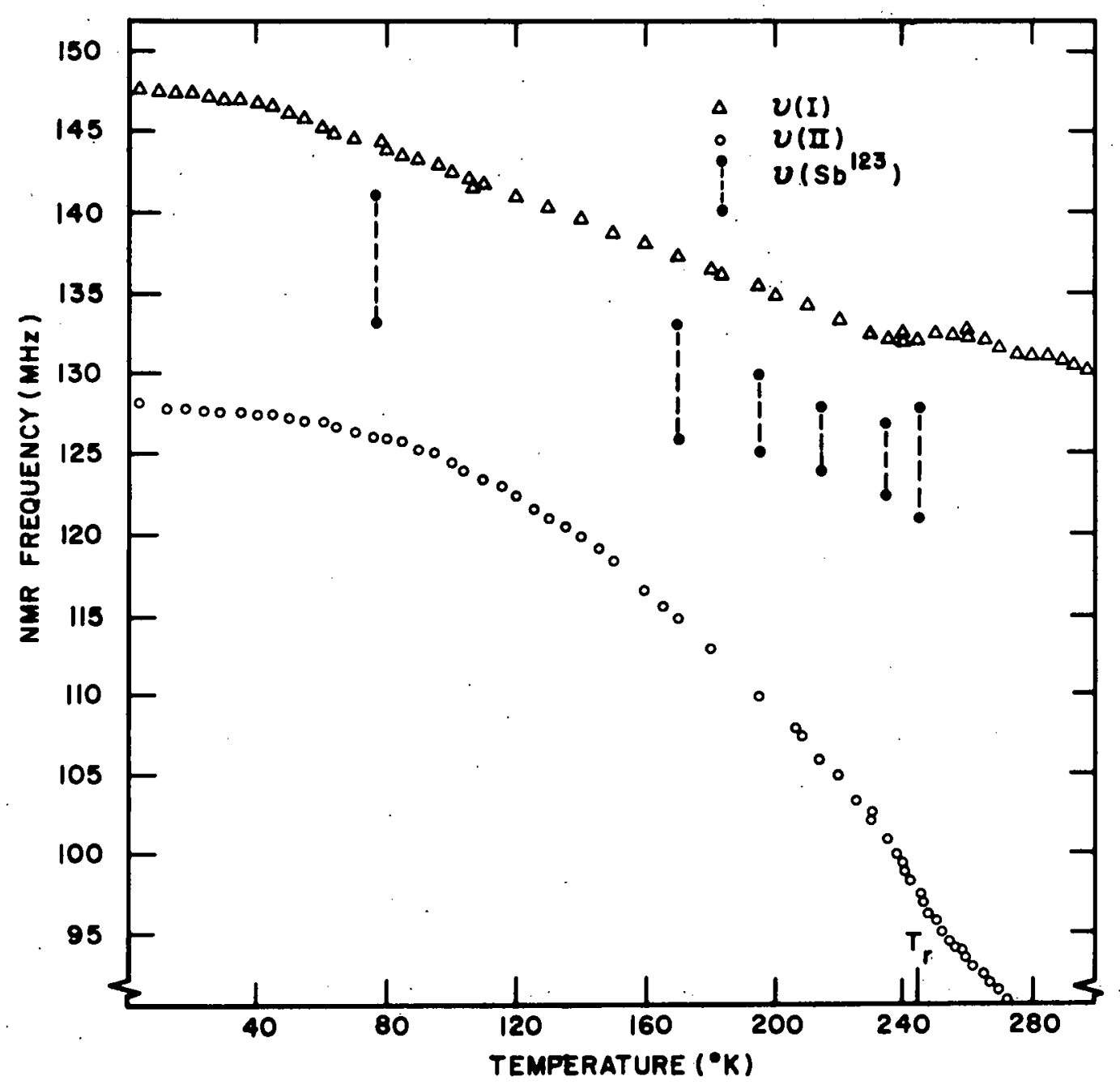

FIGURE 10. TW SERATURE D TENDINCE OF THE MRR FREQUENCY FOR DIFF RRENT NUCLEAR SITES IN $\mathrm{Mn}_{2} \mathrm{Sb}$ 


$$
\frac{\nu\left(s b^{121}\right)}{\nu\left(s b^{123}\right)}=\frac{\gamma\left(s b^{121}\right)}{\gamma\left(s b^{123}\right)}
$$

we know that these echoes come from the $\mathrm{Sb}$ atoms.

In the case of Mn we have one naturally occurring isotope $\mathrm{Mn}^{55}$ and two inequivalent lattice sites $\mathrm{Mn}$ (I) and Mn(II). The temperature dependence of the NMR frequency of the two sites is strikingly different. At low temperatures $\nu(I)$ decreases with increasing temperaw tures faster than $\nu$ (II). However, above $60^{\circ} \mathrm{K}$ the decrease in $\nu(I)$ is neariy linear in temperature while now $\nu$ (II) decreases at a much faster rate.

The temperature dependence of $\nu(\mathrm{Sb})$ appears to follow $\nu(I)$. We recall that from the spin density measurement.s discussed in Chapter II, Alperin et al. 8 suggested that the ordering of the Mn(II) sites takes place via coupling to the Sb atoms. One would then expect that the temperature dependence of the magnetic field at these two sites should be similar. Instead we observe that the temperature dependence of the fleld at the $\operatorname{Mn}(I)$ and $\mathrm{Sb}$ sites are similar。

\section{Frequency Identification of the Mn Sites}

In Fig. 10 we have identified the frequencies as $\nu(I)$ and $\nu(I I)$. These are associated with the lattice sites $M(I)$ and $M$ (II) respectively。 We will now justify this. 
Assuming that the hyperfine interaction is constant, then $\nu$, where $\nu=\omega_{0} / 2 \pi$, is proportional to the local magnetization $\sigma(T) \propto\left\langle S_{Z}\right\rangle$. We normalize the frequency measurements by. setting

$$
\zeta=\nu(T) / \nu(0)
$$

Then the total magnetization of $\mathrm{Mn}_{2} \mathrm{Sb}$ will be

$$
M(T) h m r=\left|\mu_{I I} \zeta_{I I}(T)-\mu_{I} \zeta_{I}(T)\right| 。
$$

We expect this to have a temperature dependence similiar to that of the magnetization measurements shown in Fig. 2。 When the higher frequency is identified as $\mathcal{\nu}(I)$, then $M(T)_{n m r}$ has approximately this temperature dependence as is shown in Fig. 12(c)\%. If we were to identify the higher frequency as $\nu$ (II) then $M(T)$ is constant at low temperatures and begins to increase at $200^{\circ} \mathrm{K}$. This behavior is so contrary to the magnetization measurements that we are confident that the first assignment is correct。

\section{Direction of the Hyperfine Field}

In Fig。 7 we show $\nu(I)$ and $\nu(I I)$ as a function of an applied field $\mathrm{H}_{0}{ }^{\circ}$ When $\mathrm{H}_{0}>5 \mathrm{koe}$ the magnetization of the sample is saturated and the effective field at the nucleus becomes

$$
\overrightarrow{\mathrm{H}}_{\mathrm{e}}(i)=\overrightarrow{\mathrm{H}}_{\mathrm{hf}}(i)+\overrightarrow{\mathrm{H}}_{0}+\text { const。 } \quad(i=I \text { or II })
$$


The slope of $\nu(I I)$ vs. $H_{0}$ is $-\gamma_{0}$ since $\mu_{I I}>\mu_{I}$, the magnetization of the Mn(II) sublattice defines the direction of magnetization for the whole sample and $\vec{\mu}_{\text {II }}$ is parallel to $\overrightarrow{\mathrm{H}}_{0}$. Since $\mathrm{H}_{\mathrm{e}}$ (II) decreases as $\mathrm{H}_{0}$ increases we must have $\overrightarrow{\mathrm{H}}_{\mathrm{hf}}$ (II) antiparallel to $\overrightarrow{\mathrm{H}}_{0}$, $1 . e .$, $\mathrm{H}_{\text {hf }}$ is negative. It follows that the slope of $\nu$ (I) is $+\gamma$ since $\vec{\mu}_{I}$ is antiparallel to $\overrightarrow{\mathrm{H}}_{0}$. The hyperfine interaction is negative in ferromagnetic metals such as $\mathrm{Fe}, \mathrm{Ni}$, and Co so we expected the same result in $\mathrm{Mn}_{2} \mathrm{Sb}$.

\section{Sublattice Magnetization}

For most magnetic systems, the magnetization at low temperatures is successfully described by spin wave theory and has the form

$$
m(T)=m(0)\left[1-a T^{3 / 2}-b T^{5 / 2}-c T^{7 / 2}-\ldots\right] \text {. }
$$

The higher order terms are obscured by the spin wave spin wave scattering and single particle excitation contributions which have a $\mathrm{T}^{4}$ dependence. For a locallzed ionic model it is easy to show that the low level excitationsare spin waves. From the constants $\underline{a}_{,} \underline{b}_{\text {}}$ and $\subseteq$ the sublattice coupling in a ferrimagnet can be determined. To do so the magnetization data must be accurate to one part in $10^{5}$ or $10^{6}$. Since our frequency measurements were only accurate to one part in $1.0^{3}$, this was not attempted. 
In magnetic metals there are additional complications and the theory is not as fully developed.59 coupling can also'occur via the itinerant electrons so there will be a mixture of spin wave and single electron excitation. However, measurements of ferromagnetic metals such as $\mathrm{Fe}, \mathrm{Ni}$, and $\mathrm{Co}$ all show that $\mathrm{T}^{3 / 2}$ terms dominate at low temperatures.60

In Fig. 11 we plot $\nu(I)$ and $\nu$ (II) as a function of $\mathrm{T}^{3 / 2}$. The two have strikingly different behavior as $\nu(I)$ appears to fit a $\mathrm{T} 3 / 2$ law out to $120^{\circ} \mathrm{K}$ while $\nu$ (II) drops off with a higher power of temperature. The data are not sufficiently accurate to compare their temperature dependence below $60^{\circ} \mathrm{K}$.

The $T^{3 / 2}$. fit of $\nu(I)$ to such a high temperature may be spurious and not the result of spin wave excitations. At higher temperatures we expect that the higher power terms will become dominant and that $\nu(T)$ will decrease faster than $\mathrm{T} 3 / 2$. However, $\nu(I)$ decreases approximately linearly with increasing temperature, and we see the data deviating from the $\mathrm{T}^{3 / 2}$ line in the "wrong" direction. We can not suggest any mechanism that would produce such an unusual temperature dependence.

This leads us to question whether $\nu(T)$ is proportional to the sublattice magnetization as we have assumed. In Fig. 12 we show the temperature dependence of the sublattice and total magnetization 


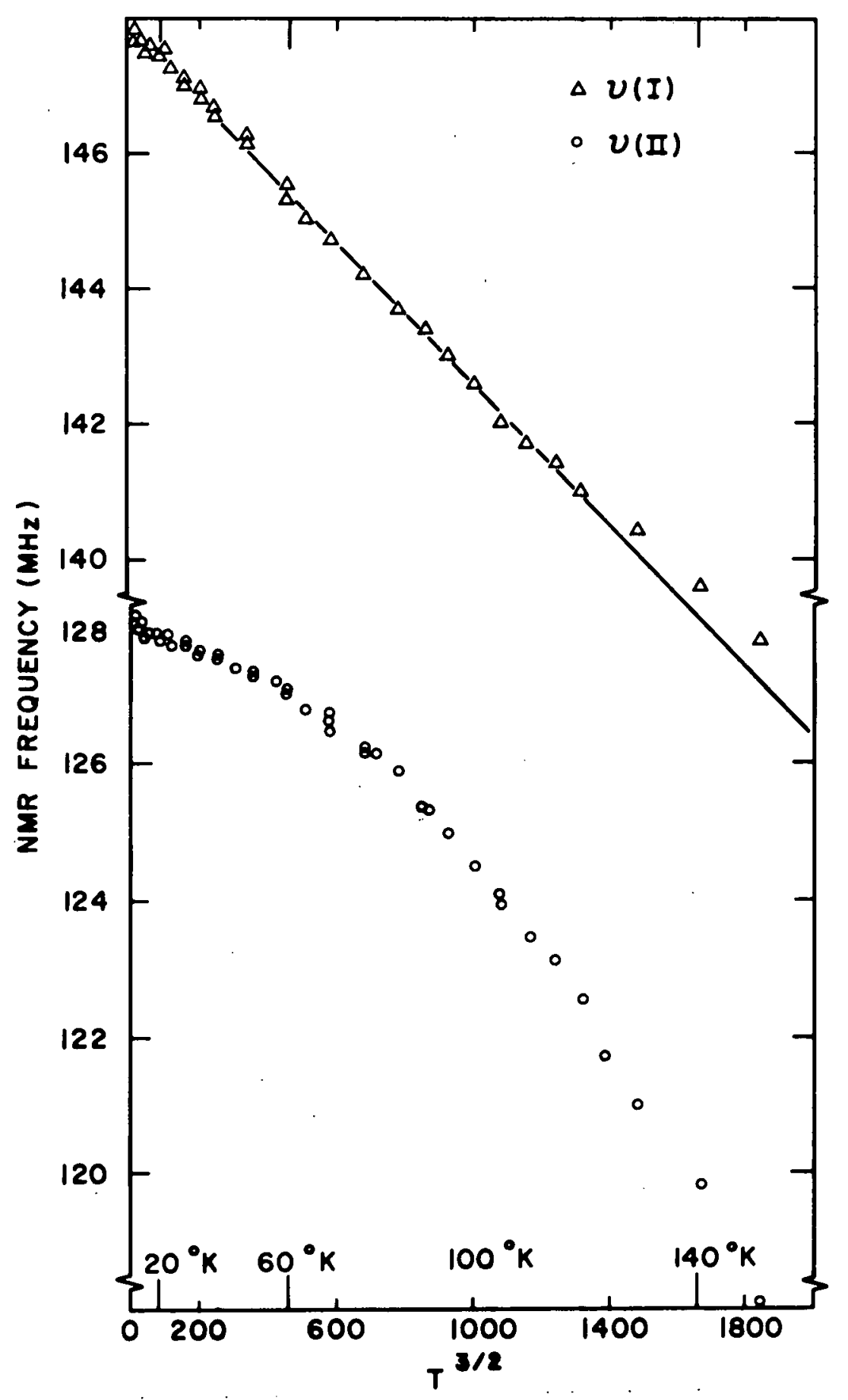

FIGIRE 11. TRR FREQJEICY OF THE UIn SITSS AS A 
normalized to unity at $T=0$. Our frequency data are compared with molecular field calculations of $\sigma_{I}(\mathrm{~T})$ and $\sigma_{I I}(T)$ using the constants fitted to magnetization data by Guillaud. 19 Although the molecular field theory cannot account for the temperature dependence of the magnetization at low temperatures, it gives a good description -- to within $1 \%$ - of the high temperature magnetization data.

In Fig. 12 (c) we compare the total magnetizations $M(T)$. We define the calculated and experimental magnetizations as follows:

$$
\begin{aligned}
\mathrm{M}(\mathrm{T})_{\text {calc }} & =\frac{\mu_{I I} \sigma_{I I}(\mathrm{~T})-\mu_{I} \sigma_{I}(\mathrm{~T})}{\mu_{I I}-\mu_{I}} \\
\mathrm{M}(\mathrm{T})_{\mathrm{nm}} & =\frac{\mu_{I I} \zeta_{I I}(\mathrm{~T})-\mu_{I} \zeta_{I}(\mathrm{~T})}{\mu_{\mathrm{II}}-\mu_{I} .}
\end{aligned}
$$

Qualitatively, $\mathrm{M}(\mathrm{T})_{\mathrm{nmr}}$ has the same temperature dependence as $M(T)$ calc. The two fit well up to $120^{\circ} \mathrm{K}$. At higher temperatures $M(T)_{n m r}$ falls rapialy below $M(T)$ calc. At $240^{\circ} \mathrm{K}$, the error is over $10 \%$

In Fig. $12(\mathrm{~b})$ we have nearly the same situation. The shape of $\zeta_{\text {II }}(\mathrm{T})$ is qualitatively similar to $\sigma_{\text {II }}(\mathrm{T})$. The two curves begin to deviate at $50^{\circ} \mathrm{K}$. The comparison of $\sigma_{I}(T)$ and $\zeta_{I}(T)$ in Fig. $12(\mathrm{c})$ is different. The two curves do not have the same shape. Above $50^{\circ} \mathrm{K}, \zeta_{\mathrm{I}}(\mathrm{T})$ is linear in temperature, while $\sigma_{\mathrm{I}}(\mathrm{T})$ 


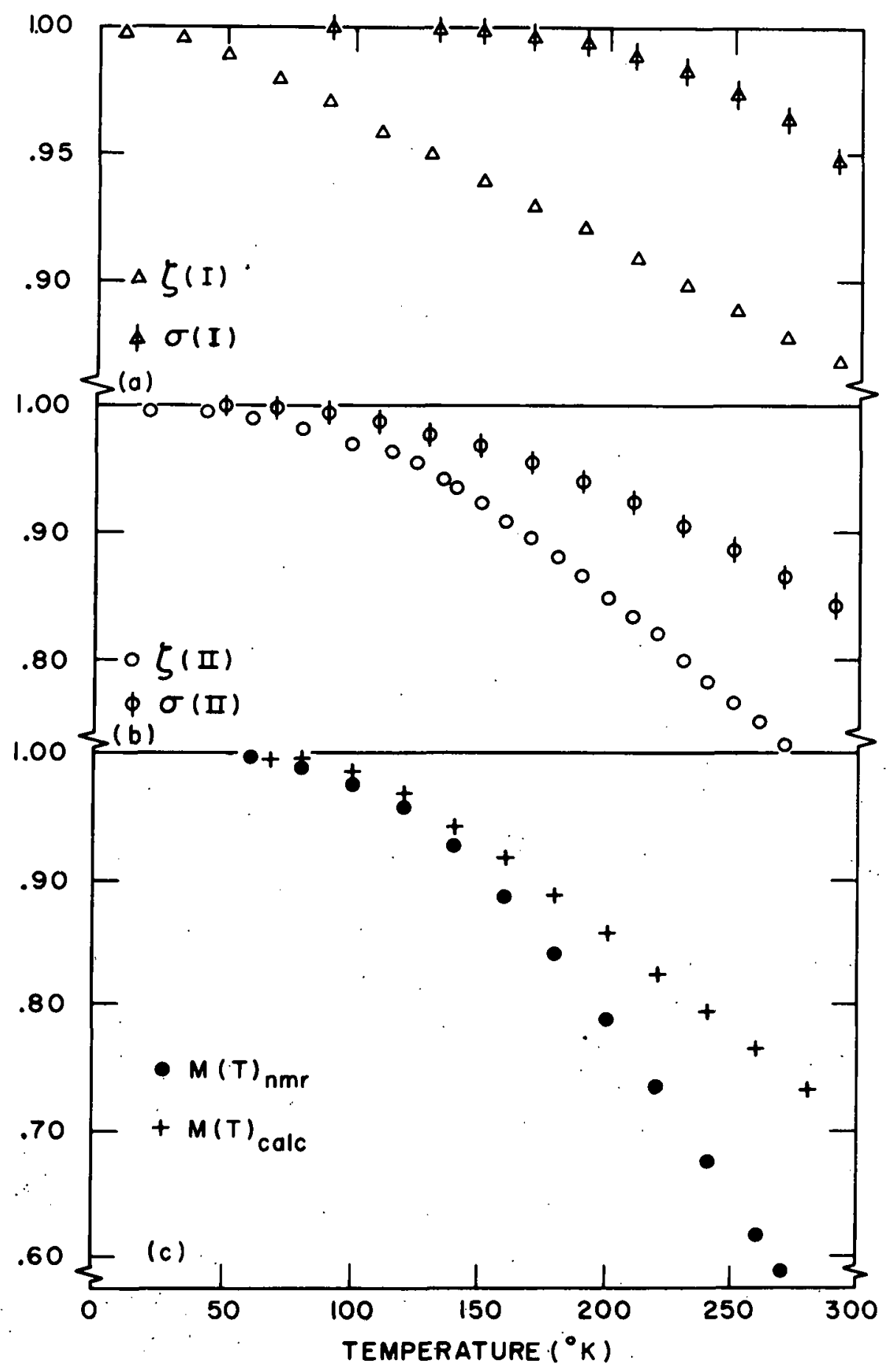

FIGURE:12. SUBLATTIOE MAGNETIZATION: A CCMPARI SON OF THEORITIOAL OALCULATIONS HAND NMR FREQUSTCY WIEA JURERENTS 
is nearly constant out to $150^{\circ} \mathrm{K}$. At high temperatures the slope of $\sigma_{I}(I)$ increases rapidly and becomes greater than the slope of $\zeta_{I}(T)$. Thus $\zeta_{I}(T)$ does not appear to describe even qualitatively the temperature dependence of the local magnetization of the $\operatorname{Mn}(I)$ lattice site. This difficulty may be caused by a temperature dependence of the hyperfine interaction which we have assumed is constant. Let us now assume that it can be written in the form

$$
A(T, a, c, \ldots)=A_{0}+A_{1} T^{3 / 2}+A_{2}(\underline{a})+A_{3}(\underline{c})+\cdots
$$

where a and $\underline{c}$ are the lattice constants. We have already considered the contribution from thermal fluctuation and found that $\left(\mathrm{A}_{1} \mathrm{~T}^{3 / 2}\right) / \mathrm{A}_{0}$ was less than $1 \%$ at $200^{\circ} \mathrm{K}$ for $\mathrm{Mn}^{++}$ in an insulator. We assume that for $\mathrm{Mn}$ in $\mathrm{Mn}_{2} \mathrm{Sb}$ the contribution is not appreciably larger.

The terms $A_{2}(\underline{a})$ and $A_{3}(\underline{c})$ represent the contributions to the hyperfine interaction due to the thermal expansion of the lattice. The lattice parameters $\underline{a}$ and $\underline{c}$ both increase monotonically with increasing temperature except at the F-AF and F-I-AF phase transitions, where they change discontinuously. We can approximate the magnitude of $\mathrm{A}_{2}(\mathrm{a})$ and $\mathrm{A}_{3}(\underline{c})$ in the following way. From the frequency measurements $\nu$ (I) and $\nu$ (II) in $\mathrm{Mn} \mathrm{N}_{2} \mathrm{Sb}$ and $\mathrm{Mn}{ }_{1.97^{\mathrm{Cr}}} \mathrm{r}_{.03^{\mathrm{Sb}}}$ (see Fig. 20), we obtain the dependence of the hyperfine interaction on the or concentration. 
Likewise from the lattice X-ray measurements of Darnell et al. ${ }^{13}$ we obtain the magnitude of a and $\underline{c}$ as a function of the Cr concentration. Combining these two results we can estimate the dependence of the hyperfine coupling due to changes in a and $\underline{c}$. The thermal expansion of the lattice parameters from $0^{\circ}$ to $200^{\circ} \mathrm{K}$ is $\Delta \underline{c} \approx 0.004 \AA$ and $\Delta \underline{a} \approx 0.015 \AA$. Assuming that $\nu=\nu(\underline{c})$, then $\Delta \nu(I) / \Delta \underline{c} \approx$ $160 \mathrm{MHz} / \AA$ and $\Delta \nu($ II $) / \Delta \underline{\underline{c}} \approx 120 \mathrm{MHz} / \AA$ and we will have $\Delta \nu(I)=0.6 \mathrm{MHz}$ and $\Delta \nu(I I)=0.5 \mathrm{MHz}$. On the other hand if we assume $\nu=\nu(\underline{a})$, then $\Delta \nu(I) / \Delta \underline{a}=23 \mathrm{MHz} / \mathrm{R}$ and $\Delta \nu(I I) / \Delta \underline{a}=17 \mathrm{MHz} / \AA$ and we will have $\Delta \nu(I)=0.4 \mathrm{MHz}$ and $\Delta \nu(I I)=0.3 \mathrm{MHz}$. We have no way of determinimg the exact functional dependence of the hyperfine coupling on a and $c$. However, it is clear that its contribution cannot cause a change in the hyperfine interaction by more than 1\%. This still falls short of accounting for this curious behavior of our data.

It is possible that the electronic state of the two Mn sites is strongly temperature dependent. The studies on $\mathrm{Mn}^{++}$which we have mentioned are for ionic crystals.o. .The results of these studies may not apply to $\mathrm{Mn}_{2} \mathrm{Sb}$ which has predominantly covalent bonding.

Nuclear Relaxation Times $\underline{T}_{1}$ and $\underline{T}_{2}$

All of our relaxation time data is shown in Fig. 13. The accuracy of the data is about $4-8 \%$ depending on the 


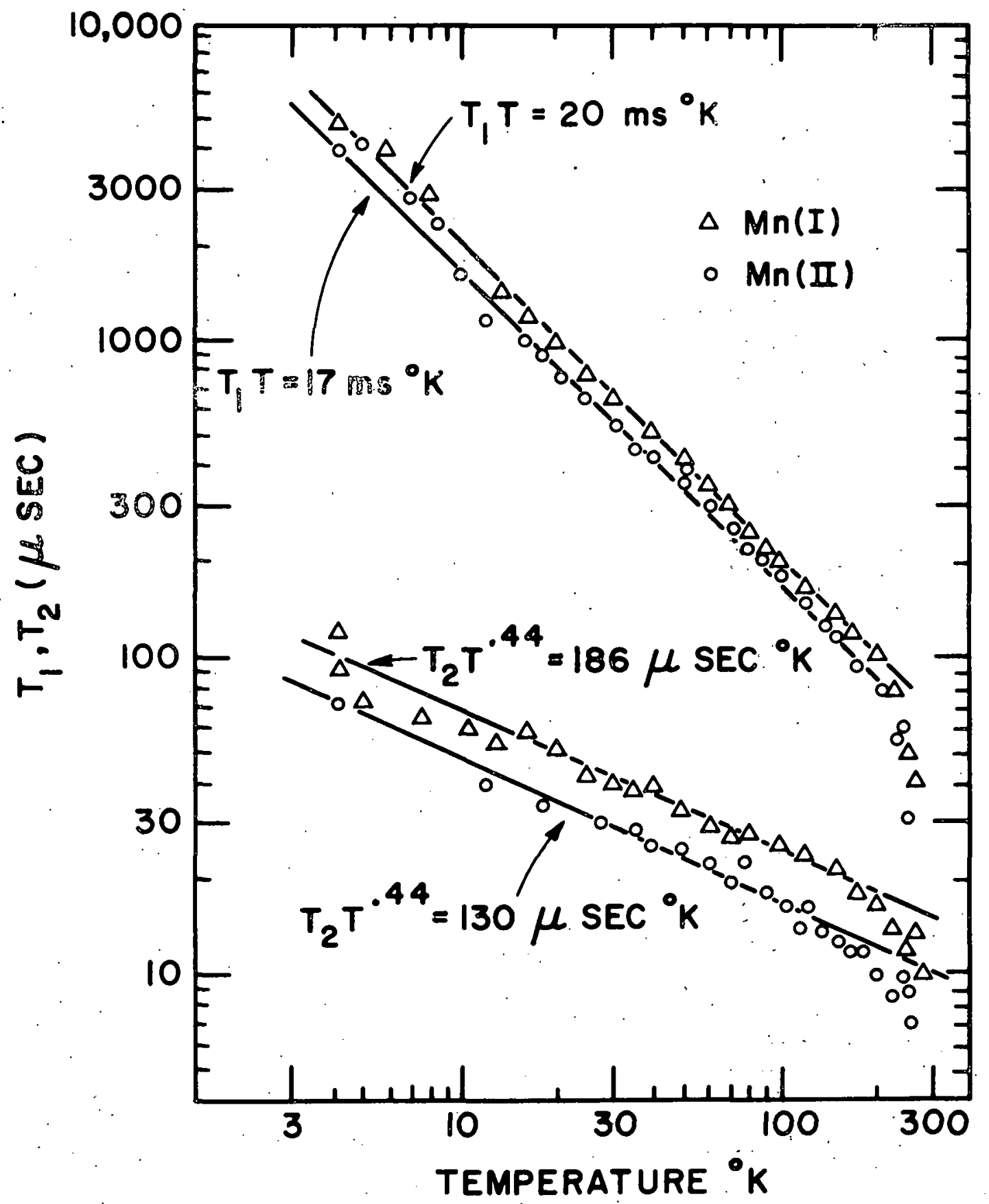

FIGURT 13. TEMP ERATURE DEPENDENCE OF $T_{1}$ AND $T_{2}$ 
amplitude of the echo. The temperatures are accurate to $\frac{1}{2}^{\circ} \mathrm{K}$ except for the measurements between 4.2 and $10^{\circ} \mathrm{K}$ where difficulties with the thermocouple may have introduced errors up to $1^{\circ} \mathrm{K}$. The lines drawn through the data are not calculated least square fits. For $T_{1}$ we were looking for the behavior $T_{1} T=$ constant and we use the line, drawn by eye, to how how closely the data follows a $\mathrm{T}^{-1}$ power. In the case of $\mathrm{T}_{2}$ no exact power law was expected. The line is drawn by eye only to suggest a possible fit. In contrast to the NIMR frequency data, the relaxation times of the two sublattices are remarkably similar. The sublattice with the larger moment has shorter relaxation times since the nuclear interactions are stronger.

The $T_{1}$ data are for the domain nuclei only. The constant $T_{1} T$ is $20 \mathrm{msec}^{\circ} \mathrm{K}$ and $17 \mathrm{msec}{ }^{\circ} \mathrm{K}$ for $\mathrm{Hn}(I)$ and Mn(II) respectively. This is an extremely short relaxation time. It is about $\frac{1}{4}$ of $T_{1} T$ for $C o$ which was the smallest of the ferromagnetic metals. The magnitude of the nuclear moment for $\mathrm{Mn}$ is nearly the same as for co. All of the discussion in chapter II on the difficulty of accounting for the short relaxation times in co applies for $\mathrm{Mn}$ in $\mathrm{Mn}_{2} \mathrm{Sb}$ too.

A surprising feature is the sharp break in the $T_{1}$ curve at $200^{\circ} \mathrm{K}$. The slope of the data approaches $\mathrm{T}^{-7}$. This suggests a quadrupole relaxation which is 
proportional to $T^{-7}$ for $T<0.02 \theta_{D}$, where $\theta_{D}$ is the Debye temperature. In $\mathrm{Mn}_{2} \mathrm{Sb} 200^{\circ} \mathrm{K}=0.3$ so this explanation is not very likely. There is no indication of anomalous behavior of $T_{1}$ about the reorientation temperature $\mathbb{T}_{\mathrm{r}}$.

In the $\mathbb{T}_{2}$ measurements no attempt was made to separate domain and domain wall contributions. We made measurements with maximum rf power so there was substantial penetration of the rf field into the domains.

Our values of $T_{2}$ are very close to those of Co measured by Weger et al. 49 The transverse relaxation is given by

$$
1 / T_{2}=1 / T_{2}^{\prime}+1 / T_{1},
$$

where $T_{2}^{\prime}$ is the spin-spin relaxation time. For Co and $M n$ in $\mathrm{Mn}_{2} \mathrm{Sb}, \mathbb{T}_{1} \gg T_{2}$ so that spin-spin interations are the predominant relaxation mechanism. According to the calculations and measurements of $\mathrm{T}_{2}$ in Co by $\mathrm{N}$. Kaplan et al. 61 , the nuclear dipole interaction, which is temperature independent, is the dominant contribution. It seems likely that this is also true for $\mathbb{M n}$ in $\mathrm{Mn}_{2} \mathrm{Sb}$. 


\section{Chapter V}

Magnetization Reorientation

\section{Introduction}

In this ohapter we will be concerned with the reorientation of the easy axis of magnetization, a phenomenon which occurs in a number of ferrimagnetic materials. We will first discuss measurements of the anisotropy energy in $\mathrm{Mn}_{2-\mathrm{x}} \mathrm{Cr}_{\mathrm{X}} \mathrm{Sb}$. Then we will outline recent work by Horner and Varma which predicts the existence of two second-order phase transitions in $\mathrm{Mn}_{2} \mathrm{Sb}$. We will conclude with a description of our measurements and discuss their connection with this theory. A preliminary report of these measurements has already been published. 62

\section{Anisotropy}

The anisotropy energy in $\mathrm{Mn}_{2-x} \mathrm{Cr}_{\mathrm{x}} \mathrm{Sb}$ has been studied by H. S. Jarrett et al. ${ }^{63}$ and F. Jo Darnell e.t al. ${ }^{13}$ at dupont using standard torque measurements. Since the crystal lattice has uniaxial symmetry, the data are fitted to a series expansion with even power terms of the form

$$
E_{A}=K_{1} \sin ^{2} \theta+K_{2} \sin ^{4} \theta+\cdots
$$

where $\theta$ is the angle between the direction of 


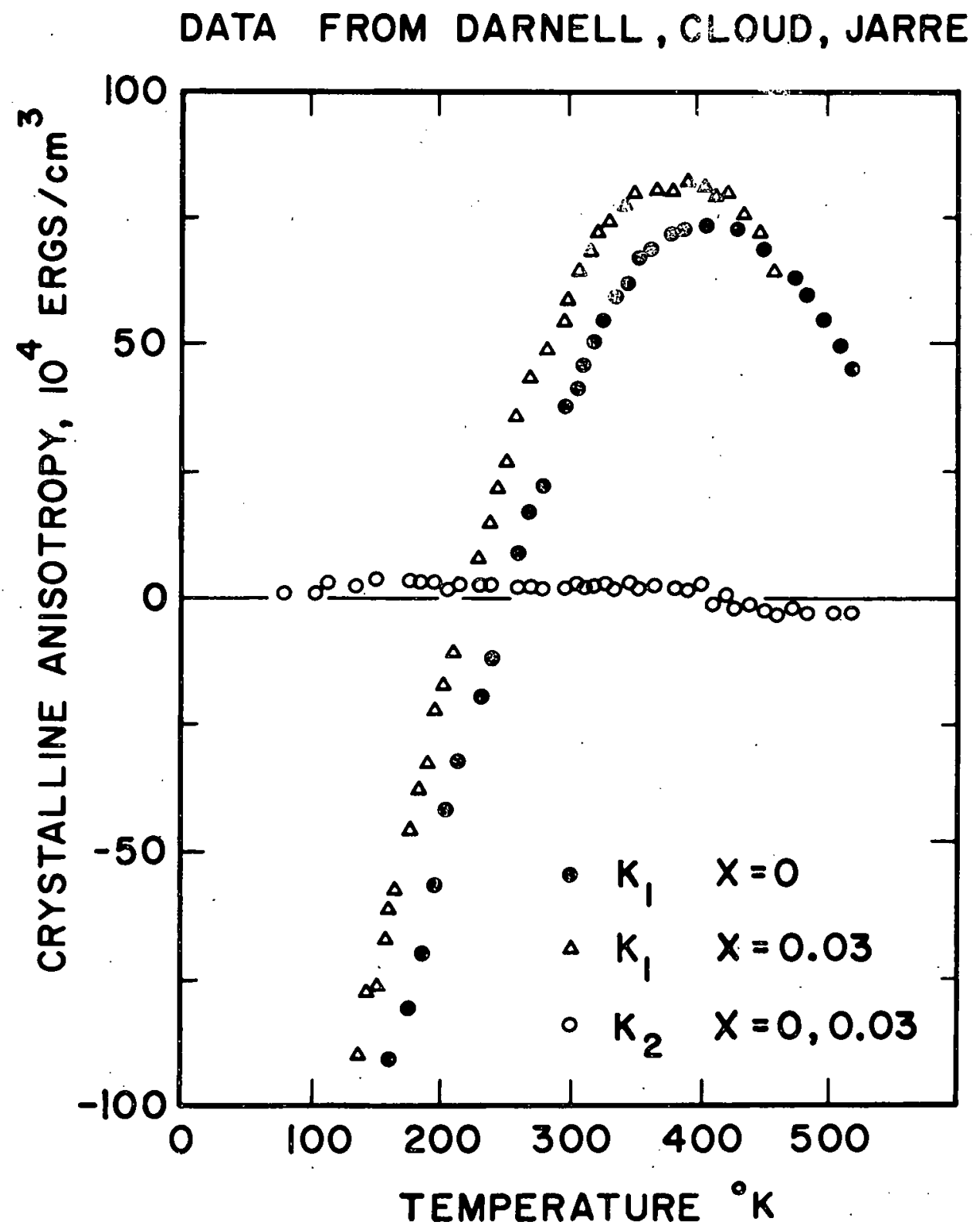

FIGURE 14. TEMPERATURE DEPENDENCE OF THE ANISOTROPY CONSTANTS 
magnetization and the c-axis, and the higher order terms are neglected. The energy is isotropic in the basal plane perpendicular to the c-axis. Results of some of their measurements are presented in Fig. 14。 It is believed that the primary sources of this anisotropy are dipole interaction and crystal field splitting. The region where the anisotropy changes sign is of interest to us. Here $K_{2}$ has a small positive value and $K_{1}$ appears to have a Iinear temperature dependence. For pure $\mathrm{Mn}_{2} \mathrm{Sb}$ the compensation point $-\mathrm{K}_{1}=\mathrm{K}_{2}$ occurs at $24 \mathrm{\gamma}^{\circ} \mathrm{K}$ 。 In the region between $-\mathrm{K}_{1}=2 \mathrm{~K}_{2}$ at $244^{\circ} \mathrm{K}$ and $\mathrm{K}_{1}=0$ at $250^{\circ} \mathrm{K}$, the easy axis of magnetization rotates continuously from the c-axis at high temperatures into the basal. plane at low temperatures. The intensity of diffraction peaks in the neutron diffraction measurements by Wilkinson et al. 7 changed continuously in this temperature range. It was assumed that this verified the existence of a continuous rotation of the axis of magnetization: The spin echo measurements which we w1ll present suggest that the behavior in the reorientation region is more complex.

\section{Theory}

Horner and Varma ${ }^{5}$ consider the expansion of the free energy 


$$
\begin{aligned}
F(T, H) & =F_{0}(T, H)+E_{A} \\
& =F_{0}(T, H)+K_{1} \sin ^{2} \theta+K_{2} \sin ^{4} \theta
\end{aligned}
$$

in a Landau-type description where $\theta$ is treated as an order parameter and $F_{0}, K_{1}$, and, $K_{2}$ are assumed to be analytic functions. The value of $\theta$ for different temperatures is determined by minimizing the free energy with respect to $\theta$. Therefore we solve

$$
\frac{\partial F}{\partial \theta}=2 K_{1} \sin \theta \cos \theta+4 K_{2} \sin ^{3} \theta \cos \theta=0
$$

and

$$
\frac{\partial^{2} F}{\partial \theta^{2}}>0
$$

The solutions to (1) are $\theta=\pi / 2, \theta=0$, and $\theta$ given by $\sin ^{2} \theta=-K_{1} / 2 K_{2}$. This last solution is real only for $0 \leqslant-\mathrm{K}_{1} / 2 \mathrm{~K}_{2} \leqslant 1$, 1. e., within the reorientation region. Assuming that $K_{1}$ is linear in this region we can write

$$
\mathrm{K}_{1}= \pm 2 \mathrm{~K}_{2} \frac{\left(\mathrm{T}-\mathrm{T}_{2}\right)}{\left(\mathrm{T}_{2}-\mathrm{T}_{1}\right)} \quad, \quad \mathrm{K}_{2} \geqslant 0,
$$

where the temperatures $T_{1}$ and $T_{2}$ are defined by $K_{1}\left(T_{2}\right)=0$ and $\mathrm{K}_{1}\left(\mathrm{~T}_{1}\right)=-2 \mathrm{~K}_{2}$, for $\mathrm{K}_{2}>0$. Then the solutions for the free energy are

$$
\begin{array}{ll}
F=F_{0}(T, H) & (\text { for } \theta=0), \\
F=F_{0}(T, H)+K_{1}+K_{2} & \text { (for } \theta=\pi / 2),
\end{array}
$$

and

$$
F=F_{0}(T, H)-K_{1}^{2} / 4 K_{2}
$$




$$
=F_{0}(T, H)-K_{2}\left[\frac{\left(T-T_{2}\right)}{\left(T_{2}-T_{1}\right)}\right]^{2} \quad \text { (for } \sin ^{2} \theta=\frac{\bar{K}}{\left.-K_{1} / 2 K_{2}\right)} \text {. }
$$

We have sketched curves for $F-F_{0}(T, H)$ in Fig, 15. For $\mathrm{K}_{2}<0$, the reorientation occurs in the interval between $\mathrm{T}_{2}$ and $\mathrm{T}_{1}^{i}$, where $\mathrm{K}_{1}\left(\mathrm{~T}_{1}^{i}\right)=-2 \mathrm{~K}_{2}$. The solid lines are drawn to show the minimum value of F-Fo $(T, H)$ which is the stable solution of the system. For $\mathbb{K}_{2}<0$, the system undergoes a first-order phase transition at $T=\frac{1}{2}\left(T_{1}+T_{2}\right)$ where $\theta$ shifts discontinuously from $\pi / 2$ to 0 .

In our samples of $\mathrm{Mn}_{2-\mathrm{x}} \mathrm{Cr}_{\mathrm{X}} \mathrm{Sb}$ we have $\mathrm{K}_{2}>0$. In the reorientation region the angle $\theta$ satisfies the equation

$$
\sin ^{2} \theta=-\mathrm{K}_{1} / 2 \mathrm{k}_{2}
$$

and the free energy has continuous first derivatives。. In Fig. 16(b) we show $\theta$ as a function of temperature. The slope of the curve is given by

$$
\frac{d \theta}{d T}=\left[2\left(T_{2}-T_{1}\right) \sin \theta \cos \theta\right]^{-1}
$$

which becomes infinite at $\theta=\pi / 2$, 0 . In the Landau description a discontinuous change in the first derivative of the order parameter, $\theta$, is associated with a second-order phase transition. With the se transitions we expect a discontinulty in the specific heat at $T_{2}$ and $T_{1}$ given by

$$
|\Delta C(T)|=T \frac{\partial^{2} F}{\partial T^{2}}=T\left[\frac{2 K_{2}}{\left(T_{2}-T_{1}\right)}\right] \text {. }
$$




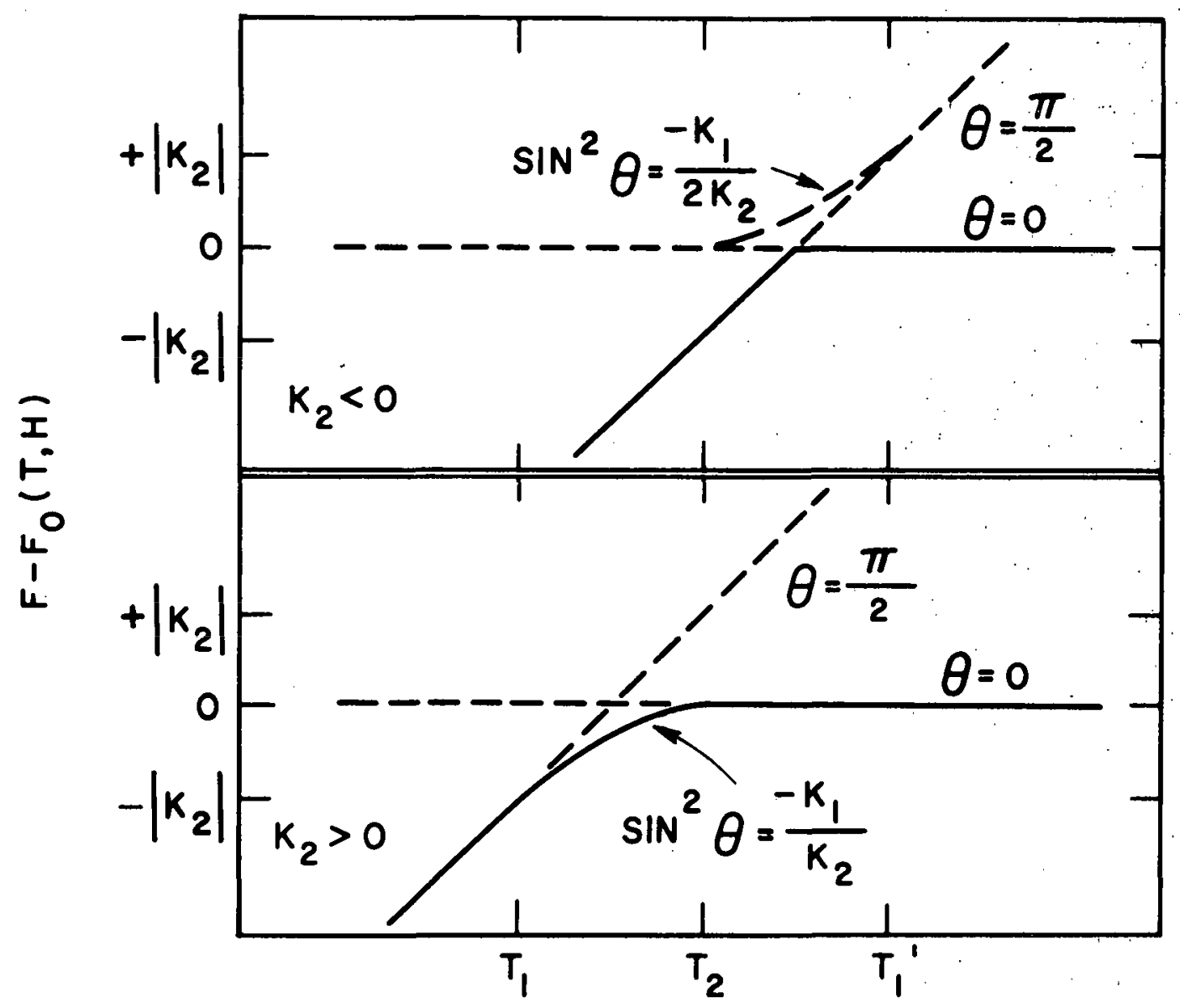

FIGURE 15. ANISOTROPY FREE ENERGY IN THE REORIENTATION REGION 


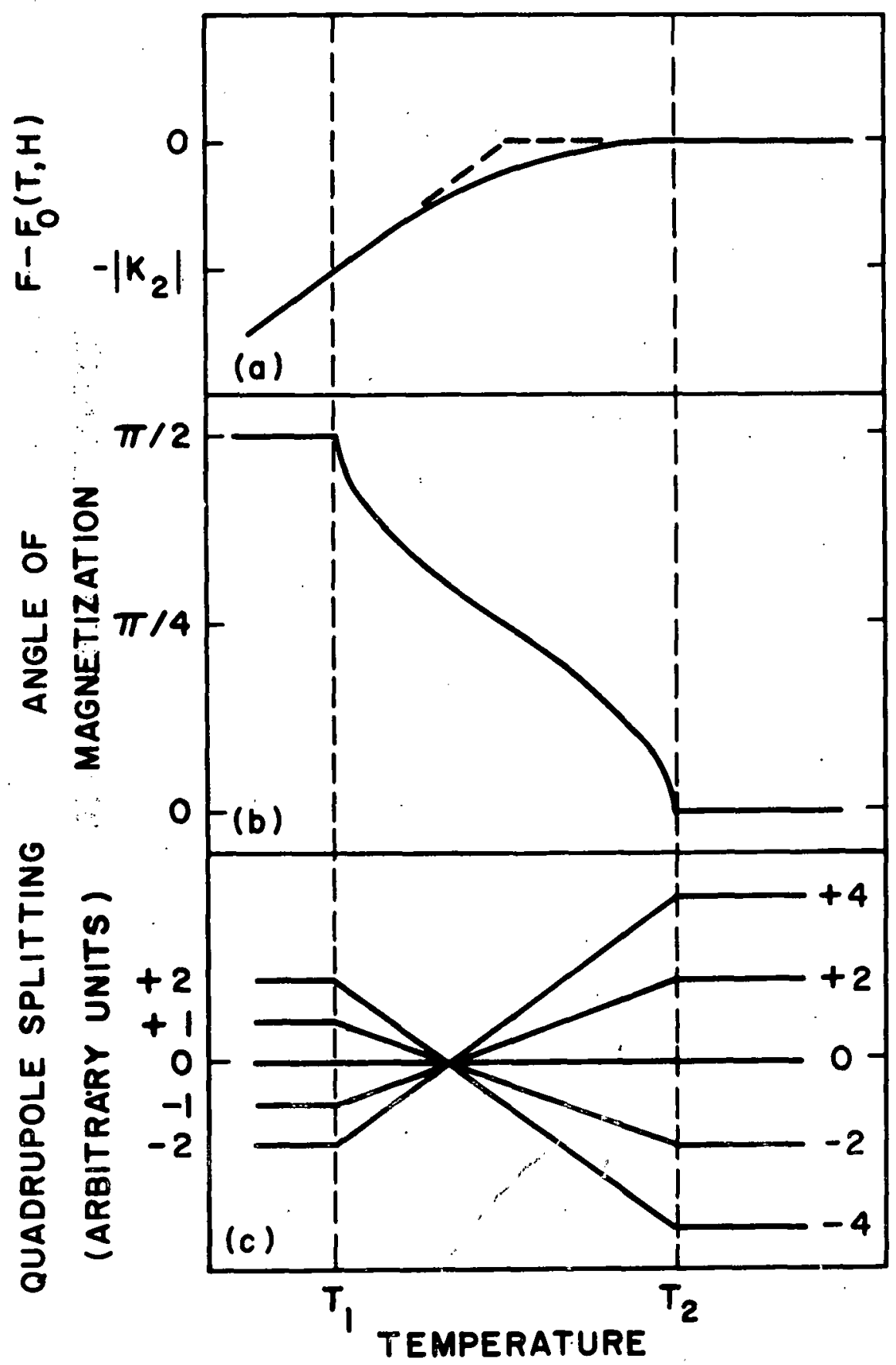

FIGURE 16. PREDICTED BEHAVIOR OF THE MAGNETIZATION OR IENTATION AND Q QUADRUPOLE SPLITTING IN THE REORIENTATION REGION FOR $\mathrm{K}_{2}>0$ 
For $\mathrm{Mn}_{2} \mathrm{Sb}$ where $\mathrm{T}_{2}-\mathrm{T}_{1}=6^{\circ} \mathrm{K}, \quad \mathrm{T} \approx 247^{\circ} \mathrm{K}$, and $\mathrm{K}_{2} \approx 3 \times 10^{4}$ ergs $/ \mathrm{cm}^{2}$, we get $\Delta \mathrm{C} \approx 0.006 \mathrm{~J} / \mathrm{g}^{\circ} \mathrm{K}$ or about $2 \%$ of the lattice specific heat.

Recent studies on rare earth orthoferrites at. their spin reorientation temperature by $R$ 。 $C$. LeCraw et al. 4 revealed a strange broadband ( $5 \mathrm{MHz}$ to $54 \mathrm{GHz}$ ) microwave absorption. This behavior suggests the presence of some sort of fluctuation in the spin system which is consistent with a second-order phase transition. $J: R$. Shane ${ }^{64}$ has analysed the spin reorientation in orthoferrites and obtains the same form for the minimum free energy as Horner and Varma. He proposed that the broadband absorption arises from a smearing out of strongly temperature dependent spin wave resonance freqúencies.

The prediction by Horner and Varma of the two second-order phase transitions depends on the fact that $\mathbb{K}_{1}$ varies linearly with $T$ or is at least analytic at $\mathrm{T}_{1}$ and $\mathrm{T}_{2}$ 。 Although the duPont anisotropy data suggests linear behavior, no anisotropy measurements were made between $T_{1}$ and $T_{2}$. However, the fact that the HornerVarma theory is also able to describe the magnetic field dependence of the absorption measurements of Lecraw et al. Strengthens their assumptions of the behavior of $\mathrm{K}_{1}$ in the reorientation region.

From the spin echo modulation we are able to 
measure the quadrupole splitting. Its behavior in the spin reorientation region is easily derived as follows. The quadrupole splitting is proportional to the crystal field $V_{z Z}$. In a tetragonal crystal

$$
V_{z z} \propto \cos ^{2} \theta-\frac{1}{2} \sin ^{2} \theta=1-\frac{3}{2} \sin ^{2} \theta \text {. }
$$

Since $\sin ^{2} \theta=\frac{\left(T_{2}-T\right)}{\left(T_{2}-T_{1}\right)}$, we have

$$
V_{z z} \infty 1-\frac{3\left(T_{2}-T\right)}{2\left(T_{2}-T_{1}\right)}
$$

The quadrupole splitting shown in Fig. 16(c) wili double in size as the easy axis rotates from the basal plane to the c-axis.

\section{Experimental Measurements}

Information on the behavior of the $\mathrm{Mn}_{2-x} \mathrm{Cr} \times \mathrm{Sb}$ near the reorientation region is obtained from measurements of the modulation of the envelope of the spin echo maxima. From our discussion in Chapter II we recall that the decay of the echo is modulated by the function

$$
a+b \exp \left[-2 T / T^{*}\right] \cos \left[\left(2 \pi / \tau_{m}\right)+\delta\right] \text {. }
$$

We are interested in measurements of the modulation period $\tau_{m}$ and the percent of amplitude modulation $100\left[\mathrm{~b} \exp \left(-2 \tau / \mathrm{T}^{*}\right) / \mathrm{c}\right]$.

The factor $\exp \left(-2 \tau / \mathbb{T}^{*}\right)$ is a damping term that was 
omit.ted in the formal calculations of spin echo modulations that we have reviewedo. The decay time $I^{*}$ is

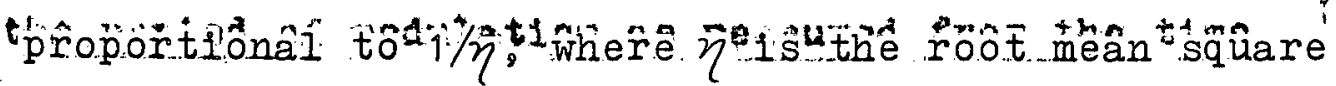

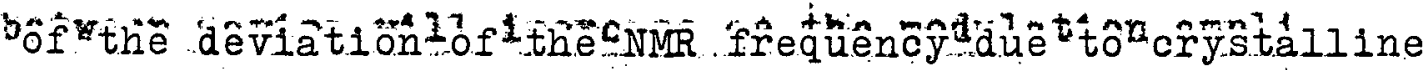

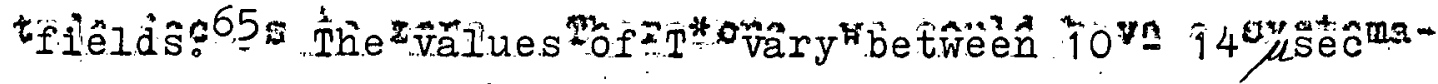

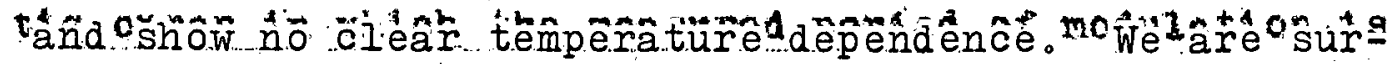

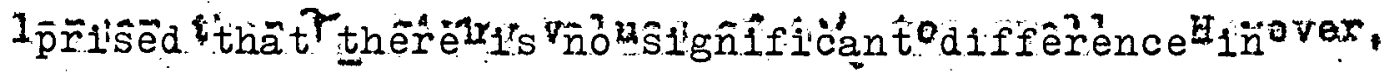

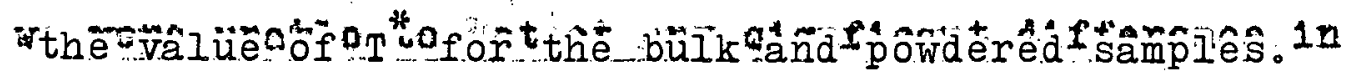

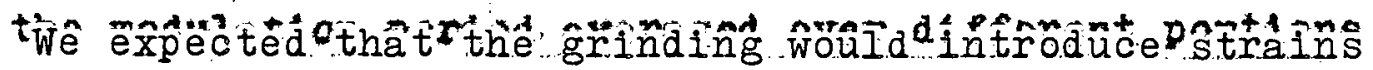

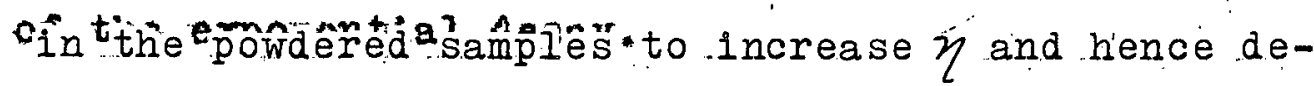
crease To oertaln that those measurenenta are villa,

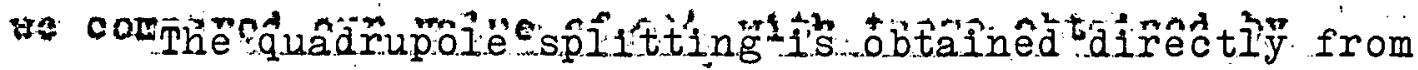

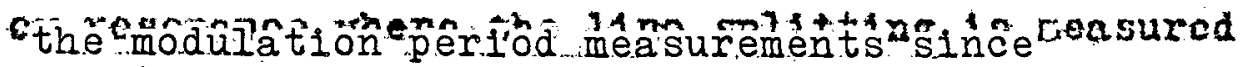
directis: Isrginel oecliletor poasuromonts of the

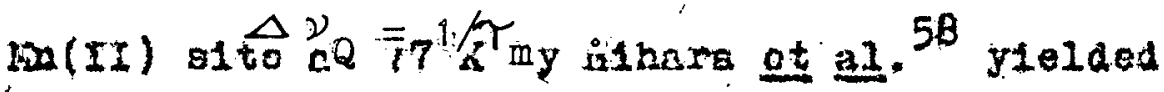

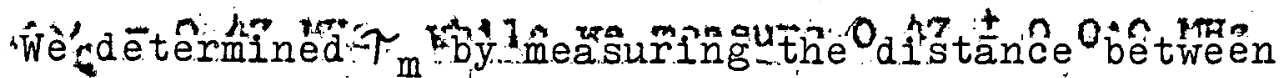

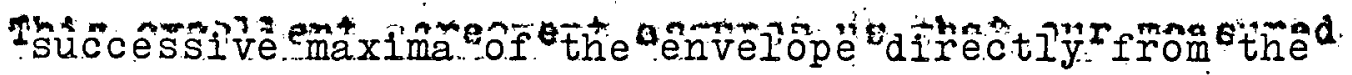

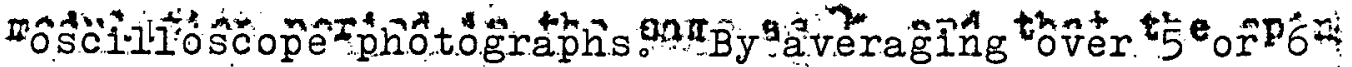

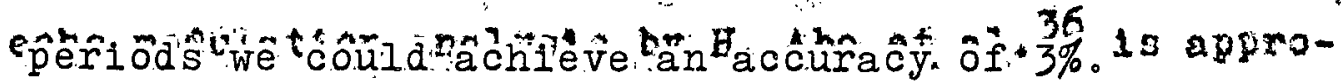

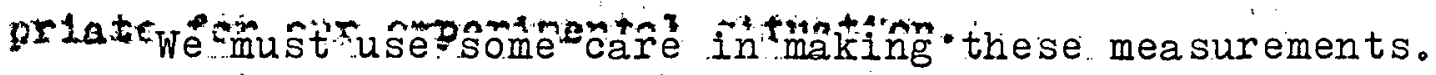

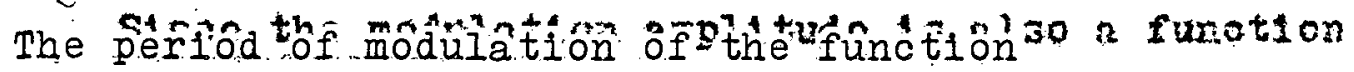
of $\operatorname{cop}\left(-2 T / 2^{*}\right)$, we made all meagurements of the parout of madulacos $\left[\left(2 \pi / \tau_{1}\right) \tau\right] \exp \left(-2 \tau / T_{2}\right) \%$ Intorpretation

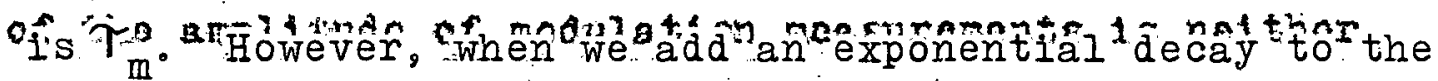

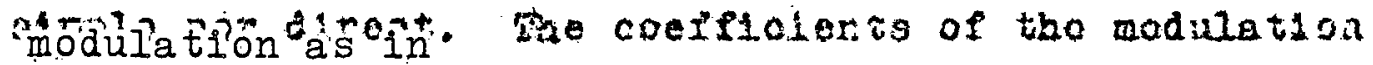
teras caloulatod by H. Ake et al. are the absoluto 


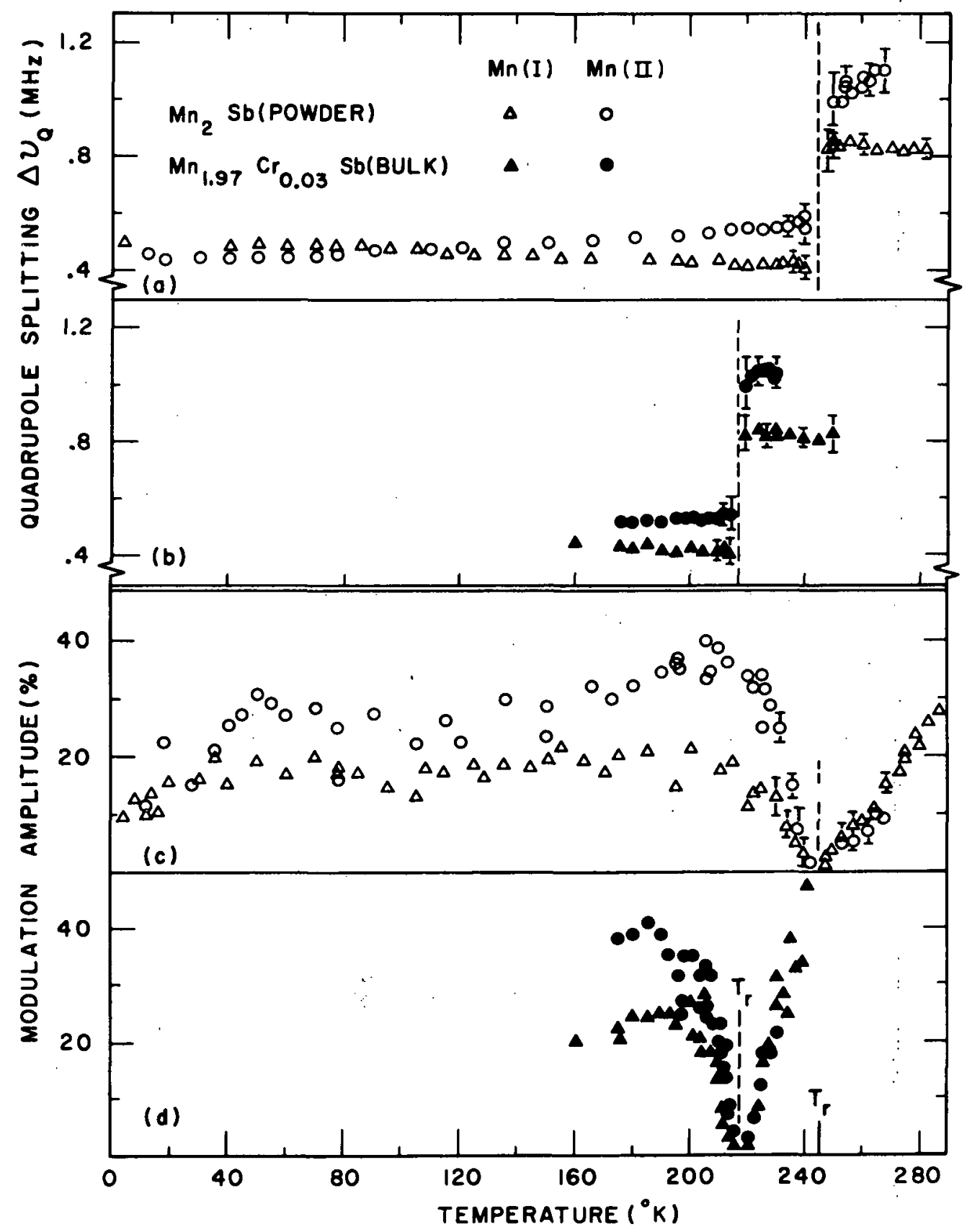

FIGURE 17. TENRERATURE DEPENDENCE OF THE JUADRUPOLE JPLITTING AUD THE JPIN EOHO MODULATION A:TPITUDE 


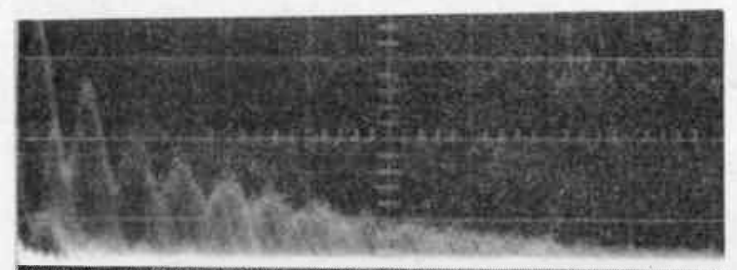

TEMPERATURE ${ }^{\circ} \mathrm{K}$

230
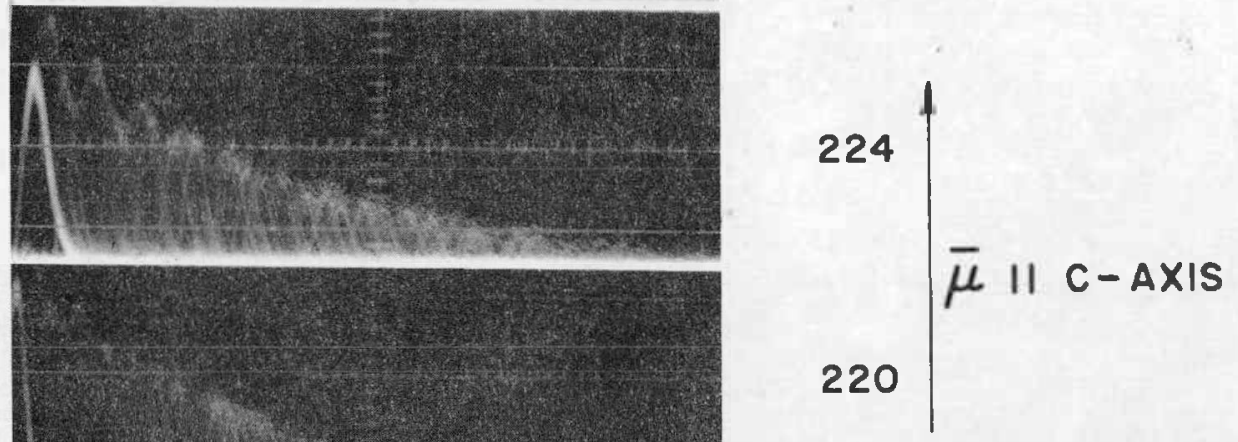

216
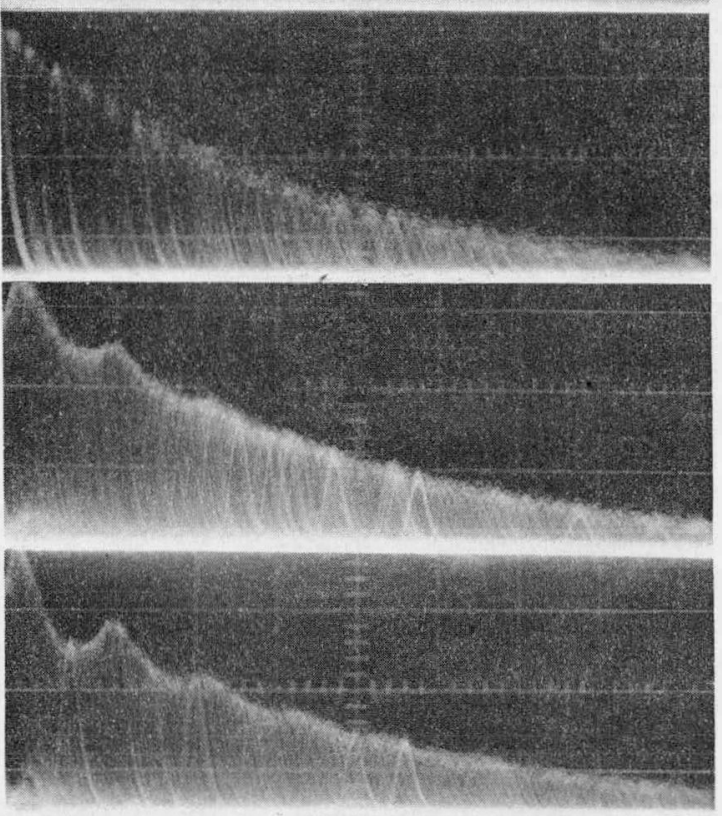

$\bar{\mu} \perp \mathrm{C}-\mathrm{AX} \mid \mathrm{S}$
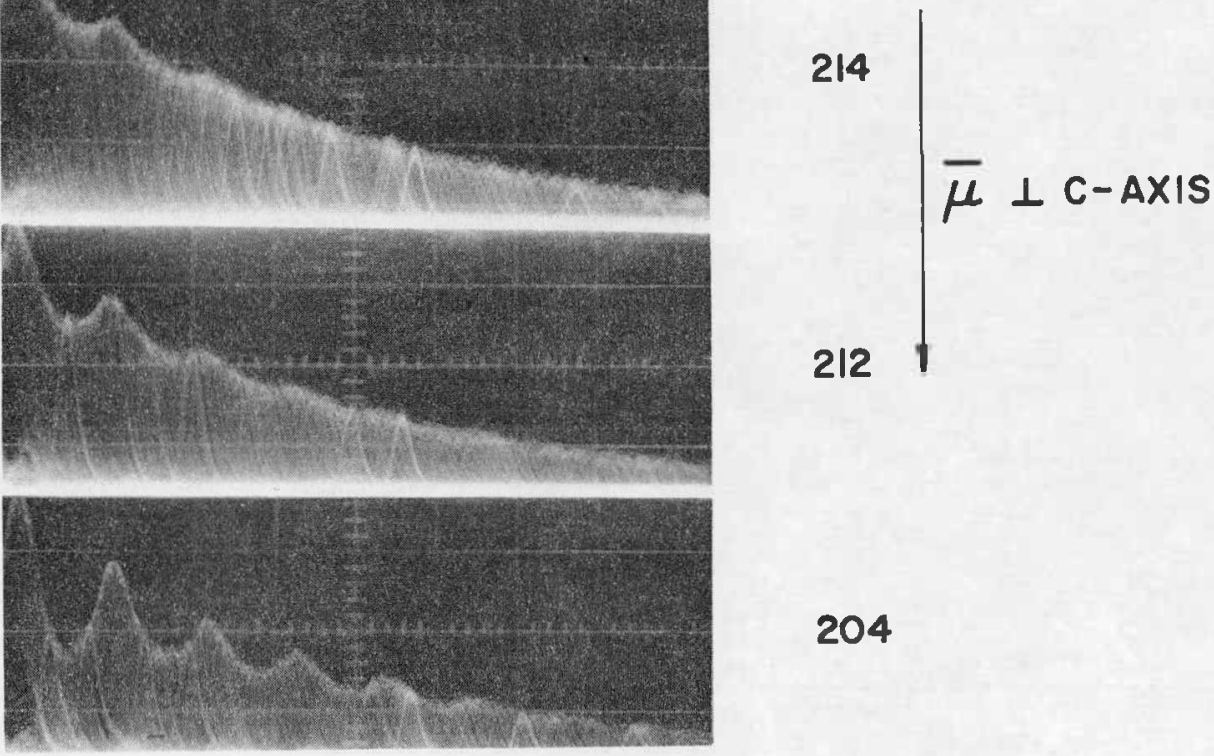

204

HORIZONTAL TIME SCALE $2 \mu$ SEC/DIV

FIGURE 18. PHOTOGRAPHS OF TIIE SPIN ECHO ENVILOPE INEAR Tr OF TIE $\operatorname{lin}(\mathrm{I})$ SITE IN $\mathrm{Mn}_{1.97^{\mathrm{Cr}} \mathrm{O} .03^{\mathrm{Sb}}}$ 
where the modulation amplitude becomes very. small. This limits the number of periods that can be observed and makes the peaks of the modulation less distinct. At high temperatures the error again increases because the amplitude of the spin echo is rapidly decreasing. In the region $\mathrm{T}_{\mathrm{r}} \pm 3^{\circ} \mathrm{K}$ we did not attempt to make any measurements of $\Delta \nu_{Q}$. Near this region there is no indication of a gradual change in the modulation period or a mixing of two modulation frequencies. The change in the quadrupole splitting,

$$
\Delta \nu_{Q}\left(T>T_{r}\right)=2 \Delta \nu_{Q}\left(T<T_{r}\right),
$$

is exactiy what we expect for a quadrupole interaction in a uniaxial crystal.

The behavior of the two samples is remarkably similar considering the differences in their preparation. The narrower transition region for $\mathrm{Mn}_{1.97^{\mathrm{Cr}}} 0.03^{\mathrm{Sb}}$ in Fig.17(d)is due to the fact that this is a bulk sample with greater homogeneity and fewer strains. The shift in $T_{r}$ from $x=0$ to $x=0.03$ is exactly what we expect from the anisotropy measurements in Fig. 14. With the bulk sample, we applied an external magnetic field up to 1 koe perpendicular to the c-axis. At temperatures just above $\mathbb{T}_{r}$ we were able to pull the magnetic moments back into the c-axis and shift $\mathrm{T}_{\mathrm{r}}$ to $a$. higher temperature. 
An interesting feature in Fig. $17(c)$ and (d) above $T_{r}$ is that the magnitude of the modulation amplitude of the $M n(I)$ site increases to values above the maximum value measured below $T_{r}$. Since the quadrupole splitting a above $T_{r}$ is twice that below. $T_{r}$, it indicates that the modulation amplitude is a function of a as we expect. Unfortunately the rapid decrease of the echo amplitude prevented measurements at higher temperatures to see at what ivalue the modulation amplitude levels off. Interpretation of the data in Fig. 17 presents us with a dilemma. How do we account for the large change in modulation amplitude over the region $\Delta \nu$ remains constant? As the axis of magnetization rotates between $\theta=\pi / 2$ and $\theta^{\prime}=0$, the quadrupole splitting should pass through a zero and we expect to see $\Delta \nu_{Q} \rightarrow 0$ in this region. There is no indication of any deviation of $\Delta \nu_{Q}$ to within $3^{\circ}$ of $T_{r}$. In Fig. 17(d) we se'e that at $\mathrm{T}_{\mathrm{r}}-7^{\circ} \mathrm{K}$ the modulation amplitude is half of its maximum value: Inhomogeneity of the or doping in the bulk sample can account for a broadening of the transition temperature by a few degrees but not to the width which we observe which approaches $20^{\circ} \mathrm{K}$.

It is difficult to give an exact and detailed explanation of these results. It seems reasonable to suggest that a spatial instability in the orientation of the magnetization reduces the modulation amplitude 
over a wide temperature range. The modulation is almost completely washed out before there is any. indication of a change in the orientation. Our data does not give us any information on the nature of the reorientation at $\mathrm{T}_{\mathrm{r}^{\circ}}$

We made a careful study of $T_{2}$ in this region to see if changes in the line width could account for the change in modulation amplitude. The data are shown in Fig. 19. The Mn(I) site with a smaller $T_{2}$ has the larger modulation amplitude which suggests that the line width is a factor. However, there are no changes in $T_{2}$ of sufficient size to explain the dramatic change in modulation near $T_{r}$. There are small peaks in $T_{2}$ that are statistically significant at $T_{r}$ for $M(I)$ and above $T_{r}$ for $\operatorname{Mn}$ (II). We are unable to explain their presence. They may be associated with the fluctuations in the spin system.

Finally in Fig. 20 we show a detail of $\mathcal{V}(I)$ and $\nu$ (II) in the reorientation region. The lines drawn in the figure are least square fits to the data above and below $T_{r}$ to show more clearly the displacement in the NMR frequency. Since magnetization measurements on the bulk sample with c-axis perpendicular to an applied field $H_{0}$ show no spurious behavior at $\mathrm{T}_{r}$ even for $\mathrm{H}_{\mathrm{O}} \leqslant 1 \mathrm{kOe}$, the displacement in $\nu$ must be caused by an anisotropy in the hyperfine interaction. The 


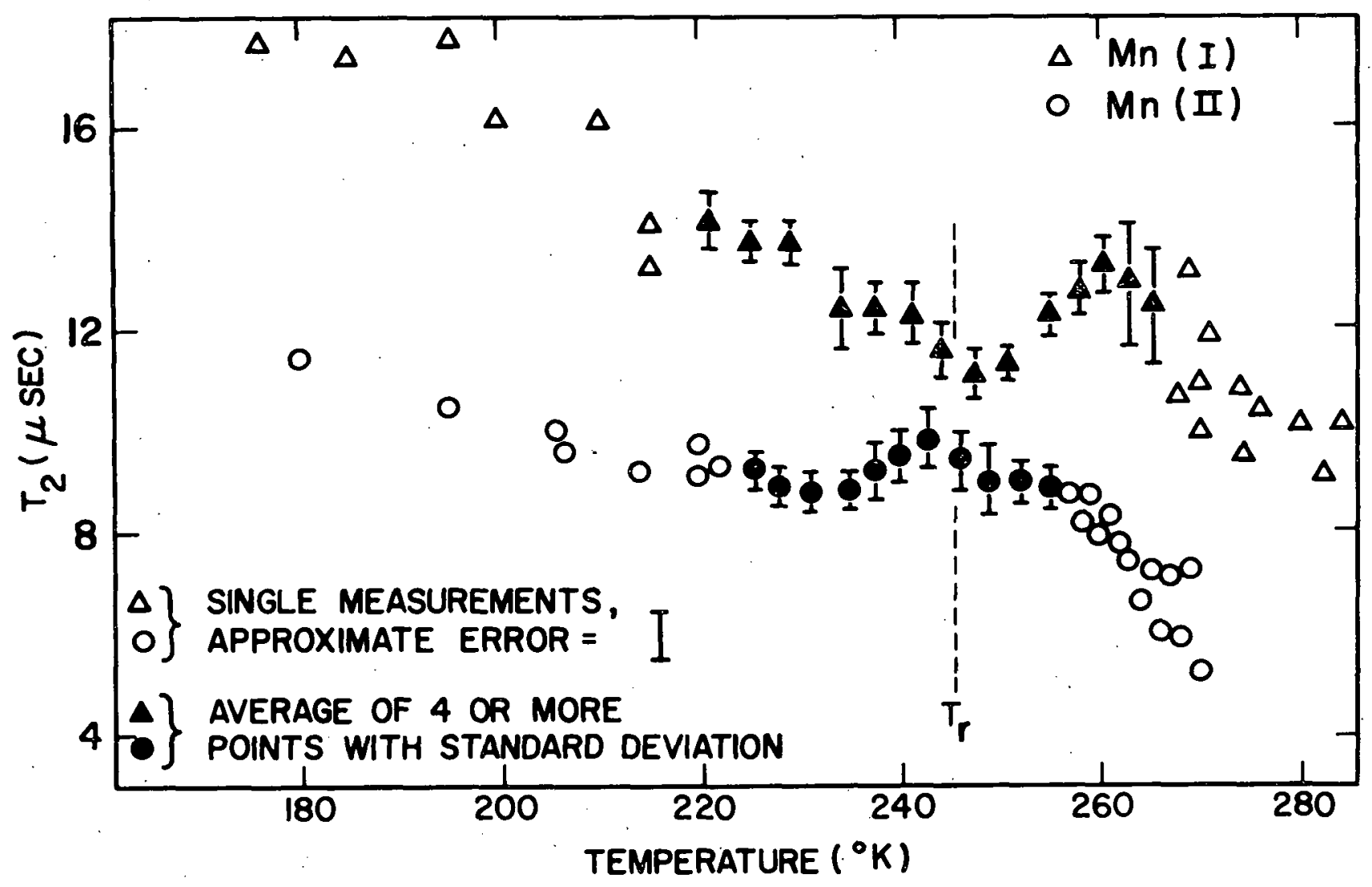

FIGURE 19. TEMPERATURE DEP INDENCE OF $\mathrm{T}_{2}$ NEAR $\mathrm{T}_{\mathrm{r}}$ 


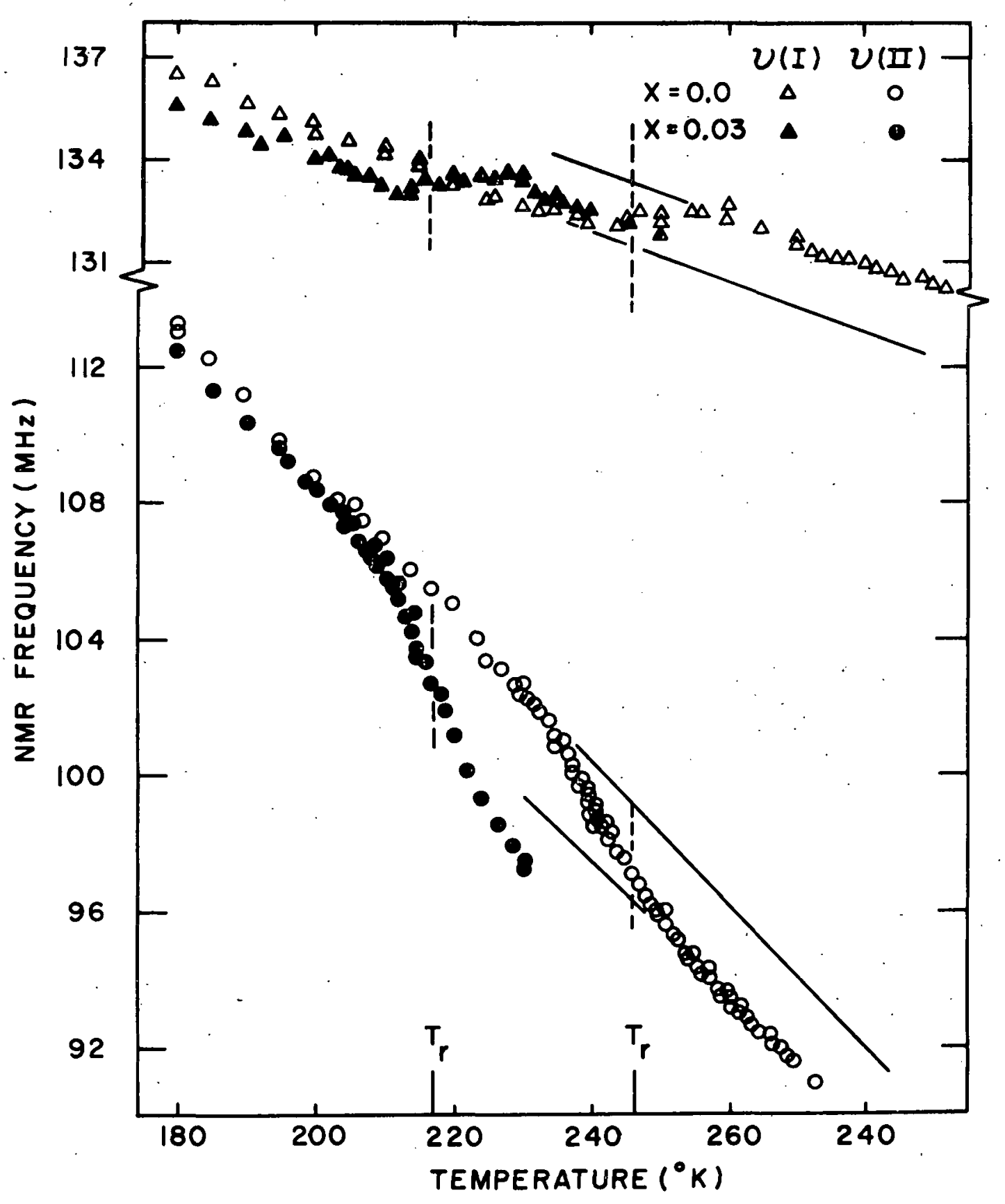

FIGURE 20. TEMPERATURE DEP ENDENCE OF THE NMR FREQUENCY NEAR $T_{r}$ 
displacements of $\nu(I)$ and $\nu$ (II) are in the opposite direction as expected for a ferrimagnetic structure.

It is difficult to reconcile these NMR frequency measurements with the quadrupole splitting measurements. These should be a measure of the dominant value of the magnetic and electric fields respectively at the nuclear sites. The displacement in the NMR frequency takes place over a $20^{\circ} \mathrm{K}$ interval. While the quadrupole splitting shifts in a region less than $6^{\circ} \mathrm{K}$ wide. We are unable to explain the reason for this difference.

\section{Relationship of Experimental Results to the Horner-}

\section{Varma Theory}

The work of Horner and Varma ${ }^{5}$ demonstrates that from thermodynamic principles a continuous change in $\theta$ must take place. In $\mathrm{Mn}_{2} \mathrm{Sb}$ the rotation takes place over a $6^{\circ} \mathrm{K}$ interval. Unfortunately this is exactly the region in which we were unable to make reliable measurements. Even if we were able to measure into the reorientation region, a broadening of $\mathrm{T}_{\mathrm{r}}$ by $1^{\circ}$ or $2^{\circ} \mathrm{K}$ due to inhomogeneities in the sample would prevent us from seeing any: continuous change in $\Delta \nu_{Q}$. The two secondorder phase transitions that have been predicted might account for the behavior of the modulation amplitude. Associated with these transitions is: a fluctuation "of the order parameter. A theory which develops a dynamic 
model of the reorientation is needed to describe this behavior in detall.

It is clear that a metal is not a good material for a study of the spin reorientation. With an insulator, NMR measurements could be made on large single crystals to assure homogeneity and exact lattice orientation. The rare-earth orthoferrites, in which the spin reorientation has already been observed, would be sultable. The major problem is in obtaining a suitable nuclear site to observe a IMR signal. ...There is also a problem in growing large crystals without the crystal imperfections known as twinning, where $90^{\circ}$ domain walls are formed. There is already a sufficient amount of interesting experimental phenomena and theoretical speculation on the spin reorientation region to warrant further study.

In an attempt to verify the existence of the two second-order phase transitions, we looked for the specific heat anomally predicted by Horner and Varma. A brief description of the apparatus is given in Appendix $D$. We were able to obtain data with a scatter of $1 \%$ but observed no anomalous change in the specific heat. As is clearly pointed out by Horner and Varma in their paper, if the assumption of the linearity of $\mathrm{K}_{1}$ breaks down at $T_{1}$ and $T_{2}$,more complicated behavior is expected and the predicted specifc heat anomalies may be altered. 
It seems that this research has raised more questions than it has answered. There are several reasons for our inability to draw specific conclusions from our data. First, $\mathrm{Mn}_{2-\mathrm{x}} \mathrm{Cr}$ Sb has a complicated magnetic structure and a mixture of ionic and covalent bonding. Secondly, the theory of ferromagnetic metals is not sufficiently developed to provide a detailed interpretation of our relaxation time data.

The rf power and magnetic field dependence of the nuclear spin echoes is consistent with other research on ferromagnetic metals. We are able to distinguish the echoes from the nuclei in the domain and the domain walls. The nuclear relaxation times are very short, but are nearly the same magnitude as those measured in Co. Much more data. will be needed on other ferromagnetic materials to understand the mechanism for the fast nuclear relaxation which has been observed.

our most confusing data are the NMR frequency measurements. They do not even agree qualitatively with the predictions of spin wave theory or molecular field sublattice magnetization calculations. The strange Iinear temperature dependence of $\nu$ (I) may be due to a strong temperature dependence of the electronic states. 
The most significant result of this research is the study of the spin reorientation at $T_{r}$. We found an indication of a fluctuation in the spin system over a wide interval about $\mathbb{T}_{\boldsymbol{r}}$. This is consistent with the prediction of two second-order phase transitions. To understand the nature of these fluctuations, a dynamic model of the spin reorientation is required. Because of the inhomogeneous distribution of crystalline flelds due to crystal strains and a broadening of $T_{r}$ due to an inhomogeneity in the or concentration in our sample, we are not able to study the behavior of the reorientation at $T_{r}$. To make a critical test of the nature of the spin reorientation, it is important that this region be studied in a very homogeneous material. Since spin reorientation occurs in many materials, this work should have wide applicability. 
Foner Magnetometer Design

The schematic diagram on page A-5 shows the important features of the magnetometer construction. The vibrating rod is $3 / 16 " 0 . d_{0}, 0.010^{\prime \prime}$ wall stainless steel tube centered in a stainless steel vacuum jacket by teflon rings. These rings are irregularly spaced to suppress any transverse waves in the rod. The reference co1l is made from 200 turns of \#30 Evanohm wire wound on a spool 0.15" in diamater and 0.435" long. The spool is mounted securely in a rectangular yoke on the top of the rod: The heater consists of \#30 constantan wire wound directly on the copper piece containing the sample at the bottom of the rod. The copper-constantan thermocouple junction is embedded in a hole in the copper using epoxy with alumina powder filler for good thermal contact. A $5 \mathrm{kHz}$ oscillator provides a maximum power of one watt to the heater.

The pickup colls have the dimensions: 0.2" 1.d., $0.4 "$ " o.d., and 0.5" long. Each coil has 3400 turns of \#40 copper wire and a resistance of 300 at room temperature. Pairs of coils are mounted around the sample and reference coil as shown in the cross section drawing on page A-5. The two coils are wound in opposite directions so that the signals finduced by the vibrating dipole in each coil are added while signals from other sources are subtracted. Radiation from the driver can be a major source of unwanted 
signal and usually requires careful shielding. The pickup coils are placed so that the vibrating dipole is in a saddle point such that the amplitude of the induced signal is insensitive to lateral displacements of the vibrating rod. The amplitude of the rod vibrations is about one millimeter.

The major source of spurious signal is from vibration of the pickup coils in the magnetic field. In order to minimize vibrations in the system the driver is mounted securely toa $2^{\prime \prime} \mathrm{x} 12^{\prime \prime} \mathrm{x} 12^{\prime \prime}$ aluminum block. The plckup colls are set in a brass block which is held tightly to the dewar wall by spring finger stock. This is carefully constructed so that the vacuum can does not touch the pickup coils. However, vibrations are coupled from the vacuum can to the coils through the Iiquid nitrogen in the dewar.

The detection system is shown in the block diagram on page A-6. Detall circuit diagrams are given of the constant current supply, the mixer, and the phase sensitive detector on page $A-7$ and $A-8$.

The current supply provides a wide range of constant current levels to the reference coll. The resistance values are chosen to provide maximum currents of 300 , $100,30,10,3$, and $1 . \mathrm{mA}$. Within each range the current level is controlled linearly by a precision 10 turn potentiometer. The milliammeter is included only to monitor the current. The setting of the potentiometer 
dial gives the current level with $0.1 \%$ accuracy. The phase sensitive bridge detector averages out any signal not at $150 \mathrm{~Hz}$. However, if these spurious signals are large, they will saturate the amplifier and reduce the sensitivity of the null detection. For this reason the detection circuit includes several filter stages. The $5 \mathrm{kHz}$ signal from the heater is sufficiently filtered so that it has no effect on the output of the bridge. The twin tee filter section is designed to filter out $60 \mathrm{~Hz}$ which is particularly troublesome。

The current in the microameter is proportional to the integral of the output signal of the phase sensitive bridge. The detector has an automatic gain control such that there is high sensitivity only near the null. The transistors across the UC=H6030 amplifier give an exponential feedback voltage. This provides logarithmic gain for input voltages between 0.1 and $100 \mathrm{mVpp}$ and protects the microammeter from overload. Unfortunately the gain of the amplifier is controlled by the instantaneous output level so that the gain is limited by the noise level. This reduces the sensitivity of the null detection. 


$$
A-4
$$

The microammeter has full scale readings of $\pm 25 \mu \mathrm{A}$. With $300 \mathrm{~mA}$ in the reference coil we produce a $2 \mathrm{mV} p \mathrm{p}$ signal which gives a meter reading of $18 \mu \mathrm{A}$. The null detector is insensitive to input voltages below $0.01 \mathrm{mV} \mathrm{pp}$ 。 


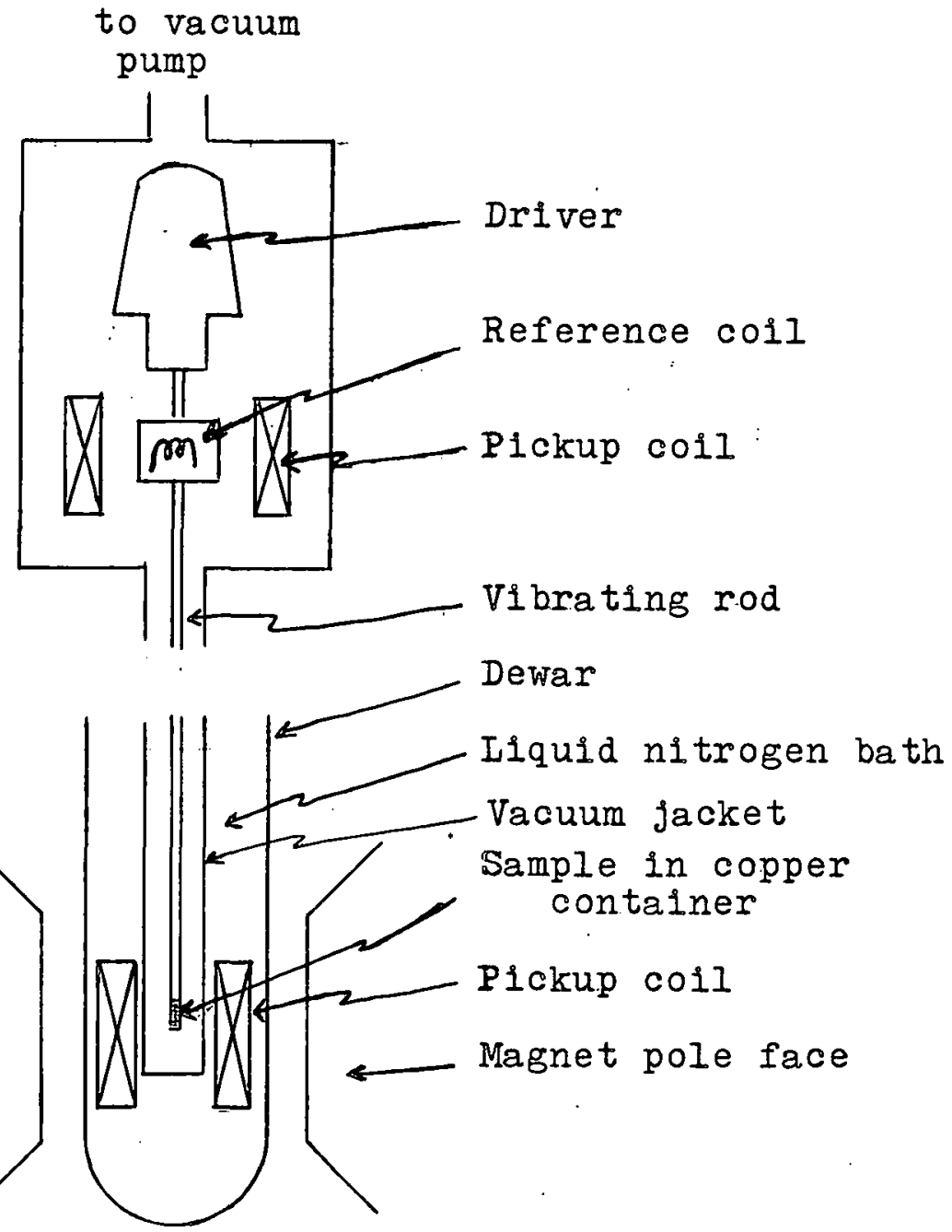

Schematic Longitudinal Section

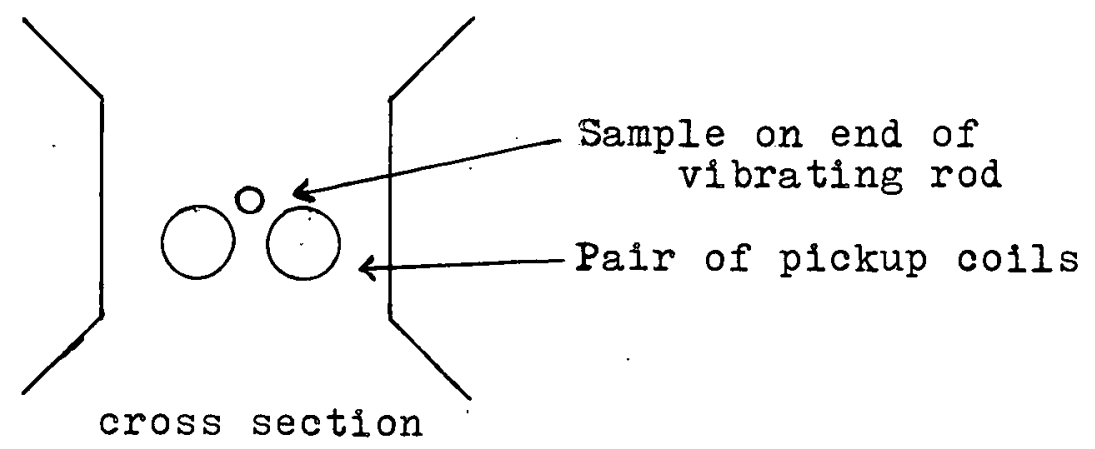

FONER MAGNETOMETER 


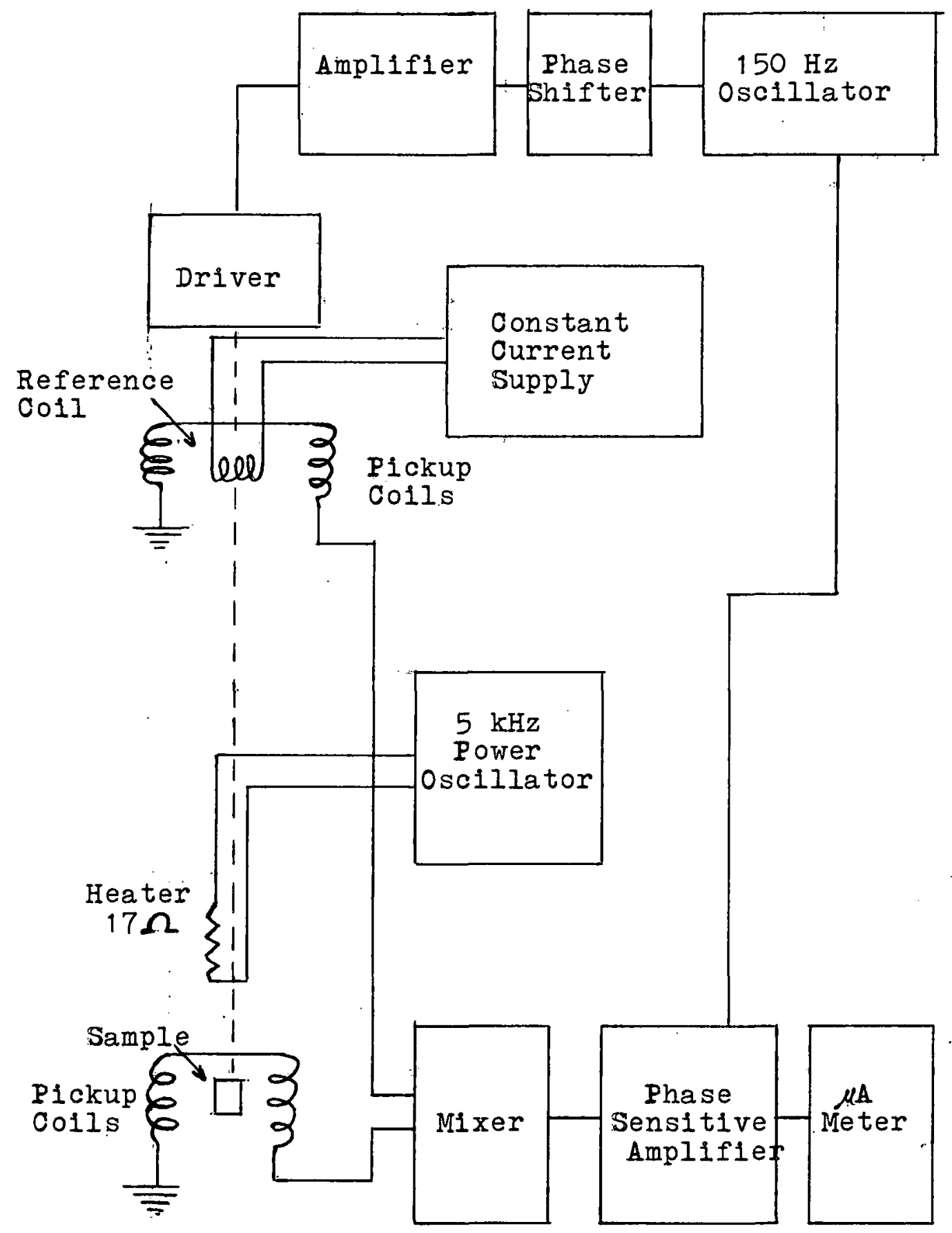

BLOOK DIAGRAM OF MAGNETOMETER 


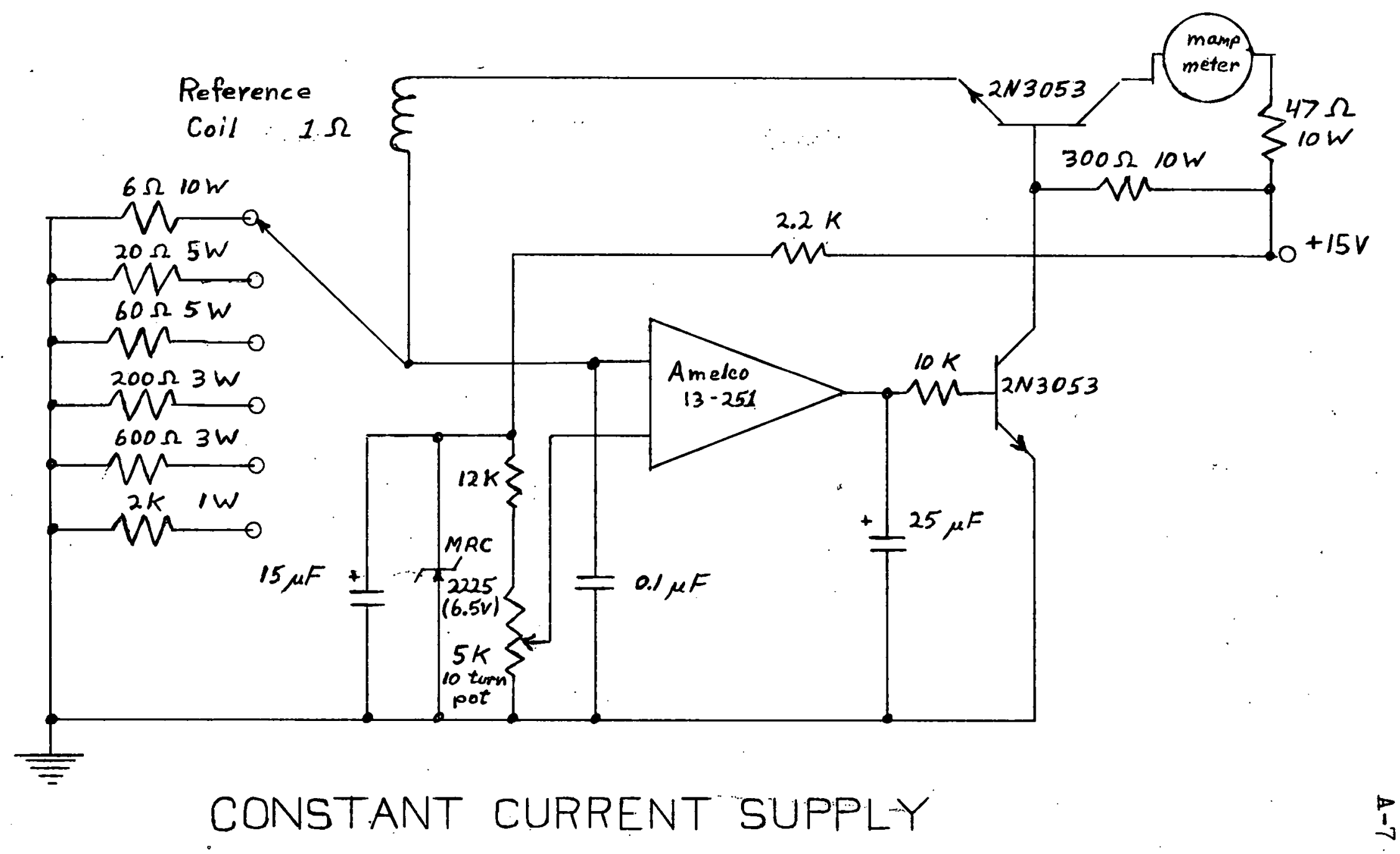




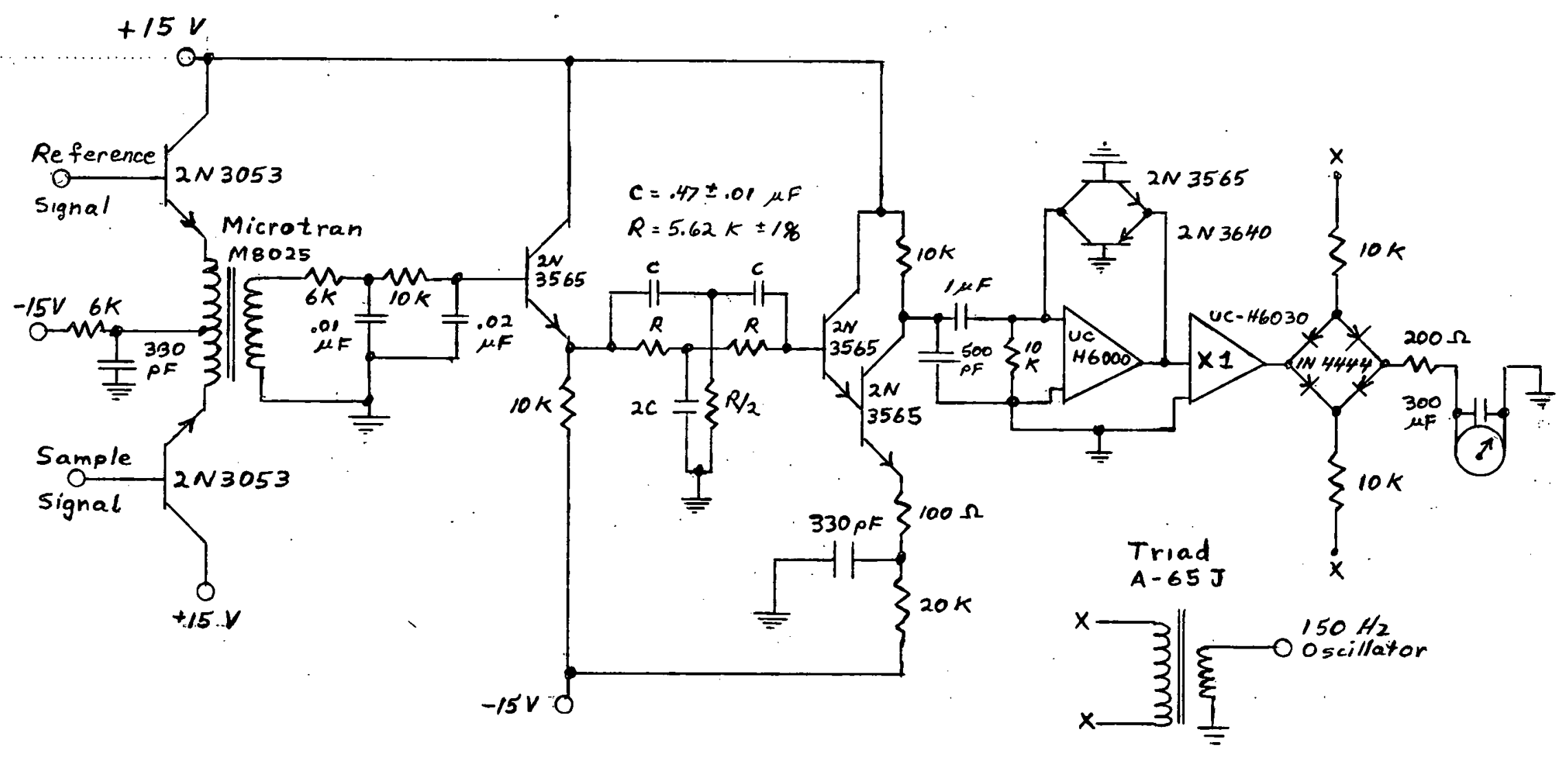

MIXER, AMPLIFIER, PHASE SENSITIVE BRIDGE 


\section{Oscilloscope Trace Blanking}

Blanking of the oscilloscope trace is achieved by applying $+50 \mathrm{~V}$ with respect to ground directly to the cathode of the cathode ray tube. The circuit shown on the next page is triggered by $-20 \mathrm{~V}, 50 \mathrm{nsec}$ sync pulses from the pullse generator. The $50 \mathrm{~V}$ blanking voltage is turned on when a sync pulse is applied to the ON input terminal. The blanking voltage is turned off after a delay interval following a sync pulse to the OFF input terminal. The delay interval can be varied between 1 and $4 \mu$ sec.

The primary difficulty with this blanking technique is that a bright spot appears on the oscilloscope trace as soon as the blanking voltage is removed. This is due to a build up of charge in the oscilloscope circuitry during the blanking interval. When long blanking intervals are required the intensity of the spot becomes too great to take clear photographs of the spin echo decay. For this reason we used the boxcar integrator to measure large values of $\mathbb{T}_{1}$. 


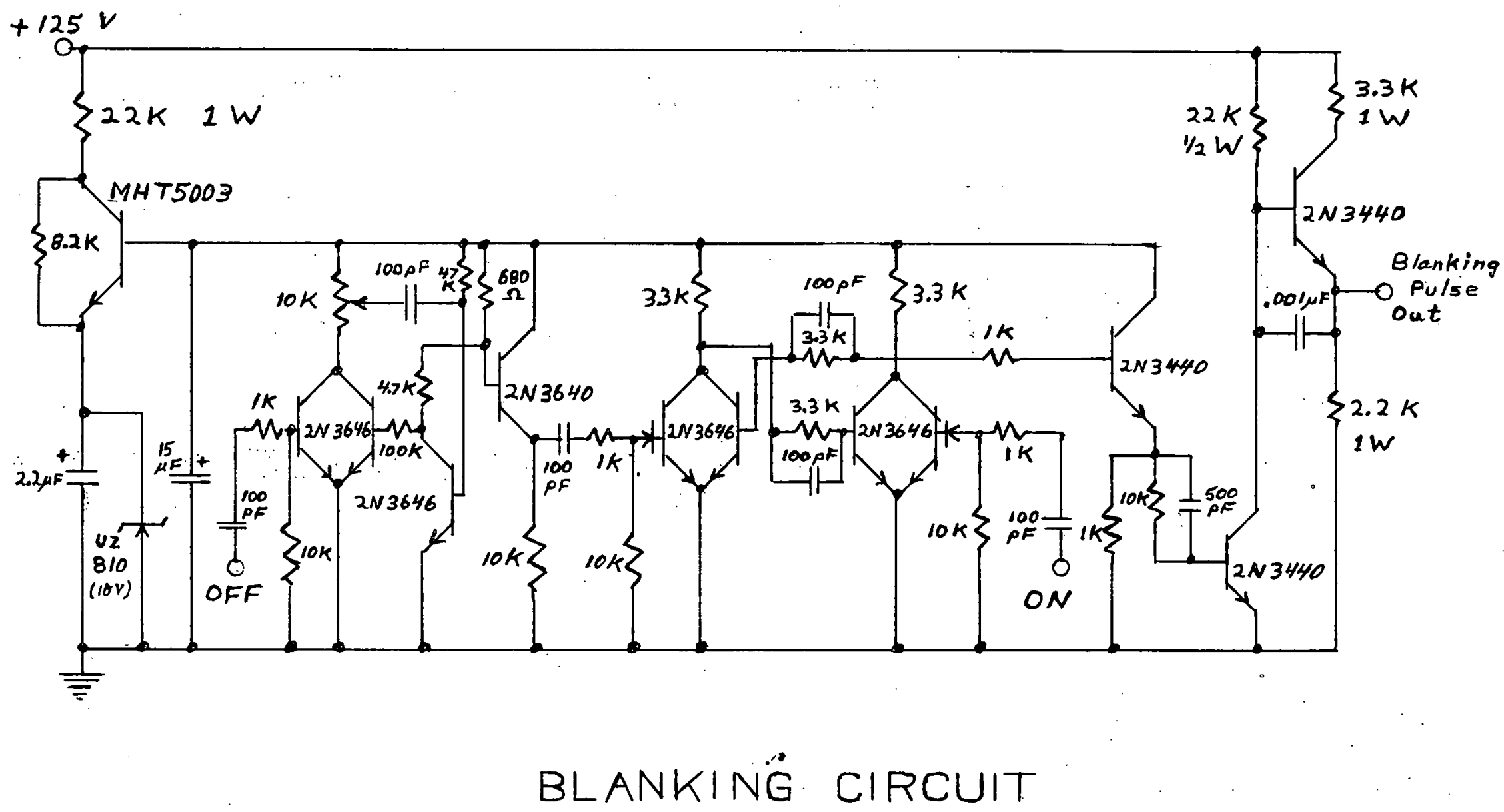


Magnetic Field Pulses

We show here the circuit used to produce magnetic field pulses. The gated diode is triggered by the output of the sync delay circuit (page $0-2$ ) which in turn is triggered by the pulse generator. The delay time can be varied between 1.7 and $26 \mu$ sec. This allows adjustment of the position the magnetic field pulse relative to the pulses of $r f$ power.

The circuit shown below is carefully constructed to allow rapid discharge of the capacitors, which is necessary to achieve a large field with a fast rise time. To minimize the resistance and inductance in the circuit, the diodes are bolted to brass blocks and all leads are made as short as possible with \#8 copper wire. Five metal film $1 \mu F$ capacitors are mounted in parallel. These can easily be removed when smaller pulses are desired.

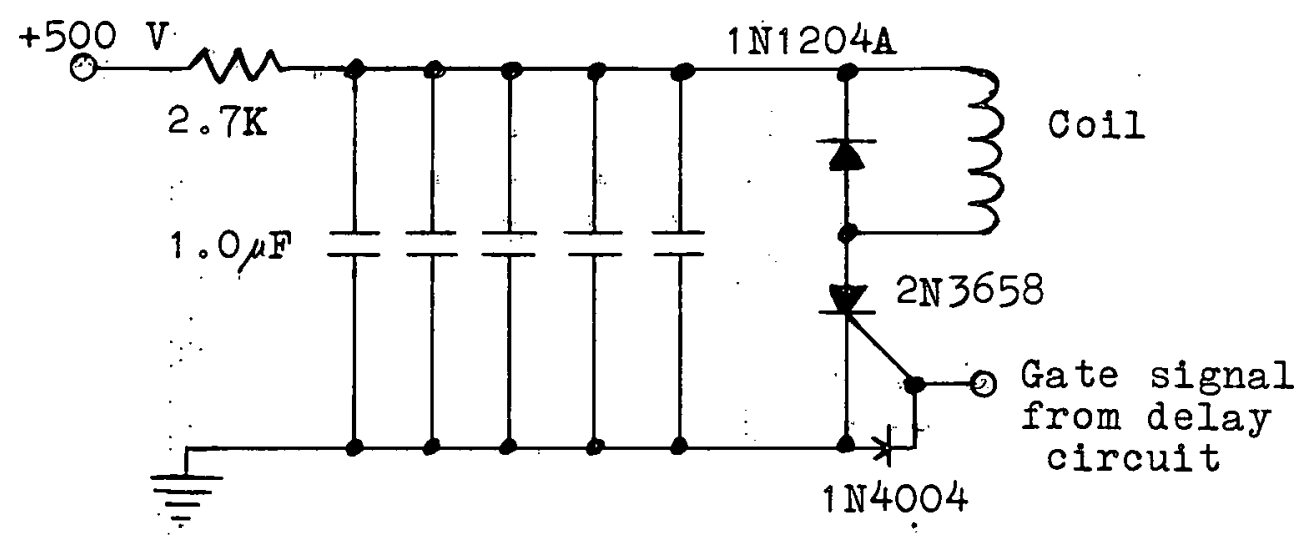




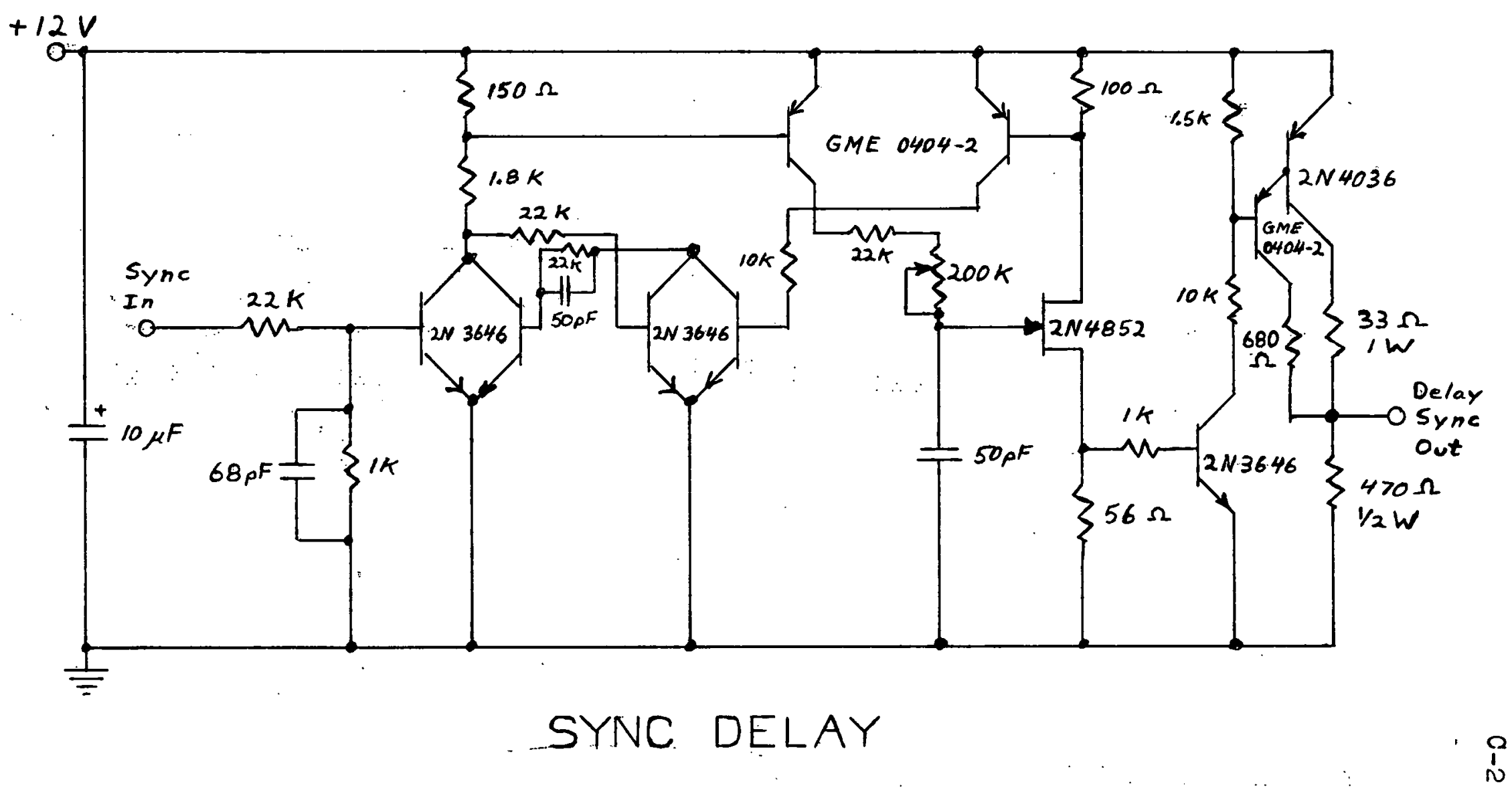


Specific Heat Apparatus

The 1mportant features of the specific heat apparatus are shown in the diagram on page $D-4$. We were interested in setting up the experiment rapidly for rough measurements. Therefore, the apparatus lacks many of the refinements commonly employed in such measurements. With this crude design, data accurate to $1 \%$ were obtained.

The sample was cut from a single crystal of $\mathrm{Mn}_{2-\mathrm{x}_{\mathrm{x}} \mathrm{Cr}} \mathrm{Sb}$. The chromium concentration was about $\mathrm{x}=0.033$, so we expected reorientation to occur at $215^{\circ} \mathrm{K}$. The sample weighed $2.55 \mathrm{~g}$ and had dimensions of $0.2 \mathrm{~cm} \mathrm{X}$ $1.9 \mathrm{~cm} \mathrm{X} 1.6 \mathrm{~cm}$.

The brass can was immersed in a liquid nitrogen bath and was pumped down to a pressure of $10^{-6} \mathrm{mmHg}$. The sample was suspended in the middle of the can by cotton thread. The electrical leads were one foot of \#44 copper wire colled around the thread. These precautions for thermal isolation were superfluous since the thermal flux due to radiation was later found to be 10 times the thermal conduction down the wire.

Two heaters were used. The $500 \Omega$ power heater consisted of 10 ft of manganin wire wrapped on the two ends of the sample. On top of this was wound the $200 \Omega$ holding heater with 50 ft of manganin wire. None 
of these wires touched the thermocouple which was placed on the surface in the middle of the sample. Everything was cemented to the sample w1th G.E. \#7031 adhesive and insulating varnish which has good thermal conductivity. The total weight of the wire and varnish was $0.28 \mathrm{~g}$. Thermal equilibrium throughout the sample was achieved in about 15 seconds.

Specific heat measurements were made by applying $1 / 10 \mathrm{~W}$ for $15 \mathrm{sec}$ to the sample and measuring the change in temperature which was about $1 / 2-3 / 4$ of a degree. The thermocouple voltage was monitored on a chart.recorder. The power applied to the holding heater was gradually increased so that sample was always within two degrees of its equilibrium temperature.

We used a H-P 6lola power supply which was stable to better than $0.01 \%$. The time intervals were measured to \pm 0.01 sec or about $\pm 0.01 \%$. The timing clock and power supply were activated by a single switch.

The major source of error was the temperature measurement. The thermocouple reference function was fixed to a copper block in the bottom of the liquid nitrogen bath. From the chart recorder the changes in temperature were measured with an accuracy of about 1\%. This accounts for the magnitude of the scatter In our data. 
of these wires touched the thermocouple which was placed on the surface in the middle of the sample. Everything was cemented to the sample wIth G.E. \#7031 adhesive and insulating varnish which has good thermal conductivity. The total welght of the wire and varnish was $0.28 \mathrm{~B}$. Thermal equilibrium throughout the sample was achleved in about 15 seconds.

Specific heat measurements were made by applying 1/10 W for $15 \mathrm{sec}$ to the sample and measuring the change in temperature which was about $1 / 2-3 / 4$ of a degree. The thermocouple voltage was monitored on a chart.recorder. The power applied to the holding heater was gradually increased so that sample was always within two degrees of its equilibrium temperature.

We used a H-P 6101A power supply which was stable to better than $0.01 \%$. The time intervals were measured to \pm 0.01 sec or about $\pm 0.01 \%$. The timing clock and power supply were activated by a single switch.

The major source of error was the temperature measurement. The thermocouple reference junction was fixed to a copper block in the bottom of the liquid nitrogen bath. From the chart recorder the changes in temperature were measured with an accuracy of about 1\%. This accounts for the magnitude of the scatter in our data. 
Our results show no specific heat anomalies in the temperature range between $200^{\circ}$ and $240^{\circ} \mathrm{K}$. The $2 \%$ anomaly should have fust been visible. We did not pursue the measurement any further. To be worthwhile the sensitivity of the apparatus would be to be $1 \mathrm{~m}-$ proved by at least a factor of 10 and this would become a signtficant research project requiring a considerable amount of time. 


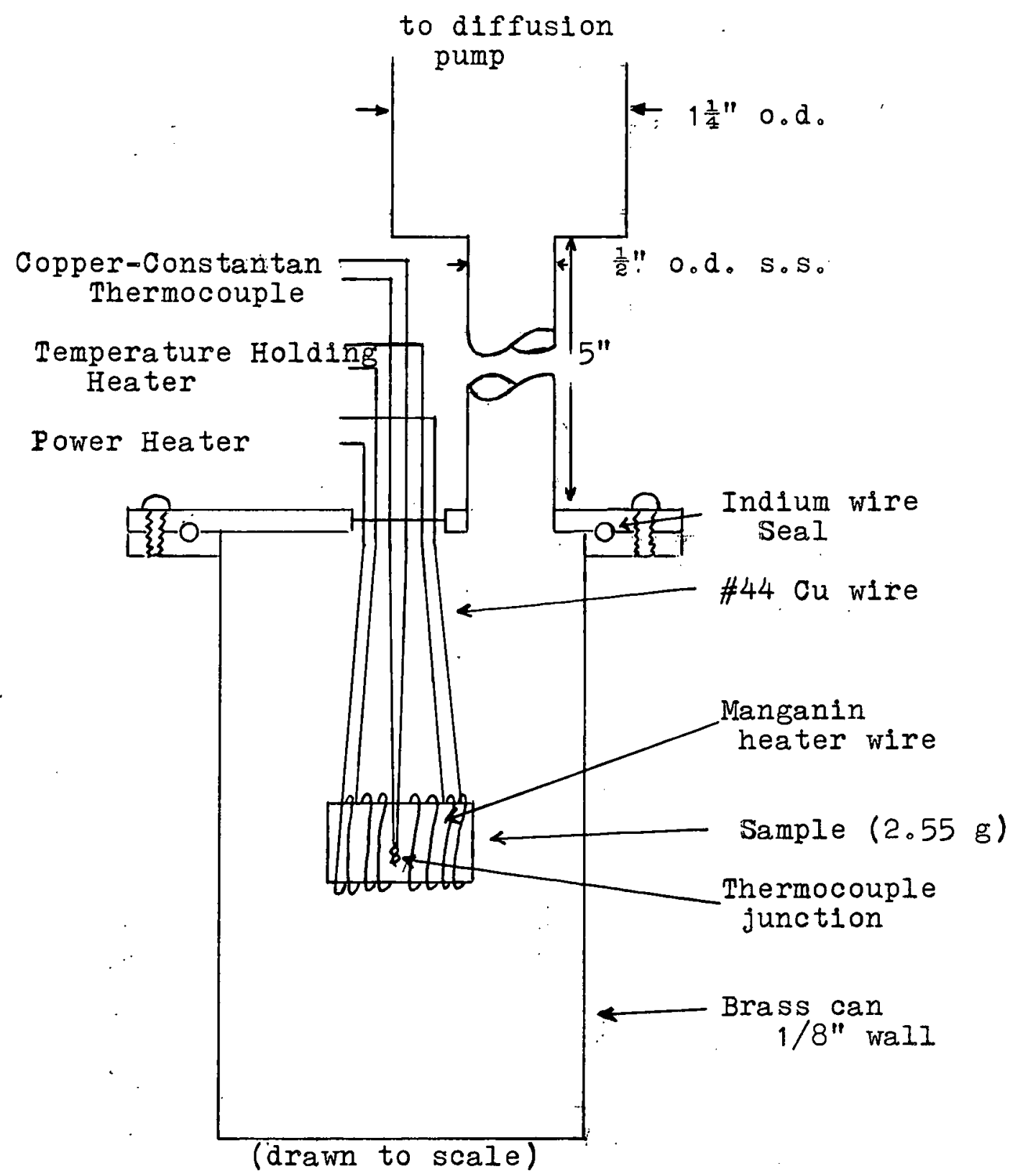

SPECIFIC HEAT APPARATUS 


\section{References}

1. For a review of the fundamentals of nuclear magnetic resonance see:

a. C.P.Slichter, Principles of Magnetic

Resonance with Examples from Solid State Physics (Harper and Row, New York, 1963).

b. A. Abragam, The Principles of Nuclear Magnetism (oxford University Press, Iondon, 1961).

2. M. A. Grace, C. E。 Johnsön, NoKurti, R。G。 Scurlock, and R. To Taylor, Commun, Conf. Basses Temperature, Paris, 1955, p。263 (1955); and Phil. Mag。 [8] 4, 948 (1959)。

3. A. C。 Gossard and A. Mo Port1s, Phys。 Rev。 Letters 3. 164 (1959).

4. R.C. LeCraw, R.Wolfe, E。M。Gyorgy, R。 B。 Hagedorn, J。C.Hensel, and $J$ 。 P. Remeika, J.Appl。Phys。 39, 1019 (1968).

5. Ho Horner and C.M. Varma, Phys。Rev。 Letters 20, 845 (1968)。

6. C. Guillaud, Thesis Strasbourg (1943).

7. M。 Ko Wilkinson, No S. Gingrich, and C。G。 Shull, J. Phys. Chem. Solids 2, 189 (1957).

8. H.A. Alperin, P. J。 Brown, and R. Nathans, J.Appl. Phys. 24, 1201:(1963)。

9. R. E. Watson and A. JoFreeman, Acta Cryst. 14, $27(1961)$ 。

10. J. B. Goodenough, Magnetism and the Chemical Bond (John Wiley and Sons,.New York, 1966), p.291.

11. T. Jo Swoboda, W. H. Cloud, T. A. Bitter, M. S。 Sadler, and H. S. Jarrett, Phys。Rev. Letters 4 , $509(1960)$.

12. W. H. Cloud, H. S. Jarrett, A. E. Austin, and E. Adelson, Phys. Rev. 120, $1969^{\circ}(1960)$. 
13. F. J. Darnell, W。 Ho Cloud, and H. S. Jarrett, Phys. Rev.130, 647 (1963).

14. So Abe, J. Phys。 Soc. Japan 21, 551 (1966).

15. P.E. Bierstedt, Phys。Rev。 132, 609 (1963)。

16. Co Kittel, "Phys. Rev。120, 335 (1960).

17. P. Eo, Bierstedt, F。 Jo Darnell, W. Ho Cloud, R。 B。 Flippen, and $H_{0}$ S. Jarrett, Phys。Rev。 Letters $\underline{8}, 15$ (1962).

18. I. Néel, Ann. Phys. (N.Y.) 3, 137 (1948).

19. Takasht Tonegaw, J. Phys。 Soc。 Japan 17, 1398 (1962)。

20. J.D.Wolf and J。E. Hanlon, J.Appl. Phys 32, 2584 (1961).

21. C. Kittel and J.K. Galt, Solid state Physics, edited by F.Seitz and D. Turnbull (Academic Press, New York), Vol。3, 450 (1956)。

22. E. Fermi, Z。Physik 60, 320 (1930).

23. E. Fermi and E. Segrè, Rend.Accad. Nazl。 Inincel 4, 18 (1933); and Z. Physik 82, 729 (1933)。

24. A. J. Freeman and R. E。 Watson, in Magnetism, edited by G。T. Rado and H. Suhl (Academic Press, Inc。, New York, 1955), Vol。II(A)。

25. R. E. Watson and A. Jo. Freeman, in Hyperfine Interactions, edited by $A_{0} J$ 。 Freeman and $R_{0} B$. Frankel (Academic Press, New York, 1967):

26. R:M. Sternheimer and H०M. Foley, Phys. Rev。 22, 1460 (1953);

R. M. Sternheimer, Phys. Rev。 80, 102 (1950).

27. A. Jo Freeman and R. E。 Watson, Phys. Rev。 123, 2027 (1961).

28. A. Abragam, JoHorowitz, and M. Ho I. Pryce, Proc。Roy。Soc。(Iondon) A230, 169 (.1955)。

29. R. Winkler, Phys. Letters 23, 301 (1966). 
30. M. A. Ruderman and C. Kittel, Phys.Rev. 96,

99 (1954);

T. Kasuya, Progr. Theoret. Phys。(Kyota) 16,

45 (1959);

K. Yosida, Phys. Rev. 106, 893 (1957).

31. A. M. Portis and A. C. Gossard, J.Appl. Phys. 31. 205S (1960).

32. A。M. Portis and R.H. Lindquist, in Magnetism, edited by $G$. Rado and H. Suhl (Academic Press, New York, 1965), Vol。II(A), p. 358.

33. E。 I. Hahn, Phys, Rev。 80, 580 (1950).

34. For a description of this procedure and its applications see:

I. I。 Rabi, N。 F。 Ramsey and J. Schwinger, Rev。Mod。Phys。 26, 167 (1954).

35. E. I. Hahn and D。E。 Maxwell, Phys. Rev. 88, 1.070 (1955);

M. Bloom, Phys。Rev。97, 1699 (1955)。

36. H. Abe, H。 Yasuoka, and A。 Hira1, J。 Phys。Soc。 Japan 21, 77 (1966)。

37. For the general forms of these operators see:

$$
\begin{aligned}
& \text { M. E. Rose, Elementary Theory of Angular } \\
& \text { Momentum (John Wiley and Sons, Inco, } \\
& \text { New York, 1957). }
\end{aligned}
$$

38. K. W。 H。 Stevens, in Magnetism, edited by G。T。 Rado and H. Suhl (Academic Press, New York, 1963), Vol。I, p. 5.

39. P. G。 deGennes, P. Pincus, F。Hartman-Boutron, and J。M.Winter, Phys, Rev。129, 1105 (1963).

40. A. JoHeeger and T. W. Houston, Phys. Rev. 135, A661 (1964)。

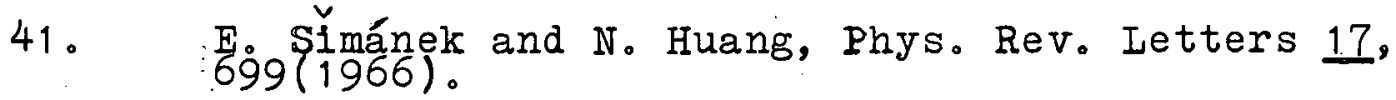

42. Wo MoWalsh, Jr。, Jean Jeener, and No Bloembergen, Phys. Rev。139, A1338 (1965).

43. E. Símánek and R。Orback, Phys。Rev。 145, 191 $(1966)$. 
44. Chao-Yuan Huang, Physics 3, 231 (1967).

45. J。M.Winter, Phys。Rev。124, 452 (1961).

46. A. Narath, Phys。Rev, 140, A854 (1965).

47. Ho Yasuoka, V。 Jaccarino, and R。To Lewis, Bull. Am。 Phys。Soc。13, 473 (1968).

48: H. Suhl, Phys Rev. 109, 606 (1958); 542 (1958).

49. Mo Weger, E。 I. Hahn, and A. M. Portis, J.Appl. Phys. 32, 124S (1961)。

50. M. Weger, Phys. Rev. 128, 1505 (196?).

51. Tôru Moriya, J。 Phys. Soc。 Japan 19, 681 (1964).

52. R. E. Walstedt, V. Jaccarino, and N. Kaplan, J.Phys.Soc.Japan 21, 1843 (1966).

53. M. Hansen, Constitution of Binary Alloys (McGraw-Hili Book Co., Inc., New York, 1958), p. 951 。

54. These temperatures were reported by H. S. Jarrett (private communication).

55. We thank H. S. Jarrett of the Central Research Department Experimental Station, E. I. duPont de Nemours and Co., Wilmington, Delaware for providing us with various samples of $\mathrm{Mn}_{2-x} \mathrm{Cr}_{\mathrm{x}} \mathrm{Sb}$.

56. S. Foner, Rev. Sci. Inst. 30, 548 (1959).

57. M. B. Sterns, Phys。Rev。 162, 496 (1968).

58. To Hihara, $Y_{0}$ Ko1, and A. Tsujimura, J. Phys. Soc.Japan 17, 1320 (1962).

59. D. H. Martin, Magnetism in Solids (MIT Press, Cambridge, Mass。, 1967), p。398.

60. Ibid. p. 425.

61. N. Kaplan, V. Jaccarino, and JoH。 Wernick, Phys。Rev。 Jetters 16, 1142 (1966)。 


\section{$R-5$}

62. R. W. Houghton and W. Weyhman, Phys. Rev. Letters 20, $842(1968)$.

63. H. S. Jarrett, P.E. Bierstedt, F。J.Darnell, and M. Sparks, J. Appl。Phys。 32, 575 (1961)。

64. J. R. Shane, Phys. Rev. Letters 20, 728 (1968).

65. M. Bloom, E. I. Hahn, and B. Herzog, Phys。 Rev。 97, 1699 (1955). 